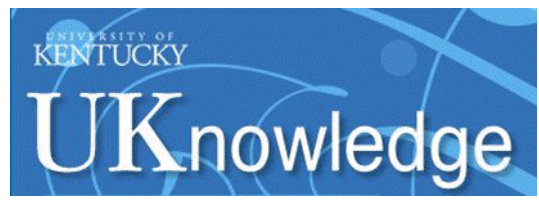

University of Kentucky

UKnowledge

\title{
Adverse Events and Treatment Discontinuations of Antimuscarinics for the Treatment of Overactive Bladder in Older Adults: A Systematic Review and Meta-Analysis
}

\author{
Scott Martin Vouri \\ St. Louis College of Pharmacy \\ Clark D. Kebodeaux \\ University of Kentucky, clark.kebodeaux@uky.edu \\ Paul M. Stranges \\ Univeristy of Illinois at Chicago \\ Besu F. Teshome \\ St. Louis College of Pharmacy
}

Follow this and additional works at: https://uknowledge.uky.edu/pps_facpub

Part of the Geriatrics Commons, Gerontology Commons, and the Pharmacy and Pharmaceutical Sciences Commons

Right click to open a feedback form in a new tab to let us know how this document benefits you.

\author{
Repository Citation \\ Vouri, Scott Martin; Kebodeaux, Clark D.; Stranges, Paul M.; and Teshome, Besu F., "Adverse Events and \\ Treatment Discontinuations of Antimuscarinics for the Treatment of Overactive Bladder in Older Adults: A \\ Systematic Review and Meta-Analysis" (2017). Pharmacy Practice and Science Faculty Publications. 41. \\ https://uknowledge.uky.edu/pps_facpub/41
}

This Review is brought to you for free and open access by the Pharmacy Practice and Science at UKnowledge. It has been accepted for inclusion in Pharmacy Practice and Science Faculty Publications by an authorized administrator of UKnowledge. For more information, please contact UKnowledge@lsv.uky.edu. 
Adverse Events and Treatment Discontinuations of Antimuscarinics for the Treatment of Overactive Bladder in Older Adults: A Systematic Review and MetaAnalysis

Digital Object Identifier (DOI)

https://doi.org/10.1016/j.archger.2016.11.006

Notes/Citation Information

Published in Archives of Gerontology and Geriatrics, v. 69, p, 77-96.

(c) 2016 Elsevier Ireland Ltd.

This manuscript version is made available under the CC-BY-NC-ND 4.0 license http://creativecommons.org/licenses/by-nc-nd/4.0/ 


\title{
Adverse events and treatment discontinuations of antimuscarinics for the treatment of overactive bladder in older adults: a systematic review and meta-analysis
}

\author{
Scott Martin Vouri, PharmD, MSCI ${ }^{1}$, Clark D. Kebodeaux, PharmD ${ }^{2}$, Paul M. Stranges, \\ PharmD $^{3}$, and Besu F. Teshome, PharmD, MSc ${ }^{1}$ \\ ${ }^{1}$ St. Louis College of Pharmacy, 4588 Parkview Place, St. Louis, Missouri, United States \\ 2University of Kentucky - College of Pharmacy, Lexington, Kentucky, United States 789 S. \\ Limestone, Lexington, Kentucky, United States \\ 3Univeristy of Illinois at Chicago - College of Pharmacy, Chicago, Illinois, United States 833 \\ South Wood Street, Chicago, Illinois, United States
}

\begin{abstract}
Introduction-Antimuscarinics should be used with caution in older adults with overactive bladder (OAB) due to anticholinergic adverse events (AEs). Systematic reviews and meta-analyses (SRMAs) have analyzed safety-related outcomes but have not specified risk in the elderly, the population at highest risk for AEs. The aim of this review is to explore and evaluate AEs and treatment discontinuations in adults 65 or older taking antimuscarinics for OAB.

Methods-Keywords were searched in MEDLINE, EMBASE, SCOPUS, and Cochrane Central Register for Controlled Trials. Randomized controlled trials (RCTs) along with sub-analyses and pooled analyses that compared antimuscarinics to placebo or another antimuscarinic were performed in February 2015. Studies assessing AEs or treatment discontinuations in a population of adults 65 or older were included. The Jadad Criteria and McHarm Tool were used to assess the quality of the trials.
\end{abstract}

Results-A total of 16 studies met the inclusion criteria. Eighty AEs and 27 reasons for treatment discontinuation were described in the included studies and further explored. Anticholinergic AEs were more common in antimuscarinics compared to placebo. Incidence of dizziness, dyspepsia, and urinary retention with fesoterodine, headache with darifenacin, and urinary tract infections with solifenacin were significantly higher compared to placebo. Treatment discontinuation due to AEs and dry mouth were higher in the antimuscarinics when compared to placebo in older adults.

Corresponding Author: Scott Martin Vouri, PharmD, MSCI, Address: 4588 Parkview Place, St. Louis, Missouri, 63110 Telephone: 1-314-446-8551 Fax: 1-314-446-8500 scott.vouri@ stlcop.edu.

Publisher's Disclaimer: This is a PDF file of an unedited manuscript that has been accepted for publication. As a service to our customers we are providing this early version of the manuscript. The manuscript will undergo copyediting, typesetting, and review of the resulting proof before it is published in its final citable form. Please note that during the production process errors may be discovered which could affect the content, and all legal disclaimers that apply to the journal pertain. 
Conclusions-Treatment for overactive bladder using antimuscarinics in adults aged 65 or older resulted in significant increases in risk for several AEs compared to placebo including anticholinergic and non-anticholinergic AEs.

\section{Keywords}

Adverse events; Antimuscarinics; Elderly; Meta-analysis; Overactive bladder; Systematic review

\section{Introduction}

Overactive bladder $(\mathrm{OAB})$ is a condition that can negatively impact quality of life in older adults. The prevalence of OAB in epidemiological studies increases with age (Gomelsky, 2009; Milsom et al., 2000; Tubaro, 2004). Signs and symptoms of OAB such as urinary frequency, urgency, nocturia, and incontinence affect $25 \%$ of adults aged 60 or older (Scheife and Takeda, 2005; Wagg et al., 2010). There are several oral and non-oral treatment options for patients with $\mathrm{OAB}$ which include antimuscarinics (oxybutynin, tolterodine, trospium, darifenacin, solifenacin, fesoterodine) and a beta-3 agonist (mirabegron) (Kraus et al., 2010a; Macdiarmid, 2008). These medications are all viable options for the elderly. owever, providers should be cautioned in using these medications due to adverse drug events (AEs) including dry mouth, blurry vision, and constipation in antimuscarinics as well as hypertension in beta-3 agonists (Sternberg et al., 2011). These AEs may contribute to nonadherence or discontinuation of the prescribed medication, which can lead to a return of OAB symptoms and a reduction in quality of life (Benner et al., 2010).

Several systematic reviews and meta-analyses have evaluated the use of medications in the treatment of OAB (Chapple et al., 2015; Chapple et al., 2008; Cui et al., 2014; Herbison et al., 2003; Huang et al., 2015; Kessler et al., 2011; Luo et al., 2012; Madhuvrata et al., 2012; Novara et al., 2008; Paquette et al., 2011; Reynolds et al., 2015; Roxburgh et al., 2007; Wu et al., 2014; Wyndaele et al., 2014). These reviews compared OAB medications to placebo and/or to other $\mathrm{OAB}$ medications, either directly or indirectly, using prospective randomized, non-randomized, and retrospective observational trials. Current systematic reviews have only described the individual studies with regards to the use of OAB medications in the elderly. No systematic review has used a meta-analytical technique to broadly explore safety outcomes in the elderly. The goal of this systematic review is to perform exploratory analyses of AEs and treatment discontinuations in oral and non-oral medications used to treat $\mathrm{OAB}$ in studies with patients aged 65 or older.

\section{Materials and Methods}

The Preferred Reporting Items for Systematic Reviews and Meta-Analysis (PRISMA) statement was used for reporting this review. Institutional review board approval was not required for this review.

\subsection{Literature Search}

Studies involving patients aged 65 or older with $\mathrm{OAB}$ or urge urinary incontinence who received either an antimuscarinic (oxybutynin, tolterodine, trospium, solifenacin, 
darifenacin, fesoterodine) or a beta-3 agonist (mirabegron) were included. Randomizedcontrolled trials (RCTs) of one agent versus another agent or placebo (including studies with ztwo treatment arms), sub-analysis of a parent RCT, or pooled-results of two or more parent RCTs were included (Paquette et al., 2011). A parent study was defined as the original publication(s) in which a sub-analysis or pooled analysis was derived. Studies less than 4 weeks in duration, those evaluating antimuscarinics in combination with alpha-blockers for lower urinary tract symptoms, populations in which neurogenic bladder or conditions other than $\mathrm{OAB}$ were studied, and any language other than English were excluded by title or abstract.

The following electronic databases were searched: MEDLINE (PubMed interface), EMBASE, SCOPUS, and Cochrane Central Register for Controlled Trials. The basic search strategy used derivations of the following strategy (aged AND [antimuscarinic agents OR beta-3 agonists $\mathrm{OR}$ oxybutynin OR tolterodine OR trospium OR darifenacin OR solifenacin OR fesoterodine OR mirabegron]). MeSH and EMTREE terms were used for MEDLINE and EMBASE, respectively, and the full search strategy is described in Appendix 1.

Duplicates were removed and non-relevant studies were removed based on title and abstract (SMV); relevant full-text articles were identified. Two of the four authors (SMV, PMS, CDK, BFT) independently screened each full-text article using the pre-established criteria after a team training exercise. Studies were excluded for the following reasons: 1) did not report original data, 2) did not include an overactive bladder medication, 3) no comparator arm, 4) used a non-standard dose, route, and frequency (e.g., antimuscarinic combination therapy), 5) conducted in subjects without overactive bladder, 6) assessed only quality of life outcomes, 7) trial was less than 4 weeks in duration, 8) was not a randomized, controlled trial, 9) studies with outcomes that did not differentiate by age, 10) did not report safety outcomes, and 11) used a duplicate population. Any conflicts were resolved by consensus of the two authors.

\subsection{Data Extraction}

As this was an exploratory analysis, all AEs and treatment discontinuations reported in any included study were identified and collected by two authors (SMV, CDK) after a team training exercise. Any conflicts were resolved by consensus of the two authors. Once confirmed, a data extraction sheet was created. Study characteristics were extracted which included the last name of the first author, year published, journal, study type, study duration, overactive bladder medication and comparator, number of subjects, age cut points, OAB inclusion criteria, percent female, location of study, AEs, and treatment discontinuations as previously defined. Authors were contacted by email when all necessary data was not available in the manuscript; no additional data was incorporated from authors contacted by email. Two of four authors (SMV, PMS, CDK, BFT) independently extracted each study using the pre-established criteria after an additional training exercise. Any conflicts were resolved by consensus of the two authors. As AEs and treatment discontinuations were reported as dichotomous variables within the study, the timing of such events could not be assessed. 


\subsection{Quality Assessment}

The Jadad Criteria was used to appraise the risk of bias within the included studies (Jadad et al., 1996). For pooled analyses and sub-analyses studies, the parent studies were scored when available. The McMaster tool for assessing quality of harms assessment and reporting in study reports (McHarm Tool) was used to examine the consistency in reporting of AEs (Santaguida and Raina, 2008). Each study was extracted independently by two of four authors (SMV, PMS, CDK, BFT) using the pre-established criteria to create the Jadad Criteria Score and complete the McHarm Tool after an additional training exercise. A study with a Jadad Criteria score of 4 or greater was considered high quality. Any conflicts were resolved by consensus of the two authors.

\subsection{Data Synthesis}

The studies were synthesized using the above criteria and included the risk of bias and quality. These studies were assessed for heterogeneity using the $\mathrm{I}^{2}$ statistic; as a conservative estimate, random-effects models were used for all analyses. If the $\mathrm{I}^{2}$ statistic is $<25 \%$, then the authors reported no heterogeneity. If the $\mathrm{I}^{2}$ statistic is $225 \%$ then it will be denoted in the tables and the value reported out in the manuscript. Given that only four studies with four separate medications reported outcomes by differing doses, doses within each medication category were collapsed (Foote et al., 2005; Kraus et al., 2010b; Malone-Lee et al., 2001; Wagg et al., 2006). Medications were compared to placebo and any active comparator in separate analyses. Individual AEs and treatment discontinuations were described in rate ratios in an overall analysis and an analysis stratified by specific medication. For statistically significant AEs and treatment discontinuations, number needed to harm $(\mathrm{NNH})$ were also calculated.

The lack of standardization in the reporting of AEs across studies was accounted for by combining similar AEs extracted from an individual study based on symptomatology to improve power in secondary analyses similar to the Common Terminology Criteria for Adverse Events v3.0 (CTCAE) (2009; Kessler et al., 2011).

\section{Results}

The search retrieved a total of 26,166 studies, of which 228 full-text studies were reviewed for inclusion in the systematic review (Figure 1). The most common reasons for exclusion were outcomes not being differentiated by age or a lack of a comparator arm. Seventeen studies (7 RCTs, 3 sub-analyses of RCTs, and 7 pooled analyses of RCTs) made the inclusion criteria in which AEs were reported in the 65 or older population. Safety endpoints could not be extracted from one pooled analysis of RCTs (Griebling et al., 2009) as AEs were reported graphically; therefore, 16 studies were used in these analyses (Chapple et al., 2007a; Dubeau et al., 2014; DuBeau et al., 2012; Foote et al., 2005; Herschorn et al., 2011; Kraus et al., 2010b; Lackner et al., 2008; Malone-Lee et al., 2001; Minassian et al., 2007; Sand et al., 2011; Sand et al., 2004; Szonyi et al., 1995; Wagg et al., 2014; Wagg et al., 2013; Wagg et al., 2006; Zinner et al., 2002). 
Study characteristics and Jadad Criteria Score (Table 1) and McHarm Tool (Appendix 2) were calculated from the included studies. The parent RCTs of the sub-analyses and pooled analyses, when available, were extracted to assess study duration, OAB inclusion criteria, location of study, Jadad Criteria Score (Table 1), and McHarm Tool (Appendix 2) (Appell et al., 2001; Cardozo et al., 2004; Chapple et al., 2007b; Chapple et al., 2004a; Chapple et al., 2005; Chapple et al., 2004b; Dmochowski et al., 2008; Herschorn et al., 2013; Herschorn et al., 2010a; Herschorn et al., 2010b; Kaplan et al., 2011; Khullar et al., 2013; Nitti et al., 2013; Nitti et al., 2007; Staskin et al., 2007; Van Kerrebroeck et al., 2001). The parent studies ( 3 studies) from one pooled analysis were unable to be assessed by the authors as the results were unpublished (Foote et al., 2005).

\subsection{Study Quality}

Five of the 7 RCTs studies and 10 of the 16 parent studies evaluated were of high quality (Table 1). The quality of AEs reporting in the RCTs and the parent studies reporting was assessed using the McHarm Tool (Appendix 2). Specifically, question 11 of the McHarm Tool asked "Did the authors specify if the harms reported encompass ALL the events collected, or a selected SAMPLE?" The authors answered either no or unclear to this question in all evaluated studies. Of the 23 studies that were evaluated (7 RCTs and 16 parent studies) for reporting all events, 14 studies defined their parameters for reporting specific AEs (i.e., only if $\geq 2 \%$ in any group). It was unclear what proportion of AEs were reported in the remaining 9 studies.

\subsection{Overactive Bladder Medications}

The 16 included studies included analyses on oxybutynin immediate-release (IR) (Herschorn et al., 2011; Minassian et al., 2007; Szonyi et al., 1995), oxybutynin extended-release (ER) (Lackner et al., 2008; Minassian et al., 2007; Sand et al., 2011; Sand et al., 2004), tolterodine IR (Malone-Lee et al., 2001; Sand et al., 2004), tolterodine ER (DuBeau et al., 2012; Wagg et al., 2014; Zinner et al., 2002), trospium ER (Sand et al., 2011), solifenacin (Herschorn et al., 2011; Wagg et al., 2006), darifenacin (Chapple et al., 2007a; Foote et al., 2005), fesoterodine (Dubeau et al., 2014; DuBeau et al., 2012; Kraus et al., 2010b; Wagg et al., 2013), and mirabegron (Wagg et al., 2014). Thirteen studies were placebo controlled. Two studies compared solifenacin and oxybutynin ER to oxybutynin IR (Herschorn et al., 2011; Minassian et al., 2007), one study compared oxybutynin ER to tolterodine IR (Sand et al., 2004), and one study compared fesoterodine to tolterodine ER (DuBeau et al., 2012). One study compared tolterodine ER, fesoterodine, mirabegron, and placebo (Wagg et al., 2014). Using this study, the authors compared tolterodine ER to fesoterodine, fesoterodine and tolterodine ER to the same cohort of placebo. Additionally, this was the only study to assess mirabegron (compared to placebo and compared to tolterodine ER) (Wagg et al., 2014). Due to the differing mechanism of action and AEs relative to antimuscarinics, mirabegron was not incorporated into the meta-analysis as the differences in AEs and treatment discontinuations were already reported.

In these analyses, 4,188 subjects (3952 subjects in placebo-controlled trials) aged 65 or older were randomized to antimuscarinics medications for 4 to 12 weeks and 3,026 were randomized to placebo. The number of subjects randomized to antimuscarinic treatment was 
oxybutynin IR ( $\mathrm{n}=88)$, oxybutynin ER ( $\mathrm{n}=115)$, tolterodine IR $(\mathrm{n}=194)$, tolterodine ER $(\mathrm{n}=992)$, trospium ER $(\mathrm{n}=85)$, solifenacin $(\mathrm{n}=650)$, darifenacin $(\mathrm{n}=650)$, fesoterodine $(\mathrm{n}=1591)$.

From the overall 16 studies included for analyses, there were 80 distinct reports of AEs and 27 distinct reports of treatment discontinuation. Many AEs reported severity of disease (i.e., mild to moderate urinary tract infection and severe urinary tract infection) without defining severity levels. As standard nomenclature was not routinely used, many AEs appeared to be very similar (i.e., vision disorder and blurry vision) which likely increased the number that were explored.

Outcomes for all AEs and treatment discontinuations for antimuscarinics versus placebo (Appendices 3,5) and antimuscarinic versus antimuscarinic (Appendices 4,6) are described regardless of being statistically significant. There were 14 statistically significant AEs (Table 2), two statistically significant treatment discontinuations (Table 4), and one statistically significant combined AEs (Appendix 7) in the antimuscarinic versus placebo analyses. There were four statistically significant AEs (Table 3), one statistically significant treatment discontinuation (Table 5), and no statically significant combined AEs (Appendix 8) in the antimuscarinic versus antimuscarinic analyses.

\subsection{Adverse Events}

3.3.1 Antimuscarinics versus Placebo-Statistically significant rates for AEs and NNH for antimuscarinics compared to placebo are described in Table 2. The proportion of subjects with any AEs was $53.8 \%$ in those receiving an antimuscarinic compared to $41.7 \%$ in subjects receiving placebo. There was also an increase in treatment-related AEs which was more narrowly defined as adverse outcomes likely related to the study drug in studies assessing darifenacin and trospium ER.

Ten percent of subjects receiving an antimuscarinic experienced constipation compared to $3.3 \%$ receiving placebo $\left[\mathrm{I}^{2}=46.1 \%\right]$. Darifenacin $(18.0 \%)$, solifenacin $(15.4 \%)$, fesoterodine $(8.5 \%)$, and tolterodine $(4.8 \%)$ had significantly higher rates of constipation among subjects receiving antimuscarinics compared to placebo. Within the same studies, $25 \%$ of subjects receiving an antimuscarinic experienced dry mouth compared to $5.3 \%$ of subjects receiving placebo $\left[I^{2}=73.9 \%\right]$. There was a significantly higher rate of dry mouth with no heterogeneity among subjects in tolterodine ER (6.1\%), darifenacin (23.8\%), solifenacin (26.0\%), and fesoterodine (29.4\%) groups. A significantly higher proportions of subjects receiving an antimuscarinic experienced mild, mild-moderate, moderate, and severe dry mouth when compared to placebo.

There was no overall increase rate of dizziness in antimuscarinics compared to placebo; however, in three fesoterodine studies there was over a higher rate of dizziness (2.5\%) compared to placebo $(1 \%)$. Subjects receiving fesoterodine had significantly higher rate of dyspepsia (2.7\%) compared to placebo ( $0.6 \%)$. In a subsequent analysis in which all gastrointestinal AEs were combined (abdominal pain, anorexia, dyspepsia, gastrointestinal AEs, gastroesophageal reflux disorder, heartburn), there was no significant difference in antimuscarinics (4.6\%) compared to placebo (2.7\%); however, in the three fesoterodine 
studies that assessed any of these AEs, there was a significantly higher rate of gastrointestinal AEs (3.1\%) compared to placebo (1.1\%) (Appendix 7). There was no overall difference in headache between subjects receiving antimuscarinic compared to placebo; however, a higher rate was seen in the three studies in which subjects received tolterodine ER $(1.7 \%)$ compared to placebo $(0.7 \%)$. There was an overall higher rate of urinary retention in subjects receiving antimuscarinics $(1.3 \%)$ compared to placebo $(0.4 \%)$ and a higher rate in the four studies which assessed subjects receiving fesoterodine (1.7\%) compared to placebo $(0.2 \%)$. There was an increased rate of urinary tract infections in one study comparing solifenacin (6.0\%) to placebo (3.1\%); however, there was no difference in antimuscarinics (3.7\%) overall versus placebo (2.8\%).

3.3.2 Head-to-Head Trials-Statistically significant rate of AEs and NNH for an antimuscarinic compared to another antimuscarinic are described in Table 3. In these comparator studies, solifenacin exhibited a lower rate of AEs (70.4\% versus 96.7\%), treatment-related AEs (60.3\% versus $86.7 \%$ ), and dry mouth (37.0\% versus $80.0 \%$ ) compared to oxybutynin IR, respectively. Subjects receiving fesoterodine were more likely to experience AEs (54.8\% versus $40.0 \%$ ) and dry mouth (31.3\% versus $14.7 \%$ ) compared to tolterodine ER. There were no significant AEs when outcomes were combined based on symptomatology (Appendix 7).

\subsection{Treatment Discontinuations}

3.4.1 Antimuscarinic versus Placebo-Statistically significant rate of treatment discontinuation and NNH for antimuscarinics compared to placebo are described in Table 4 . Of the 27 reasons for discontinuation, only two were statistically significant. Treatment discontinuation due to AEs was higher in the antimuscarinic group (7.1\%) compared to placebo (5.0\%); however, when stratified by medication, only fesoterodine (9.4\%) compared to placebo (4.9\%) was statistically significant for treatment discontinuation due to an $\mathrm{AE}$. Overall, the discontinuation rate due to dry mouth was higher in antimuscarinics $(1.5 \%)$ compared to placebo $(0.4 \%)$ and in three comparisons of fesoterodine $(2.0 \%)$ when compared to placebo $(0.3 \%)$.

3.4.2 Head-to-Head Trials-Statistically significant rate of treatment discontinuation and $\mathrm{NNH}$ for an antimuscarinic compared to another antimuscarinic are described in Table 4. In the antimuscarinic comparators, only fesoterodine (8.6\%) had a higher rate of discontinuation due to AEs compared to tolterodine ER (2.7\%).

\section{Discussion}

The aim of these analyses was to assess the rate of AEs and treatment discontinuations with the use of antimuscarinics in the group of patients who are most likely to experience OAB. With a known lack of studies specific to older adults, sub-analyses and pooled analyses were included to increase the number of subjects similar to a previous systematic review and meta-analysis of antimuscarinics in older adults (Paquette et al., 2011). Data from the parent studies were used to assess for OAB inclusion criteria, location of study, and quality of studies. There were 18 comparisons from 16 studies which assessed several antimuscarinic 
agents used for the treatment of $\mathrm{OAB}$. A majority of studies were placebo-controlled while only 4 studies included direct comparisons to two antimuscarinic agents. In general, these studies were very similar in the included age (as only two studies used a minimum age inclusion criterion older than 65), OAB inclusion criteria (minor differences exists between duration of $\mathrm{OAB}$ symptoms, frequency, and urgency), proportion of females in trials (all were $>50 \%$ ), and location of the trial (all studies except for one was in a noninstitutionalized setting). Two-thirds of RCTs and parent studies were of high-quality using the Jadad criteria.

Current guidelines recommend the use of any antimuscarinic or beta- 3 agonist as first-line drug treatment for $\mathrm{OAB}$ as efficacy among the two different classes are considered to be similar (Gormley et al., 2015; Thuroff et al., 2011). The approach to treating OAB in healthy older adults is similar to younger adults; however, frail older adults may have increased challenges as comorbidities, functional or cognitive deficits, and concomitant medications play a greater role in their management (Thuroff et al., 2011). Additionally, antimuscarinics in frail older adults may have a higher rate of AEs especially in the setting of polypharmacy (Geoffrion, 2012; Gormley et al., 2015).

In these analyses, AEs occurred at a higher rate in the antimuscarinic arms (53.8\%) compared to placebo $(41.7 \%)$ in this older adult population. Additionally, the discontinuation rate was also higher in the antimuscarinic group $(7.1 \%)$ versus placebo (5.0\%). This difference may be attributed to the higher rates of AEs. However, these studies are limited to up to 12 weeks of duration in patients who are willing to participate in a clinical trials and may not represent true rates of AEs and treatment discontinuation.

These findings show higher rates of anticholinergic AEs with antimuscarinics when compared to placebo in older adults. The overall rates of constipation and dry mouth in the antimuscarinic arms of these studies were $10 \%$ and $25 \%$, respectively. Subjects in the darifenacin (18.0\%) and solifenacin (15.4\%) arms had the highest rates of constipation. The rates of dry mouth were also highest in the fesoterodine (29.4\%), solifenacin (26.0\%), and darifenacin $(23.8 \%)$ arms. Compared to tolterodine ER (14.7\%), there was a two-fold increase in dry mouth in subjects receiving fesoterodine (31.3\%) suggesting a potential difference in rates of adverse outcomes between two antimuscarinics in older adults. This pooled analysis, which compared fesoterodine to tolterodine ER, suggested better efficacy in the fesoterodine arm with a statistically significant increase in dry mouth when compared to tolterodine ER (DuBeau et al., 2012). Fesoterodine is a pro-drug of tolterodine with doses of $4 \mathrm{mg}$ and $8 \mathrm{mg}$ compared to tolterodine with doses of $2 \mathrm{mg}$ and $4 \mathrm{mg}$. There was a 3 -fold and 4.8-fold increase rate of treatment discontinuation due to dry mouth observed in all antimuscarinics and fesoterodine, respectively, relative to placebo meaning dry mouth is bothersome enough to withdrawal from trials.

Older adults receiving fesoterodine $(2.5 \%)$ were more likely to experience dizziness compared to placebo (1\%). One alternative reason, beyond a true difference, for a higher rate of dizziness may be due to the increase sample size of subjects randomized to fesoterodine as $40 \%$ of subjects in the treatment arm were on fesoterodine in this analysis of older adults; therefore, it may be the only antimuscarinic with an adequate sample size needed to detect 
this difference. Conversely, there was no overall difference in the rate of dizziness in antimuscarinics compared to placebo. Another reason is the impact of age and/or frailty on the outcome of dizziness. DuBeau and colleagues assessed fesoterodine $(n=281)$ versus placebo $(n=281)$ in a vulnerable older adult population, defined by a Vulnerable Elders Survey score of 3 or more; however, the rate of dizziness in this population was $1.1 \%$ in the treatment arm (Dubeau et al., 2014). Kraus and colleagues showed an age-related increase in dizziness in older adults compared to younger adults which may suggest and age-related increase in dizziness (Kraus et al., 2010b). Additionally, there may be differences in identifying and classifying dizziness as safety outcomes were a part of the secondary analysis. Dizziness was the only significant cognitive- or functional-related AE in the study population. A previous meta-systematic review investigated the rate of central nervous system AEs, including dizziness, in older adults (Paquette et al., 2011). Similar, low rates of dizziness were identified in the antimuscarinics evaluated except of oxybutynin (3.0\%) and propiverine (3.2\%) (fesoterodine was not yet approved); however, within this analysis central nervous system AEs were not measured and may be underreported. Although the rate is low, dizziness may contribute to falls and fall-related fractures which may negatively impact quality of life.

Older adults randomized to fesoterodine were at 4-fold rate of dyspepsia compared to placebo (2.7\% versus $0.6 \%$ ) which was not noted in any other antimuscarinic sub-analysis. In a previous systematic review, the proportion of subjects receiving antimuscarinics (4.7\%) was higher than patients receiving placebo (2.1\%) (Chapple et al., 2008). This may be due to the anticholinergic properties of antimuscarinics causing reduced lower esophageal pressures which can present as dyspepsia (Peters, 1989). The difference identified in fesoterodine and not the other antimuscarinics may be due to the sample size of the fesoterodine population and the use of fesoterodine in a frail population.

Headache was not significant in the overall analysis; however, there was a two-fold rate of headache with tolterodine ER (2.7\%) compared to placebo (1.7\%) in three studies which contained information on approximately 1000 subjects in both treatment and placebo arms. Authors of a previous systematic review were unable to determine the mechanistic difference between tolterodine and other antimuscarinics in regards to headache and differences may be due to chance (Chapple et al., 2008).

Urinary retention was also significantly higher in the overall antimuscarinic group (1.3\%) compared to placebo $(0.3 \%)$, especially in subjects receiving fesoterodine $(1.7 \%)$ compared to placebo $(0.2 \%)$. In general, males are likely to be at a higher risk of urinary retention, especially at advancing age, due to the risk of benign prostatic enlargement (Emberton and Anson, 1999). However, one oxybutynin ER study of women only in the long-term care setting, two fesoterodine studies, and one trospium study reported urinary retention separately by sex (Dubeau et al., 2014; Lackner et al., 2008; Wagg et al., 2013; Zinner et al., 2002). The overall proportion of males $(0.7 \%)$ and females (1.1\%) with urinary retention was similar suggesting urinary retention is not an $\mathrm{AE}$ that affects only men; however, the risk of urinary retention in men is likely underestimated due to the exclusion of men with bladder outlet obstruction in these RCTs. 
There was nearly a two-fold increase in urinary tract infections in solifenacin (5.9\%) compared to placebo (3.1\%); however, there was no difference when evaluating all antimuscarinics versus placebo regarding urinary tract infections. The significant difference in solifenacin and placebo may due to an adequate sample size to detect a difference, random chance, or mechanistically, solifenacin is different from other antimuscarinics which may contribute to urinary tract infections.

\subsection{Limitations}

There are limitations to the study design. RCTs along with pooled and sub-analyses were the only study types included. These outcomes may not be generalizable to a clinical setting and may underrepresent the true rate of AEs and treatment discontinuations as there is the potential that subjects with higher risks of AEs and treatment discontinuations would be excluded for the study (Loke et al., 2007). Observational studies were not incorporated due to potential for inconsistent collection of AEs and treatment discontinuations across studies. Open-label studies with no comparator were not included for analysis as it may be difficult to discern between AEs and treatment discontinuations and the impact of aging. The duration of studies were 12 weeks or less; therefore, the authors cannot assess AEs and treatment discontinuations that may occur after continued use of antimuscarinics beyond 12 weeks. This may not allow enough time for a subject to develop and be identified with cognitive-related AEs as these may not present until beyond three months (Han et al., 2008). Many studies did not describe a standardized way of identifying or reporting AEs or treatment discontinuations. While the majority reported any AEs that occurred in at least 5\% of the subjects, there is a potential of not being able to identify all AEs across these studies, which may lead to an under identification of AEs and treatment discontinuations. Using the McHarm tool, the authors were able to be explicit on the differences in reporting AEs across studies. Similar terms, as previously described by Kessler, were used to combine potentially related AEs based on symptomatology (Kessler et al., 2011); however, no new significant AEs were detected. Efficacy was not analyzed in the systematic review and meta-analysis; therefore the authors cannot assess if the benefits of medications outweigh the risks of the various types of AEs. However, previous clinical guidelines suggest similar efficacy outcomes across antimuscarinics (Geoffrion, 2012; Gormley et al., 2015; Thuroff et al., 2011). Heterogeneity was not further explored beyond stratification of antimuscarinics as this was an exploratory analysis to identify AEs and treatment discontinuations that can be tested in future observational studies. Mirabegron and non-oral antimuscarinics (i.e., oxybutynin patch, oxybutynin gel) were not incorporated into these analyses. Only one study has assessed mirabegron in older adults (DuBeau et al., 2012) and no studies have evaluated non-oral antimuscarinics in older adults. These medications may have a differing AE profile. The authors did not assess for publication bias using funnel plots; however, since AEs were secondary outcomes, with the exception of one study (Herschorn et al., 2011), the publication of these studies were less dependent on positive or negative results in the safety outcomes compared to primary efficacy outcomes.

\subsection{Strengths}

Despite these limitations, there were many strengths to the study. This is the second review to aggregate RCTs, pooled analyses of RCTs, and sub-analyses of RCTs AEs of 
antimuscarinics (Paquette et al., 2011) and the first to perform an analysis of AEs beyond central-nervous system AEs with a large enough sample size to potentially detect differences in treatment groups that may not be able to be detected using individual studies. As RCTs were used in this analyses and not observational studies, the results are not impacted by channeling bias due to the random allocation of treatment and not on the preferential use of one medication over another based on clinical presentation and pre-existing comorbid conditions (Petri and Urquhart, 1991). The study also identified non-anticholinergic AEs which may not be routinely associated with antimuscarinics. Similar AEs were combined based on symptomatology to further explore AEs. Future studies to further explore less welldocumented antimuscarinic AEs using pharmacoepidemiological techniques should be performed. Properly attributing AEs to antimuscarinics may help identify alternative therapies and avoid prescribing cascades (Rochon and Gurwitz, 1997).

\section{Conclusion}

Treatment for overactive bladder using antimuscarinics in adults aged 65 or older resulted in significant increase risk of several AEs compared to placebo including both anticholinergic (e.g., dry mouth, constipation) and non-anticholinergic (e.g., dyspepsia, dizziness, headaches) AEs. Pharmacoepidemiology studies are needed to confirm AEs described in these analyses in a clinical setting. Differences in AEs, dry mouth, and discontinuation rates due to AEs were identified in head-to-head trials.

\section{Acknowledgments}

The authors would like to thank Graham A. Colditz, MD, DrPH, Carolyn Stoll, MPH, MSW, and Seth A. Strope, MD, MPH for their comments on the manuscript.

Research reported in this publication was supported by the Washington University Institute of Clinical and Translational Sciences grant UL1TR000448, sub-award KL2TR000450, from the National Center for Advancing Translational Sciences (NCATS) of the National Institutes of Health (NIH). The content is solely the responsibility of the authors and does not necessarily represent the official view of the NIH.

\section{Abbreviations}
AE adverse event
OAB overactive bladder
ER extended-release
IR immediate release
NNH number needed to harm
PRIMSA Preferred Reporting Items for Systematic Reviews and Meta-Analysis
RCT randomized controlled trial
SRMA systematic review and meta-analysis 


\section{References}

National Cancer Institute Common Terminology Criteria for Adverse Events v3.0 (CTCAE). 2009.

Appell RA, Sand P, Dmochowski R, Anderson R, Zinner N, Lama D, Roach M, Miklos J, Saltzstein D, Boone T, Staskin DR, Albrecht D. Prospective randomized controlled trial of extended-release oxybutynin chloride and tolterodine tartrate in the treatment of overactive bladder: results of the OBJECT Study. Mayo Clinic proceedings. 2001; 76:358-363. [PubMed: 11322350]

Benner JS, Nichol MB, Rovner ES, Jumadilova Z, Alvir J, Hussein M, Fanning K, Trocio JN, Brubaker L. Patient-reported reasons for discontinuing overactive bladder medication. BJU international. 2010; 105:1276-1282. [PubMed: 19912188]

Cardozo L, Lisec M, Millard R, van Vierssen Trip O, Kuzmin I, Drogendijk TE, Huang M, Ridder AM. Randomized, double-blind placebo controlled trial of the once daily antimuscarinic agent solifenacin succinate in patients with overactive bladder. The Journal of urology. 2004; 172:19191924. [PubMed: 15540755]

Chapple C, DuBeau C, Ebinger U, Rekeda L, Viegas A. Darifenacin treatment of patients >or $=65$ years with overactive bladder: results of a randomized, controlled, 12-week trial. Current medical research and opinion. 2007a; 23:2347-2358. [PubMed: 17706004]

Chapple C, Oelke M, Kaplan SA, Scholfield D, Arumi D, Wagg AS. Fesoterodine clinical efficacy and safety for the treatment of overactive bladder in relation to patient profiles: a systematic review. Current medical research and opinion. 2015; 31:1201-1243. [PubMed: 25798911]

Chapple C, Van Kerrebroeck P, Tubaro A, Haag-Molkenteller C, Forst HT, Massow U, Wang J, Brodsky M. Clinical efficacy, safety, and tolerability of once-daily fesoterodine in subjects with overactive bladder. European urology. 2007b; 52:1204-1212. [PubMed: 17651893]

Chapple CR, Arano P, Bosch JL, De Ridder D, Kramer AE, Ridder AM. Solifenacin appears effective and well tolerated in patients with symptomatic idiopathic detrusor overactivity in a placebo- and tolterodine-controlled phase 2 dose-finding study. BJU international. 2004a; 93:71-77. [PubMed: 14678372]

Chapple CR, Khullar V, Gabriel Z, Muston D, Bitoun CE, Weinstein D. The effects of antimuscarinic treatments in overactive bladder: an update of a systematic review and meta-analysis. European urology. 2008; 54:543-562. [PubMed: 18599186]

Chapple CR, Martinez-Garcia R, Selvaggi L, Toozs-Hobson P, Warnack W, Drogendijk T, Wright DM, Bolodeoku J. A comparison of the efficacy and tolerability of solifenacin succinate and extended release tolterodine at treating overactive bladder syndrome: results of the STAR trial. European urology. 2005; 48:464-470. [PubMed: 15990220]

Chapple CR, Rechberger T, Al-Shukri S, Meffan P, Everaert K, Huang M, Ridder A. Randomized, double-blind placebo- and tolterodine-controlled trial of the once-daily antimuscarinic agent solifenacin in patients with symptomatic overactive bladder. BJU international. 2004b; 93:303310. [PubMed: 14764127]

Cui Y, Zong H, Yang C, Yan H, Zhang Y. The efficacy and safety of mirabegron in treating OAB: a systematic review and meta-analysis of phase III trials. International urology and nephrology. 2014; 46:275-284. [PubMed: 23896942]

Dmochowski RR, Sand PK, Zinner NR, Staskin DR. Trospium $60 \mathrm{mg}$ once daily (QD) for overactive bladder syndrome: results from a placebo-controlled interventional study. Urology. 2008; 71:449_ 454. [PubMed: 18342185]

Dubeau CE, Kraus SR, Griebling TL, Newman DK, Wyman JF, Johnson TM 2nd, Ouslander JG, Sun F, Gong J, Bavendam T. Effect of fesoterodine in vulnerable elderly subjects with urgency incontinence: a double-blind, placebo controlled trial. The Journal of urology. 2014; 191:395-404. [PubMed: 23973522]

DuBeau CE, Morrow JD, Kraus SR, Creanga D, Bavendam T. Efficacy and tolerability of fesoterodine versus tolterodine in older and younger subjects with overactive bladder: a post hoc, pooled analysis from two placebo-controlled trials. Neurourology and urodynamics. 2012; 31:1258-1265. [PubMed: 22907761]

Emberton M, Anson K. Acute urinary retention in men: an age old problem. BMJ (Clinical research ed). 1999; 318:921-925. 
Foote J, Glavind K, Kralidis G, Wyndaele JJ. Treatment of overactive bladder in the older patient: pooled analysis of three phase III studies of darifenacin, an M3 selective receptor antagonist. European urology. 2005; 48:471-477. [PubMed: 15990219]

Geoffrion R. Treatments for overactive bladder: focus on pharmacotherapy. Journal of obstetrics and gynaecology Canada : JOGC = Journal d'obstetrique et gynecologie du Canada : JOGC. 2012; 34:1092-1101.

Gomelsky A. Urinary incontinence in the elderly female. Annals of Long-Term Care. 2009; 17:41-45.

Gormley EA, Lightner DJ, Faraday M, Vasavada SP. Diagnosis and treatment of overactive bladder (non-neurogenic) in adults: AUA/SUFU guideline amendment. The Journal of urology. 2015; 193:1572-1580. [PubMed: 25623739]

Griebling TL, Kraus SR, Richter HE, Glasser DB, Carlsson M. Tolterodine extended release is well tolerated in older subjects. International journal of clinical practice. 2009; 63:1198-1204. [PubMed: 19624787]

Han L, Agostini JV, Allore HG. Cumulative anticholinergic exposure is associated with poor memory and executive function in older men. Journal of the American Geriatrics Society. 2008; 56:22032210. [PubMed: 19093918]

Herbison P, Hay-Smith J, Ellis G, Moore K. Effectiveness of anticholinergic drugs compared with placebo in the treatment of overactive bladder: systematic review. BMJ (Clinical research ed). $2003 ; 326: 841-844$.

Herschorn S, Barkin J, Castro-Diaz D, Frankel JM, Espuna-Pons M, Gousse AE, Stolzel M, Martin N, Gunther A, Van Kerrebroeck P. A phase III, randomized, double-blind, parallel-group, placebocontrolled, multicentre study to assess the efficacy and safety of the beta(3) adrenoceptor agonist, mirabegron, in patients with symptoms of overactive bladder. Urology. 2013; 82:313-320. [PubMed: 23769122]

Herschorn S, Pommerville P, Stothers L, Egerdie B, Gajewski J, Carlson K, Radomski S, Drutz H, Schulz J, Barkin J, Hirshberg E, Corcos J. Tolerability of solifenacin and oxybutynin immediate release in older ( $>65$ years) and younger $(</=65$ years) patients with overactive bladder: subanalysis from a Canadian, randomized, double-blind study. Current medical research and opinion. 2011; 27:375-382. [PubMed: 21175373]

Herschorn S, Stothers L, Carlson K, Egerdie B, Gajewski JB, Pommerville P, Schulz J, Radomski S, Drutz H, Barkin J, Paradiso-Hardy F. Tolerability of $5 \mathrm{mg}$ solifenacin once daily versus $5 \mathrm{mg}$ oxybutynin immediate release 3 times daily: results of the VECTOR trial. The Journal of urology. 2010a; 183:1892-1898. [PubMed: 20303119]

Herschorn S, Swift S, Guan Z, Carlsson M, Morrow JD, Brodsky M, Gong J. Comparison of fesoterodine and tolterodine extended release for the treatment of overactive bladder: a head-tohead placebo-controlled trial. BJU international. 2010b; 105:58-66. [PubMed: 20132103]

Huang W, Zong H, Zhou X, Zhang Y. Efficacy and safety of imidafenacin for overactive bladder in adult: a systematic review and meta-analysis. International urology and nephrology. 2015; 47:457464. [PubMed: 25636812]

Jadad AR, Moore RA, Carroll D, Jenkinson C, Reynolds DJ, Gavaghan DJ, McQuay HJ. Assessing the quality of reports of randomized clinical trials: is blinding necessary? Controlled clinical trials. 1996; 17:1-12. [PubMed: 8721797]

Kaplan SA, Schneider T, Foote JE, Guan Z, Carlsson M, Gong J. Superior efficacy of fesoterodine over tolterodine extended release with rapid onset: a prospective, head-to-head, placebo-controlled trial. BJU international. 2011; 107:1432-1440. [PubMed: 20860717]

Kessler TM, Bachmann LM, Minder C, Lohrer D, Umbehr M, Schunemann HJ, Kessels AG. Adverse event assessment of antimuscarinics for treating overactive bladder: a network meta-analytic approach. PloS one. 2011; 6:e16718. [PubMed: 21373193]

Khullar V, Amarenco G, Angulo JC, Cambronero J, Hoye K, Milsom I, Radziszewski P, Rechberger T, Boerrigter P, Drogendijk T, Wooning M, Chapple C. Efficacy and tolerability of mirabegron, a beta(3)-adrenoceptor agonist, in patients with overactive bladder: results from a randomised European-Australian phase 3 trial. European urology. 2013; 63:283-295. [PubMed: 23182126]

Kraus SR, Bavendam T, Brake T, Griebling TL. Vulnerable elderly patients and overactive bladder syndrome. Drugs \& aging. 2010a; 27:697-713. [PubMed: 20809661] 
Kraus SR, Ruiz-Cerda JL, Martire D, Wang JT, Wagg AS. Efficacy and tolerability of fesoterodine in older and younger subjects with overactive bladder. Urology. 2010b; 76:1350-1357. [PubMed: 20974482]

Lackner TE, Wyman JF, McCarthy TC, Monigold M, Davey C. Randomized, placebo-controlled trial of the cognitive effect, safety, and tolerability of oral extended-release oxybutynin in cognitively impaired nursing home residents with urge urinary incontinence. Journal of the American Geriatrics Society. 2008; 56:862-870. [PubMed: 18410326]

Loke YK, Price D, Herxheimer A. Systematic reviews of adverse effects: framework for a structured approach. BMC medical research methodology. 2007; 7:32. [PubMed: 17615054]

Luo D, Liu L, Han P, Wei Q, Shen H. Solifenacin for overactive bladder: a systematic review and meta-analysis. International urogynecology journal. 2012; 23:983-991. [PubMed: 22310924]

Macdiarmid SA. Maximizing the treatment of overactive bladder in the elderly. Reviews in urology. 2008; 10:6-13. [PubMed: 18470275]

Madhuvrata P, Cody JD, Ellis G, Herbison GP, Hay-Smith EJ. Which anticholinergic drug for overactive bladder symptoms in adults. The Cochrane database of systematic reviews. 2012; 1:Cd005429. [PubMed: 22258963]

Malone-Lee JG, Walsh JB, Maugourd MF. Tolterodine: a safe and effective treatment for older patients with overactive bladder. Journal of the American Geriatrics Society. 2001; 49:700-705. [PubMed: 11454106]

Milsom I, Stewart W, Thuroff J. The prevalence of overactive bladder. The American journal of managed care. 2000; 6:S565-573. [PubMed: 11183899]

Minassian VA, Ross S, Sumabat O, Lovatsis D, Pascali D, Al-Badr A, Alarab M, Drutz HP. Randomized trial of oxybutynin extended versus immediate release for women aged 65 and older with overactive bladder: lessons learned from conducting a trial. Journal of obstetrics and gynaecology Canada : JOGC = Journal d'obstetrique et gynecologie du Canada : JOGC. 2007; 29:726-732.

Nitti VW, Auerbach S, Martin N, Calhoun A, Lee M, Herschorn S. Results of a randomized phase III trial of mirabegron in patients with overactive bladder. The Journal of urology. 2013; 189:13881395. [PubMed: 23079373]

Nitti VW, Dmochowski R, Sand PK, Forst HT, Haag-Molkenteller C, Massow U, Wang J, Brodsky M, Bavendam T. Efficacy, safety and tolerability of fesoterodine for overactive bladder syndrome. The Journal of urology. 2007; 178:2488-2494. [PubMed: 17937959]

Novara G, Galfano A, Secco S, D'Elia C, Cavalleri S, Ficarra V, Artibani W. A systematic review and meta-analysis of randomized controlled trials with antimuscarinic drugs for overactive bladder. European urology. 2008; 54:740-763. [PubMed: 18632201]

Paquette A, Gou P, Tannenbaum C. Systematic review and meta-analysis: do clinical trials testing antimuscarinic agents for overactive bladder adequately measure central nervous system adverse events? Journal of the American Geriatrics Society. 2011; 59:1332-1339. [PubMed: 21718264]

Peters NL. Snipping the thread of life. Antimuscarinic side effects of medications in the elderly. Archives of internal medicine. 1989; 149:2414-2420. [PubMed: 2684071]

Petri H, Urquhart J. Channeling bias in the interpretation of drug effects. Statistics in medicine. 1991; 10:577-581. [PubMed: 2057656]

Reynolds WS, McPheeters M, Blume J, Surawicz T, Worley K, Wang L, Hartmann K. Comparative Effectiveness of Anticholinergic Therapy for Overactive Bladder in Women: A Systematic Review and Meta-analysis. Obstetrics and gynecology. 2015; 125:1423-1432. [PubMed: 26000514]

Rochon PA, Gurwitz JH. Optimising drug treatment for elderly people: the prescribing cascade. BMJ (Clinical research ed). 1997; 315:1096-1099.

Roxburgh C, Cook J, Dublin N. Anticholinergic drugs versus other medications for overactive bladder syndrome in adults. The Cochrane database of systematic reviews. 2007:Cd003190.

Sand PK, Johnson TM II, Rovner ES, Ellsworth PI, Oefelein MG, Staskin DR. Trospium chloride once-daily extended release is efficacious and tolerated in elderly subjects (aged $>/=75$ years) with overactive bladder syndrome. BJU international. 2011; 107:612-620. [PubMed: 20707790] 
Sand PK, Miklos J, Ritter H, Appell R. A comparison of extended-release oxybutynin and tolterodine for treatment of overactive bladder in women. International urogynecology journal and pelvic floor dysfunction. 2004; 15:243-248. [PubMed: 15517668]

Santaguida PL, Raina P. The Development of the McHarm Quality Assessment Scale for adverse events: Delphi Consensus on important criteria for evaluating harms. 2008

Scheife R, Takeda M. Central nervous system safety of anticholinergic drugs for the treatment of overactive bladder in the elderly. Clinical therapeutics. 2005; 27:144-153. [PubMed: 15811477]

Staskin D, Sand P, Zinner N, Dmochowski R. Once daily trospium chloride is effective and well tolerated for the treatment of overactive bladder: results from a multicenter phase III trial. The Journal of urology. 2007; 178:978-983. discussion 983-974. [PubMed: 17632131]

Sternberg SA, Wershof Schwartz A, Karunananthan S, Bergman H, Mark Clarfield A. The identification of frailty: a systematic literature review. Journal of the American Geriatrics Society. 2011; 59:2129-2138. [PubMed: 22091630]

Szonyi G, Collas DM, Ding YY, Malone-Lee JG. Oxybutynin with bladder retraining for detrusor instability in elderly people: a randomized controlled trial. Age and ageing. 1995; 24:287-291. [PubMed: 7484484]

Thuroff JW, Abrams P, Andersson KE, Artibani W, Chapple CR, Drake MJ, Hampel C, Neisius A, Schroder A, Tubaro A. EAU guidelines on urinary incontinence. European urology. 2011; 59:387400. [PubMed: 21130559]

Tubaro A. Defining overactive bladder: epidemiology and burden of disease. Urology. 2004; 64:2-6.

Van Kerrebroeck P, Kreder K, Jonas U, Zinner N, Wein A. Tolterodine once-daily: superior efficacy and tolerability in the treatment of the overactive bladder. Urology. 2001; 57:414-421. [PubMed: 11248608]

Wagg A, Cardozo L, Nitti VW, Castro-Diaz D, Auerbach S, Blauwet MB, Siddiqui E. The efficacy and tolerability of the beta3-adrenoceptor agonist mirabegron for the treatment of symptoms of overactive bladder in older patients. Age and ageing. 2014; 43:666-675. [PubMed: 24610862]

Wagg A, Khullar V, Marschall-Kehrel D, Michel MC, Oelke M, Darekar A, Bitoun CE, Weinstein D, Osterloh I. Flexible-dose fesoterodine in elderly adults with overactive bladder: results of the randomized, double-blind, placebo-controlled study of fesoterodine in an aging population trial. Journal of the American Geriatrics Society. 2013; 61:185-193. [PubMed: 23350833]

Wagg A, Verdejo C, Molander U. Review of cognitive impairment with antimuscarinic agents in elderly patients with overactive bladder. International journal of clinical practice. $2010 ; 64: 1279$ 1286. [PubMed: 20529135]

Wagg A, Wyndaele JJ, Sieber P. Efficacy and tolerability of solifenacin in elderly subjects with overactive bladder syndrome: a pooled analysis. The American journal of geriatric pharmacotherapy. 2006; 4:14-24. [PubMed: 16730617]

Wu T, Duan X, Cao CX, Peng CD, Bu SY, Wang KJ. The role of mirabegron in overactive bladder: a systematic review and meta-analysis. Urologia internationalis. 2014; 93:326-337. [PubMed: 25115445]

Wyndaele JJ, Schneider T, MacDiarmid S, Scholfield D, Arumi D. Flexible dosing with fesoterodine 4 and $8 \mathrm{mg}$ : a systematic review of data from clinical trials. International journal of clinical practice. 2014; 68:830-840. [PubMed: 24754814]

Zinner NR, Mattiasson A, Stanton SL. Efficacy, safety, and tolerability of extended-release once-daily tolterodine treatment for overactive bladder in older versus younger patients. Journal of the American Geriatrics Society. 2002; 50:799-807. [PubMed: 12028164]

\section{Appendix 1: Complete Search Strategy}

\section{PubMed}

(“aged" OR “Aged”[Mesh] OR "elderly" OR "aged, 80 and over”[Mesh] OR "aged, 80 and over" OR "oldest old" OR "nonagenarian*” OR "octogenarian*” OR "centenarian*” OR "frail elderly"[Mesh] OR "frail elder*" OR "functionally-impaired elderly" OR "frail older 
adult*" OR "retire*” OR "septuagenarian*” OR "sexagenarian*” OR "geriatric*” OR "senior citizen") AND ("Muscarinic Antagonists”[Mesh] OR “Antagonists, Muscarinic" OR "Antimuscarinics" or "Antimuscarinic Agents" OR "Agents, Antimuscarinic" OR "Cholinergic Muscarinic Antagonists" OR "Antagonists, Cholinergic Muscarinic" OR "Muscarinic Antagonists, Cholinergic" OR "Antimuscarinic agent" OR "antimuscarinic drug" OR "muscarinic blocker" OR "muscarinic blocking agent" OR "muscarinic receptor antagonist" OR "muscarinic receptor blocker" OR "oxybutynin" [Supplementary Concept] OR “oxybutynin” OR "K9P6MC7092" OR "Zatur" OR "Contimin” OR "Cystonorm” OR "Ditropan” OR "Dridase" OR "Cystrin" OR "Dresplan" OR "Driptane" OR "gelnique" OR "Gen-Oxybutynin” OR "Novo-Oxybutynin" OR "Nu-Oxybutyn” OR "Oxyb AbZ” OR "Oxybutin Holsten" OR "Oxybuton" OR "Oxybutynin AL" OR "Oxybutynin AZU” OR "Oxybutynin Heumann" OR "Oxybutynin Hexal” OR "oxybutynin hydrochloride” OR "oxybutynin chloride" OR "Oxybutynin Stada" OR "oxybutynin von ct" OR "OxybutyninPuren" OR "Oxybutynin-ratiopharm" OR "Oxymedin" OR "Oxytrol” OR "PMSOxybutynin" OR "Pollakisu" OR "Renamel” OR "Ryol" OR "Spasmex" OR "Oxybutynin" OR "Spasyt" OR "Tavor" OR "Oxybugamma" OR "Apo-Oxybutynin" OR "tolterodine" [Supplementary Concept] OR "WHE7A56U7K" OR "PHA-686464B" OR "Urotrol” OR "Detrol" OR "Unidet" OR "Detrusitol" OR "tolterodine tartrate" OR "trospium chloride" [Supplementary Concept] OR "trospium chloride" OR "1E6682427E" OR "azoniaspiro compound XVII" OR OR "Spasmex" OR "Uraton" OR "Spasmolyt" OR "Spasmo-Urgenin TC" OR "Spasmo-lyt" OR "Spasmo-Rhoival TC" OR "Trospi” OR "Ceris" OR "Uraplex" OR "Spasmo-Urgenin" OR "Sanctura" OR "solifenacin" [Supplementary Concept] OR "solifenacin" OR “A8910SQJ1U” OR "YM905" OR "YM 905" OR "solifenacin succinate" OR "vesicare" OR "darifenacin" [Supplementary Concept] OR "darifenacin" OR "darifenacin" OR "darifenacine" OR "darifenacin hydrochloride" OR "Enablex" OR "UK-88525" OR "darifenacin hydrobromide" OR "Emselex" OR “APG9819VLM" OR "fesoterodine" [Supplementary Concept] OR "fesoterodine" OR "621G617227” OR "toviaz" OR "fesoterodine fumarate" OR Mirabegron" OR "mirabegron" [Supplementary Concept] OR "MVR3JL3B2V" OR "Betanis" OR "YM 178” OR "YM-178” OR "Adrenergic beta-3 Receptor Agonists")

\section{EMBASE}

('aged'/exp OR 'aged' OR 'frail elderly'/exp OR 'very elderly'/exp OR ‘very elderly' OR 'geriatrics'/exp OR 'geriatrics' OR 'geriatric patient'/exp OR 'geriatric patient' OR 'elderly' OR 'aged, 80 and over' OR 'oldest old' OR 'nonagenarian' OR 'nonagenarians' OR 'octogenarian' OR 'octogenarians' OR 'centenarian' OR 'centenarians' OR 'frail elder' OR 'frail elders' OR 'frail elderly' OR 'functionally-impaired elderly' OR 'frail older adult' OR 'frail older adults' OR 'retiree' OR 'septuagenarian' OR ‘septuagenarians' OR 'sexagenarian' OR 'sexagenarians' OR 'geriatric' OR 'senior citizen') AND ('muscarinic receptor blocking agent'/exp OR 'Antagonists, Muscarinic' OR 'Antimuscarinics' or 'Antimuscarinic Agents' OR 'Agents, Antimuscarinic' OR 'Cholinergic Muscarinic Antagonists' OR 'Antagonists, Cholinergic Muscarinic' OR 'Muscarinic Antagonists, Cholinergic' OR 'Antimuscarinic agent' OR 'antimuscarinic drug' OR 'muscarinic antagonist' OR 'muscarinic antagonists' OR 'muscarinic blocker' OR 'muscarinic blocking 
agent' OR 'muscarinic receptor antagonist' OR 'muscarinic receptor blocker' OR 'muscarinolytic agent' OR 'oxybutynin'/exp OR 'anturol' OR 'cystonorm' OR 'cystrin' OR 'delifon' OR ‘ditropan' OR 'ditropan xl' OR ‘diutropin' OR 'dridase' OR 'driptane' OR 'esoxybutynin' OR 'esoxybutynin chloride' OR 'frenurin' OR 'gelnique' OR 'iliaden' OR 'kentera' OR ‘kl 007' OR 'kl007' OR 'lenditro' OR 'lyrinel xl' OR ‘mj 4309 1' OR 'mj 43091' OR 'mj43091' OR 'mutum cr' OR 'nefryl' OR ‘novitropan' OR ‘oxibutinin' OR 'oxibutynin' OR ‘oxyban' OR ‘oxybutinin' OR ‘oxybutinin chloride' OR ‘oxybutyin' OR 'oxybutyin chloride' OR 'oxybutynin chlorhydrate' OR 'oxybutynin chloride' OR 'oxybutynin hydrochloride' OR 'oxytrol' OR 'oxytrol for women' OR 'oyrobin' OR 'pollakis' OR 'reteven' OR 'tropan' OR 'uricont' OR 'uroflax' OR 'urotrol' OR 'zatur ge' OR 'Zatur' OR 'Contimin' OR ‘Cystonorm' OR 'Dresplan' OR 'Driptane' OR 'gelnique' OR 'Gen-Oxybutynin' OR 'Novo-Oxybutynin' OR ‘Nu-Oxybutyn' OR 'Oxyb AbZ' OR 'Oxybutin Holsten' OR 'Oxybuton' OR 'Oxybutynin AL' OR 'Oxybutynin AZU' OR 'Oxybutynin Heumann' OR 'Oxybutynin Hexal' OR 'oxybutynin hydrochloride' OR 'Oxybutynin Stada' OR 'oxybutynin von ct' OR 'Oxybutynin-Puren' OR 'Oxybutyninratiopharm' OR 'Oxymedin' OR 'Pollakisu' OR 'Renamel' OR 'Ryol' OR 'Spasmex' OR 'Spasyt' OR 'Tavor' OR 'Oxybugamma' OR 'Apo-Oxybutynin' OR ‘tolterodine'/exp OR ‘detrol' OR ‘detrol la' OR ‘detrusitol' OR 'pnu 200583' OR 'pnu200583' OR 'tolterodine tartrate' OR 'WHE7A56U7K' OR 'PHA-686464B' OR 'Urotrol' OR 'Unidet' OR 'trospium chloride'/exp OR 'regurin' OR 'sanctura' OR 'sanctura xr' OR 'spasmex' OR 'spasmo urgenin' OR 'spasmo-lyt' OR 'spasmolyt' OR 'spasmourgenin' OR 'trospium chloride' OR '1E6682427E' OR 'azoniaspiro compound XVII' OR 'Spasmex' OR 'Uraton' OR 'Spasmo-Urgenin TC' OR 'Spasmo-Rhoival TC' OR 'Trospi' OR 'Ceris' OR 'Uraplex' OR 'Spasmo-Urgenin' OR 'Sanctura' OR 'solifenacin'/exp OR 'solifenacin succinate' OR 'vesicare' OR ‘vesikur' OR ‘ym 53705' OR ‘ym 905' OR ‘ym53705' OR ‘ym905' OR 'A8910SQJ1U' OR 'darifenacin'/exp OR 'darifenacin hydrobromide' OR 'emselex' OR 'enablex' OR ‘uk 88525' OR 'uk 88525 04' OR ‘uk 88525-04' OR 'uk88525' OR 'uk88525 04' OR 'uk88525-04' OR 'darifenacine' OR 'darifenacin hydrochloride' OR 'UK-88525' OR 'APG9819VLM' OR 'fesoterodine'/exp OR 'fesoterodine fumarate' OR 'fesoterodine hydrogen fumarate' OR 'spm 907' OR 'spm907' OR 'toviaz' OR '621G617227' OR 'mirabegron'/exp OR 'betanis' OR 'betmiga' OR 'myrbetriq' OR 'sc 211912' OR 'sc211912' OR ‘ym 178' OR ‘ym178' OR 'MVR3JL3B2V' OR 'YM-178' OR 'Adrenergic beta-3 Receptor Agonists')

\section{SCOPUS}

(TITLE-ABS-KEY(“aged”) OR TITLE-ABS-KEY(“elderly”) OR TITLE-ABS-KEY(“aged, 80 and over") OR TITLE-ABS-KEY(“oldest old") OR TITLE-ABS-KEY("nonagenarian*”) OR TITLE-ABS-KEY(“octogenarian*”) OR TITLE-ABS-KEY(“centenarian*”) OR TITLE-ABS-KEY("frail elder*”) OR TITLE-ABS-KEY("functionally-impaired elderly") OR TITLE-ABS-KEY("frail older adult*") OR TITLE-ABS-KEY("retire*”) OR TITLEABS-KEY("septuagenarian*”) OR TITLE-ABS-KEY("sexagenarian*”) OR TITLE-ABSKEY(“geriatric*”) OR TITLE-ABS-KEY("senior citizen") OR TITLE-ABS-KEY(“very elderly") OR TITLE-ABS-KEY(“geriatric patient”)) AND (TITLE-ABS-KEY("Muscarinic Antagonists”) OR TITLE-ABS-KEY(“Antagonists, Muscarinic”) OR TITLE-ABS- 
KEY(“Antimuscarinics”) OR TITLE-ABS-KEY (“Antimuscarinic Agents”) OR TITLEABS-KEY(“Agents, Antimuscarinic") OR TITLE-ABS-KEY(“Cholinergic Muscarinic Antagonists") OR TITLE-ABS-KEY(“Antagonists, Cholinergic Muscarinic”) OR TITLEABS-KEY("Muscarinic Antagonists, Cholinergic") OR TITLE-ABS-KEY(“Antimuscarinic agent") OR TITLE-ABS-KEY(“antimuscarinic drug") OR TITLE-ABS-KEY(“muscarinic blocker") OR TITLE-ABS-KEY(“muscarinic blocking agent”) OR TITLE-ABS-

KEY("muscarinic receptor antagonist") OR TITLE-ABS-KEY("muscarinic receptor blocker") OR TITLE-ABS-KEY(“oxybutynin”) OR TITLE-ABS-KEY(“K9P6MC7092”) OR TITLE-ABS-KEY(“Zatur”) OR TITLE-ABS-KEY(“Contimin”) OR TITLE-ABSKEY(“Cystonorm”) OR TITLE-ABS-KEY(“Ditropan”) OR TITLE-ABS-KEY(“Dridase”) OR TITLE-ABS-KEY(“Cystrin”) OR TITLE-ABS-KEY(“Dresplan”) OR TITLE-ABSKEY("Driptane") OR TITLE-ABS-KEY("gelnique”) OR TITLE-ABS-KEY(“GenOxybutynin”) OR TITLE-ABS-KEY(“Novo-Oxybutynin”) OR TITLE-ABS-KEY(“NuOxybutyn”) OR TITLE-ABS-KEY(“'Oxyb AbZ”) OR TITLE-ABS-KEY(“Oxybutin Holsten”) OR TITLE-ABS-KEY(“Oxybuton”) OR TITLE-ABS-KEY(“Oxybutynin AL”) OR TITLE-ABS-KEY(“Oxybutynin AZU”) OR TITLE-ABS-KEY(“Oxybutynin Heumann”) OR TITLE-ABS-KEY(“Oxybutynin Hexal”) OR TITLE-ABSKEY(“oxybutynin hydrochloride") OR TITLE-ABS-KEY(“oxybutynin chloride”) OR TITLE-ABS-KEY(“Oxybutynin Stada”) OR TITLE-ABS-KEY(“oxybutynin von ct”) OR TITLE-ABS-KEY(“Oxybutynin-Puren”) OR TITLE-ABS-KEY(“Oxybutynin-ratiopharm”) OR TITLE-ABS-KEY(“Oxymedin”) OR TITLE-ABS-KEY(“Oxytrol”) OR TITLE-ABSKEY(“PMS-Oxybutynin”) OR TITLE-ABS-KEY(“Pollakisu”) OR TITLE-ABSKEY("Renamel”) OR TITLE-ABS-KEY(“Ryol”) OR TITLE-ABS-KEY(“Spasmex”) OR TITLE-ABS-KEY(“Oxybutynin”) OR TITLE-ABS-KEY(“Spasyt”) OR TITLE-ABSKEY(“Tavor”) OR TITLE-ABS-KEY(“Oxybugamma”) OR TITLE-ABS-KEY(“ApoOxybutynin”) OR TITLE-ABS-KEY(“tolterodine”) OR TITLE-ABSKEY(“WHE7A56U7K”) OR TITLE-ABS-KEY(“PHA-686464B”) OR TITLE-ABSKEY(“Urotrol”) OR TITLE-ABS-KEY(“Detrol”) OR TITLE-ABS-KEY(“Unidet”) OR TITLE-ABS-KEY(“Detrusitol”) OR TITLE-ABS-KEY("tolterodine tartrate”) OR TITLEABS-KEY("trospium chloride") OR TITLE-ABS-KEY("trospium chloride") OR TITLEABS-KEY(“1E6682427E”) OR TITLE-ABS-KEY(“azoniaspiro compound XVII”) OR TITLE-ABS-KEY(“Spasmex”) OR TITLE-ABS-KEY(“Uraton”) OR TITLE-ABSKEY(“Spasmolyt”) OR TITLE-ABS-KEY(“Spasmo-Urgenin TC”) OR TITLE-ABSKEY(“Spasmo-lyt”) OR TITLE-ABS-KEY(“Spasmo-Rhoival TC”) OR TITLE-ABSKEY(“Trospi”) OR TITLE-ABS-KEY(“Ceris”) OR TITLE-ABS-KEY(“Uraplex”) OR TITLE-ABS-KEY(“Spasmo-Urgenin”) OR TITLE-ABS-KEY(“Sanctura”) OR TITLEABS-KEY(“solifenacin”) OR TITLE-ABS-KEY(“solifenacin”) OR TITLE-ABSKEY(“A8910SQJ1U”) OR TITLE-ABS-KEY(“YM905”) OR TITLE-ABS-KEY(“YM 905”) OR TITLE-ABS-KEY(“solifenacin succinate”) OR TITLE-ABS-KEY(“vesicare”) OR TITLE-ABS-KEY(“darifenacin”) OR TITLE-ABS-KEY(“darifenacin”) OR TITLEABS-KEY(“darifenacin”) OR TITLE-ABS-KEY(“darifenacine”) OR TITLE-ABSKEY(“darifenacin hydrochloride”) OR TITLE-ABS-KEY(“Enablex”) OR TITLE-ABSKEY(“UK-88525”) OR TITLE-ABS-KEY(“darifenacin hydrobromide”) OR TITLE-ABSKEY(“Emselex") OR TITLE-ABS-KEY(“APG9819VLM”) OR TITLE-ABSKEY("fesoterodine”) OR TITLE-ABS-KEY("fesoterodine”) OR TITLE-ABS- 
KEY(“621G617227”) OR TITLE-ABS-KEY(“toviaz”) OR TITLE-ABS-KEY(“fesoterodine fumarate”) OR TITLE-ABS-KEY(“Adrenergic beta-3 Receptor Agonists”) OR TITLEABS-KEY("Mirabegron") OR TITLE-ABS-KEY("Mirabegron") OR TITLE-ABSKEY(“MVR3JL3B2V”) OR TITLE-ABS-KEY(“Betanis") OR TITLE-ABS-KEY(“YM 178”) OR TITLE-ABS-KEY(“YM-178”))

\section{COCHRAN RCTS}

("aged" OR “Aged" OR “elderly" OR "aged, 80 and over" OR "aged, 80 and over" OR "oldest old" OR "nonagenarian*” OR "octogenarian*” OR "centenarian*” OR "frail elderly"[Mesh] OR "frail elder*" OR "functionally-impaired elderly" OR "frail older adult*” OR "retire*” OR "septuagenarian*” OR "sexagenarian*” OR "geriatric*” OR "senior citizen") AND ("Muscarinic Antagonists" OR "Antagonists, Muscarinic" OR "Antimuscarinics" or "Antimuscarinic Agents" OR "Agents, Antimuscarinic" OR "Cholinergic Muscarinic Antagonists" OR "Antagonists, Cholinergic Muscarinic" OR "Muscarinic Antagonists, Cholinergic" OR "Antimuscarinic agent” OR "antimuscarinic drug" OR "muscarinic blocker" OR "muscarinic blocking agent" OR "muscarinic receptor antagonist" OR "muscarinic receptor blocker" OR "oxybutynin" OR “oxybutynin” OR "K9P6MC7092" OR "Zatur" OR "Contimin" OR "Cystonorm" OR "Ditropan" OR "Dridase" OR "Cystrin" OR "Dresplan" OR "Driptane" OR "gelnique" OR "GenOxybutynin" OR "Novo-Oxybutynin" OR "Nu-Oxybutyn" OR "Oxyb AbZ” OR "Oxybutin Holsten" OR "Oxybuton" OR "Oxybutynin AL" OR "Oxybutynin AZU” OR "Oxybutynin Heumann" OR "Oxybutynin Hexal” OR "oxybutynin hydrochloride” OR "oxybutynin chloride" OR "Oxybutynin Stada" OR "oxybutynin von ct" OR "Oxybutynin-Puren” OR "Oxybutynin-ratiopharm" OR "Oxymedin" OR "Oxytrol” OR "PMS-Oxybutynin" OR "Pollakisu” OR "Renamel” OR "Ryol” OR "Spasmex" OR "Oxybutynin” OR "Spasyt” OR "Tavor" OR "Oxybugamma" OR "Apo-Oxybutynin" OR "tolterodine" OR "WHE7A56U7K" OR "PHA-686464B" OR "Urotrol” OR "Detrol” OR "Unidet" OR "Detrusitol" OR "tolterodine tartrate" OR "trospium chloride" OR "trospium chloride" OR “1E6682427E” OR "azoniaspiro compound XVII" OR OR "Spasmex” OR "Uraton” OR "Spasmolyt" OR "Spasmo-Urgenin TC" OR "Spasmo-lyt" OR "Spasmo-Rhoival TC" OR "Trospi” OR "Ceris" OR "Uraplex" OR "Spasmo-Urgenin" OR "Sanctura" OR "solifenacin" OR "solifenacin" OR “A8910SQJ1U” OR "YM905" OR "YM 905" OR "solifenacin succinate" OR "vesicare" OR "darifenacin" OR "darifenacin" OR "darifenacin" OR "darifenacine" OR "darifenacin hydrochloride" OR "Enablex" OR "UK-88525" OR "darifenacin hydrobromide" OR "Emselex" OR "APG9819VLM" OR "fesoterodine" OR "fesoterodine" OR "621G617227" OR "toviaz" OR "fesoterodine fumarate" OR "Mirabegron" OR "mirabegron" OR "MVR3JL3B2V” OR "Betanis" OR "YM 178” OR "YM-178" OR "Adrenergic beta-3 Receptor Agonists")

\section{Appendix 2. McMaster Tool for Assessing Quality of Harms Assessments and Reporting in Study Reports (McHarm) of Included and Parent Studies}




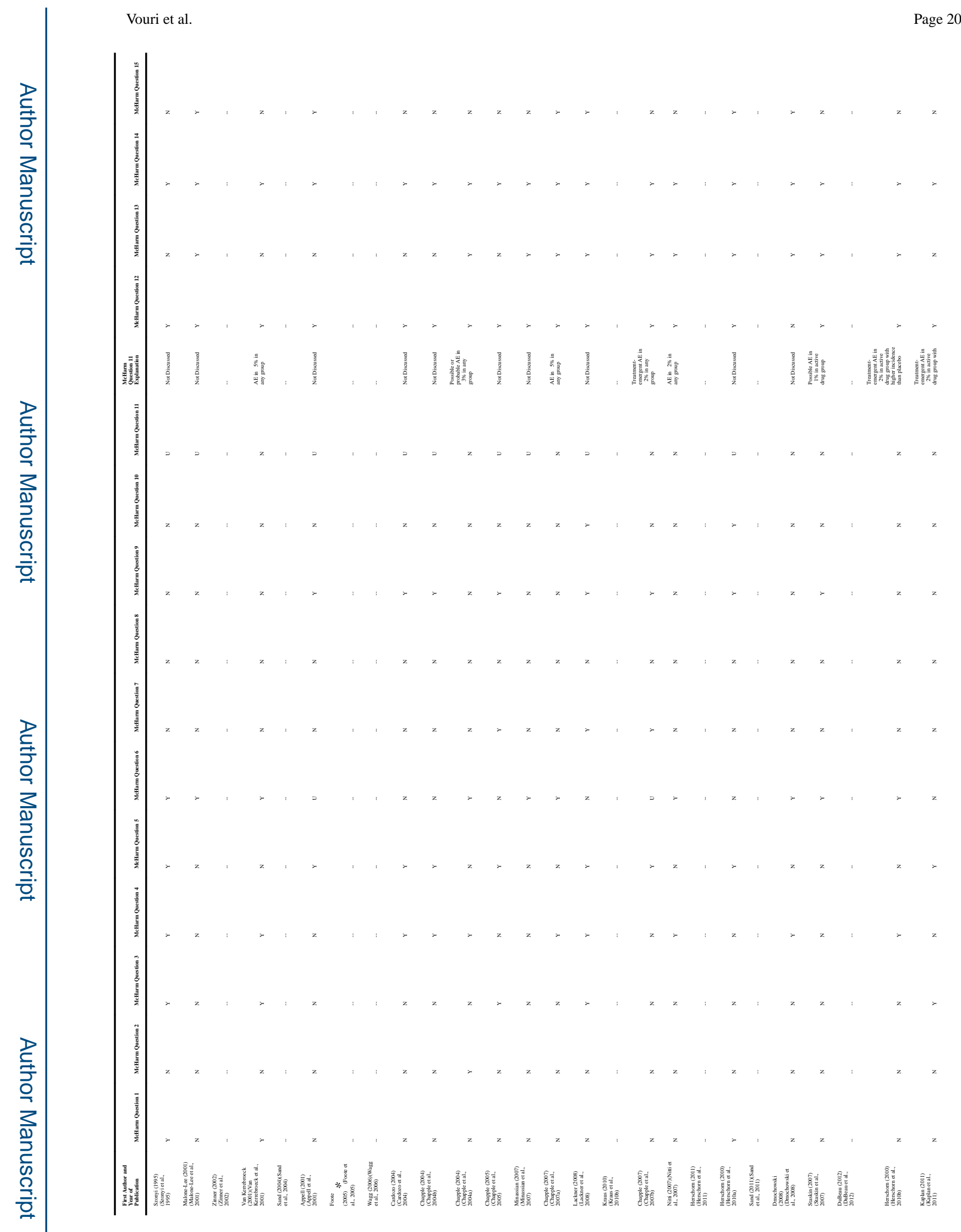

Arch Gerontol Geriatr. Author manuscript; available in PMC 2018 March 01. 


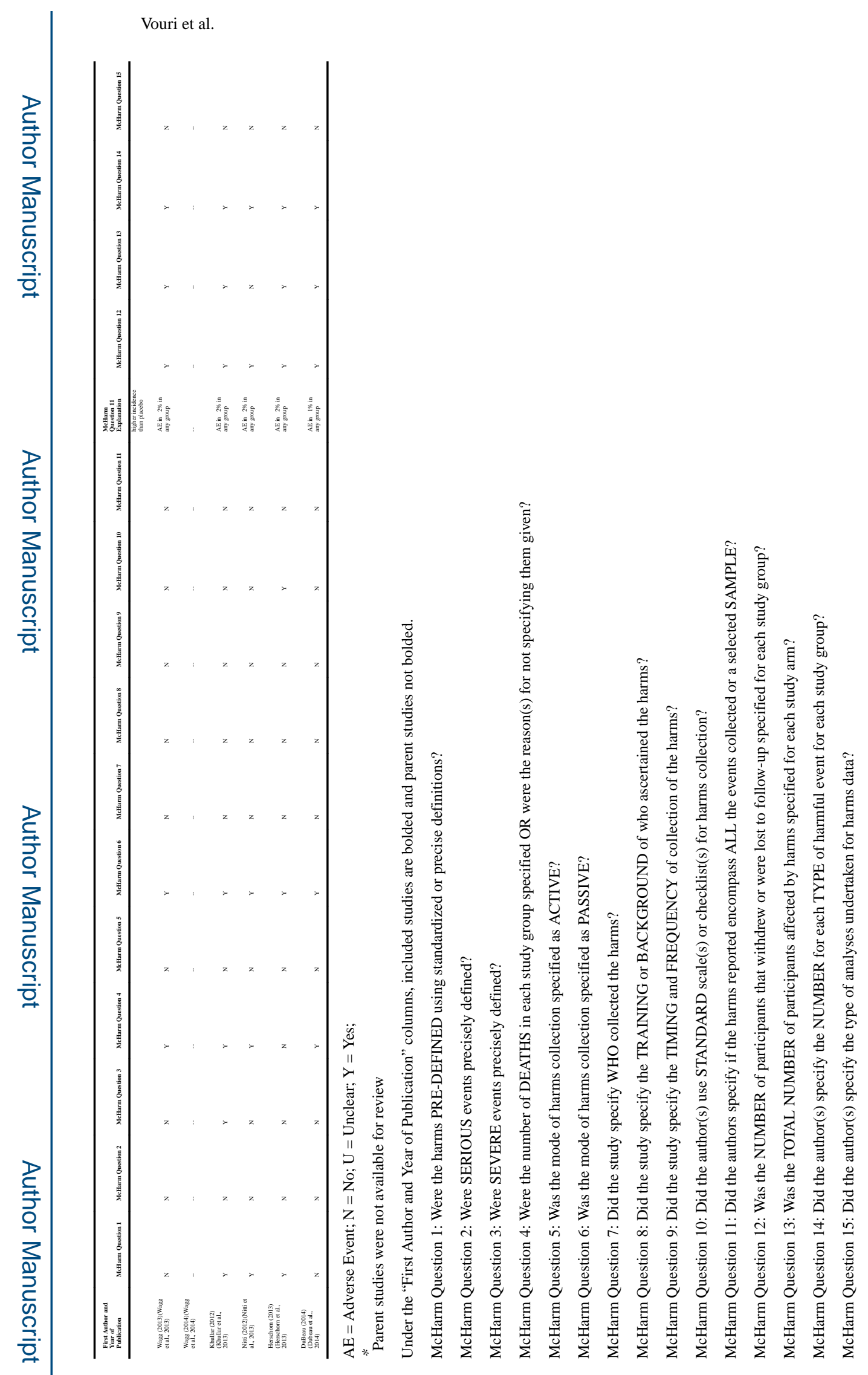

Arch Gerontol Geriatr. Author manuscript; available in PMC 2018 March 01. 


\section{Appendix 3: Adverse Events - Antimuscarinic versus Placebo}




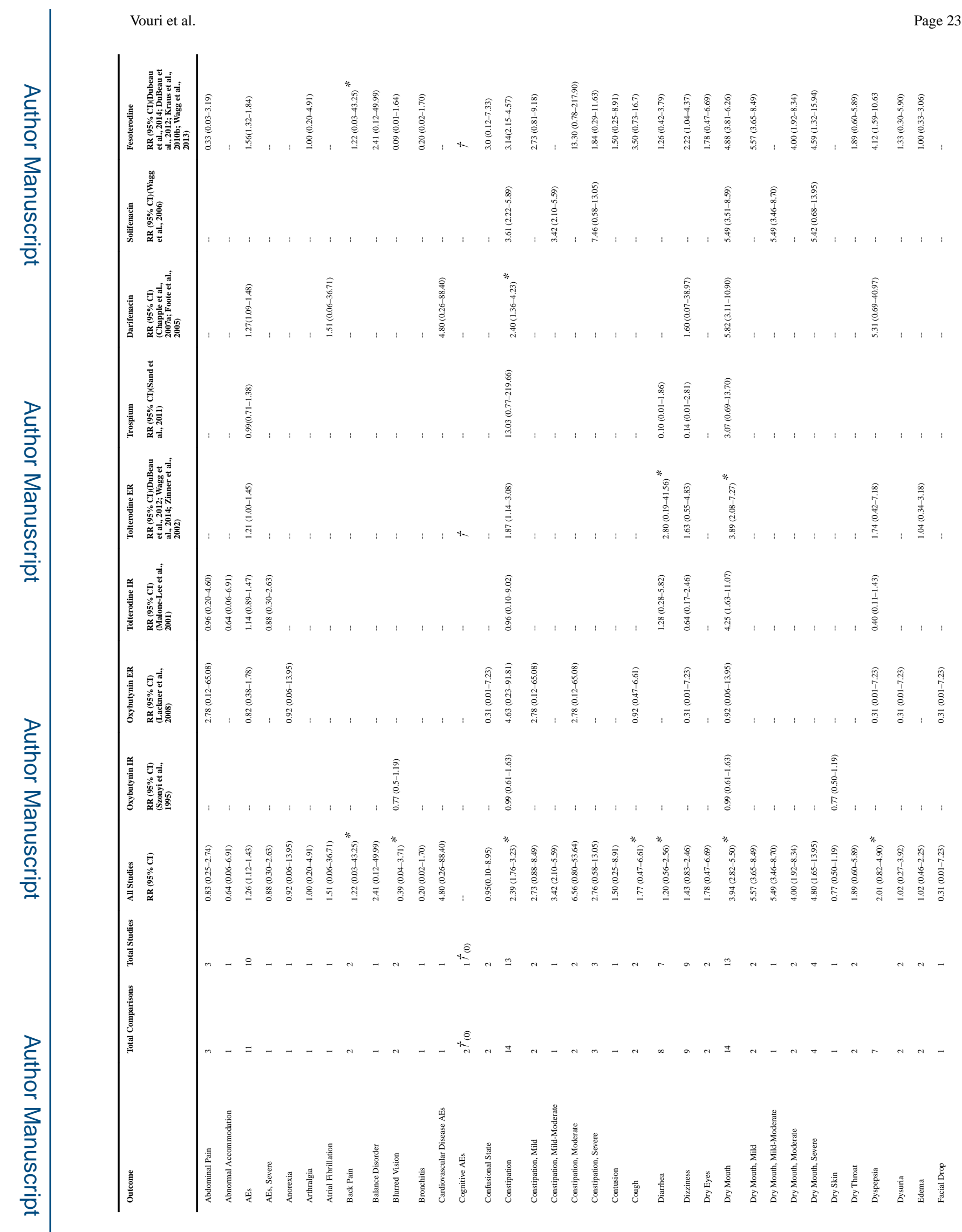

Arch Gerontol Geriatr. Author manuscript; available in PMC 2018 March 01. 


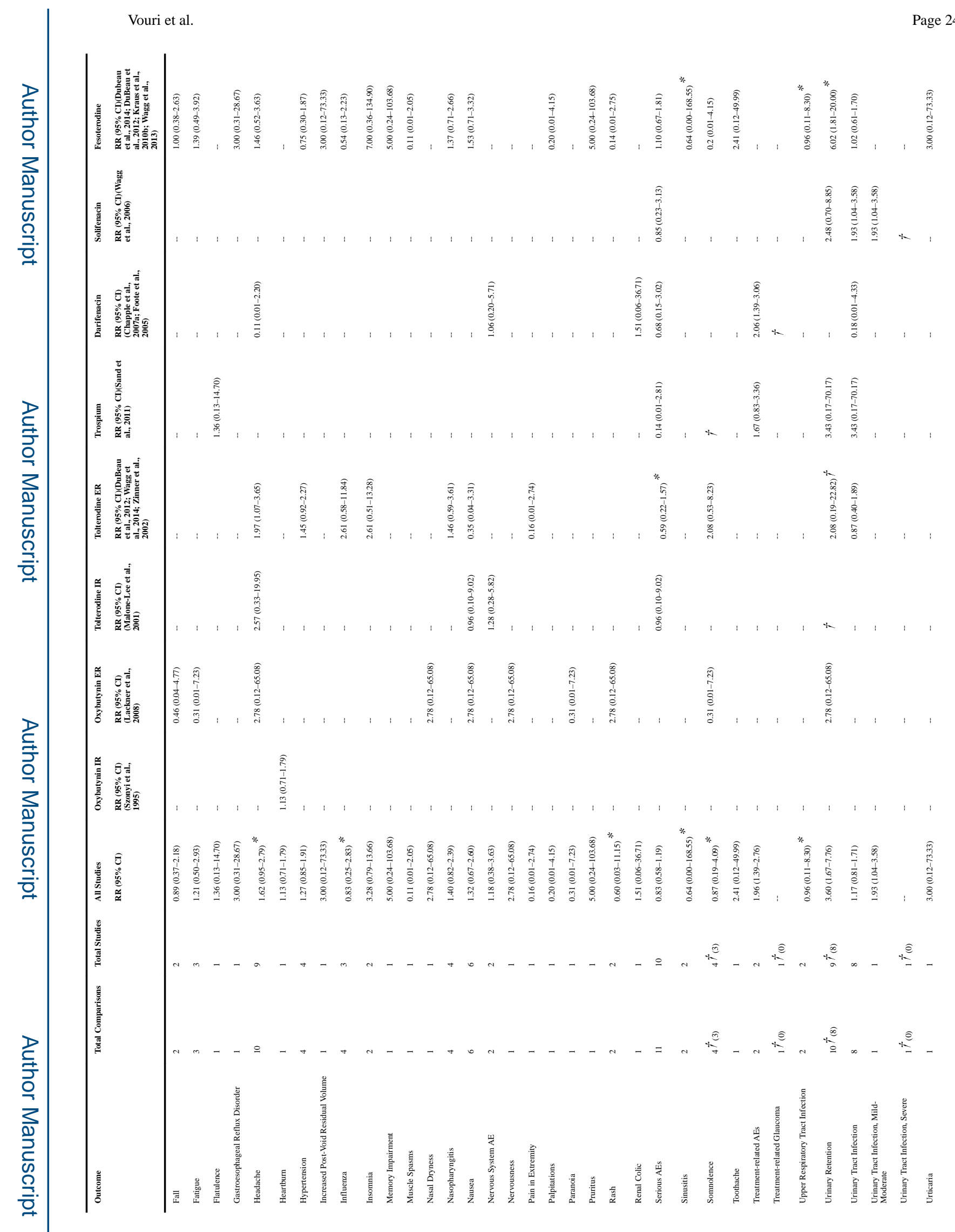

Arch Gerontol Geriatr. Author manuscript; available in PMC 2018 March 01. 


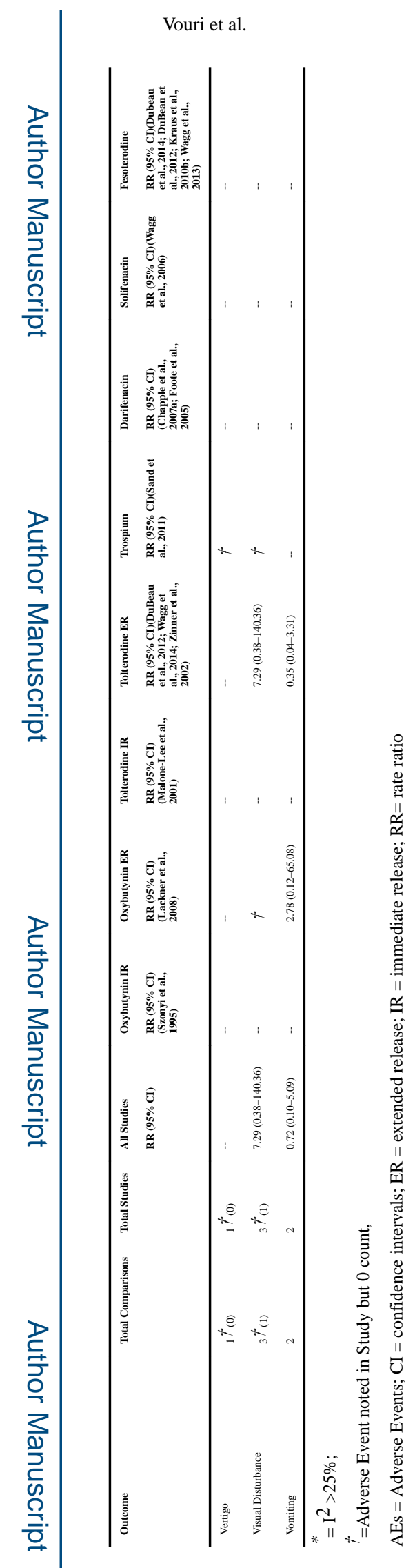

Page 25 
Appendix 4. Adverse Events - Antimuscarinic versus Tolterodine IR/ER or Oxybutynin IR 


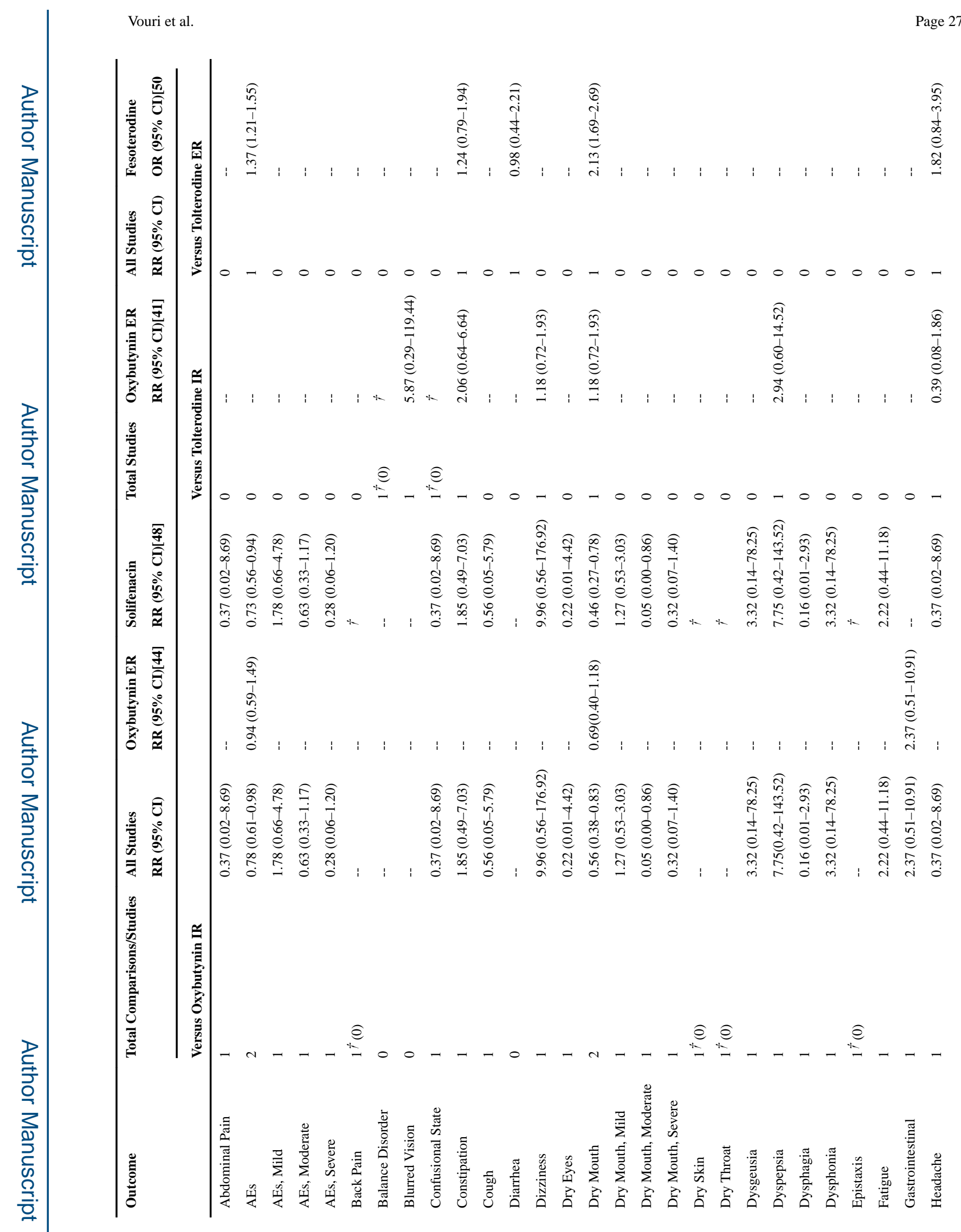

Arch Gerontol Geriatr. Author manuscript; available in PMC 2018 March 01. 


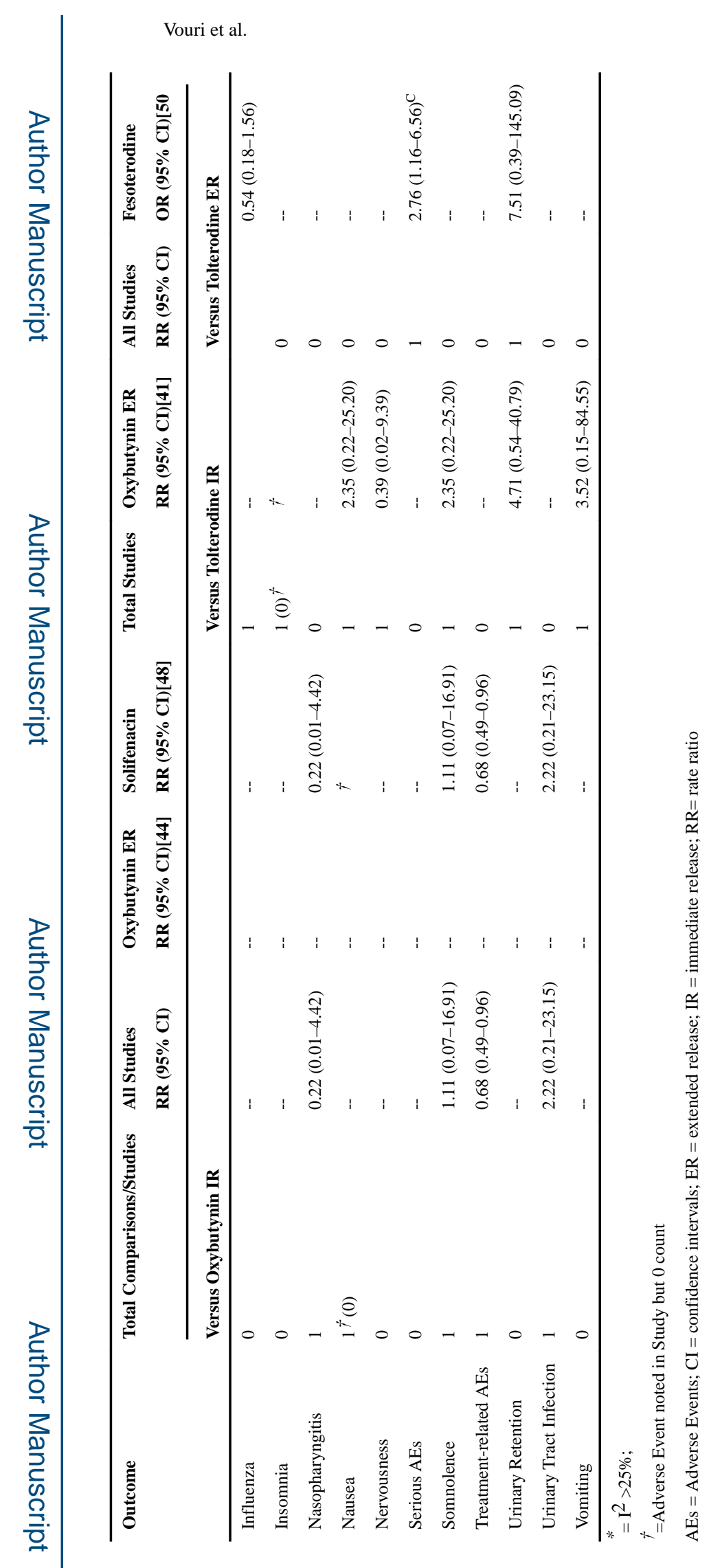

Arch Gerontol Geriatr. Author manuscript; available in PMC 2018 March 01. 
Appendix 5: Reasons for Treatment Discontinuation - Antimuscarinic versus Placebo 


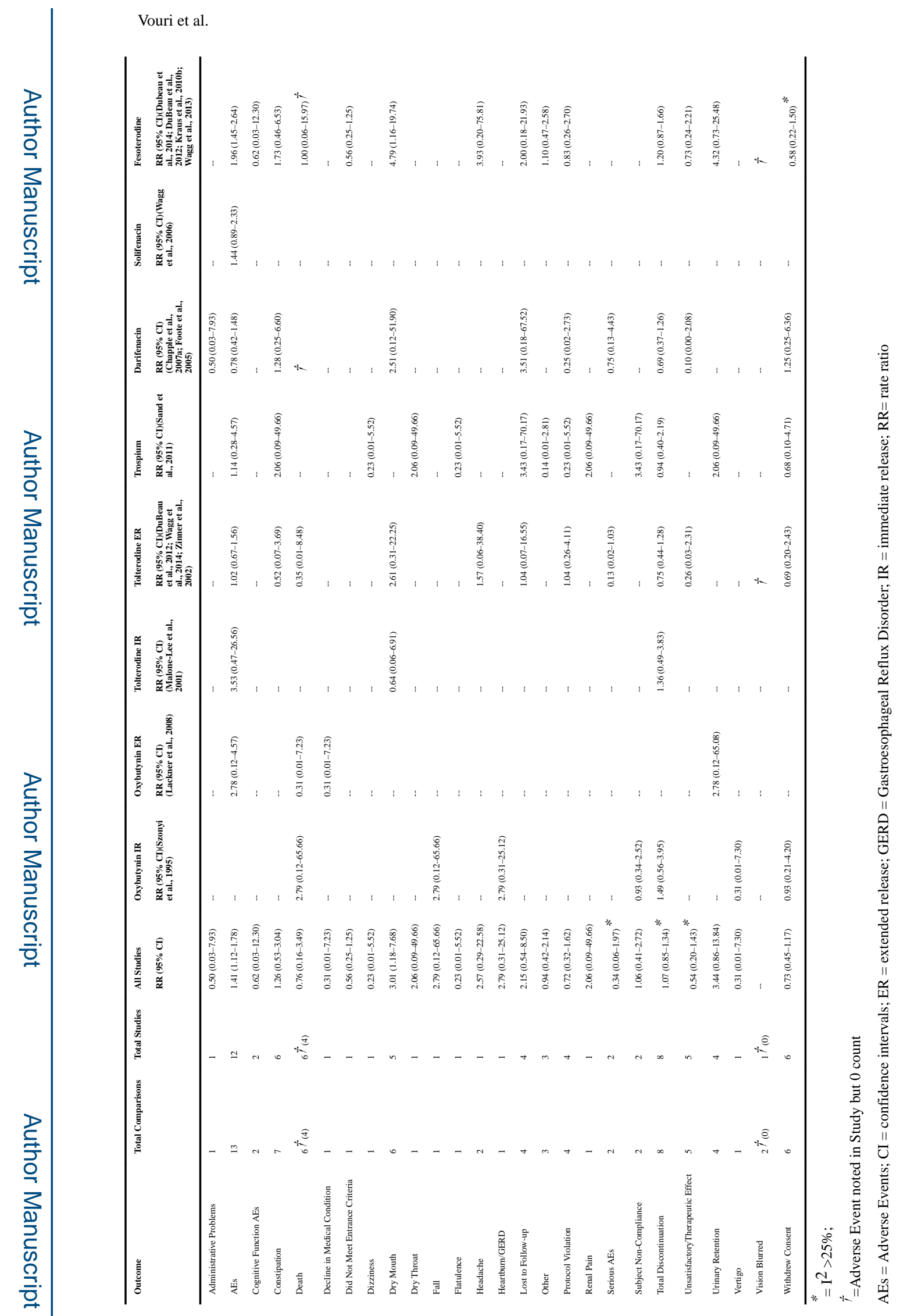

Arch Gerontol Geriatr. Author manuscript; available in PMC 2018 March 01. 
Appendix 6. Reasons for Treatment Discontinuation - Antimuscarinic versus Tolterodine IR/ER or Oxybutynin IR 


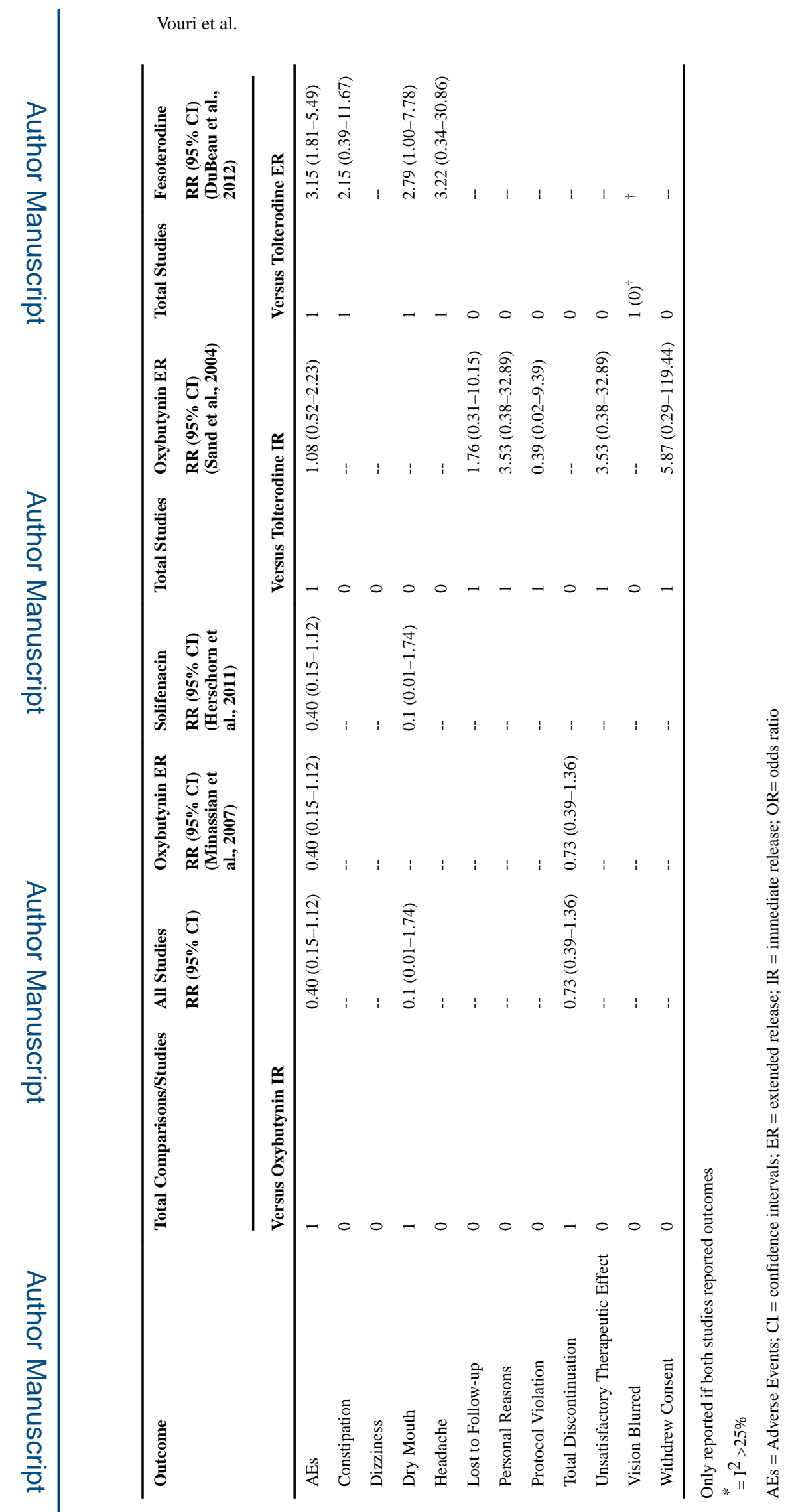

Arch Gerontol Geriatr. Author manuscript; available in PMC 2018 March 01. 
Appendix 7. Combined Adverse Events - Antimuscarinic versus Placebo 


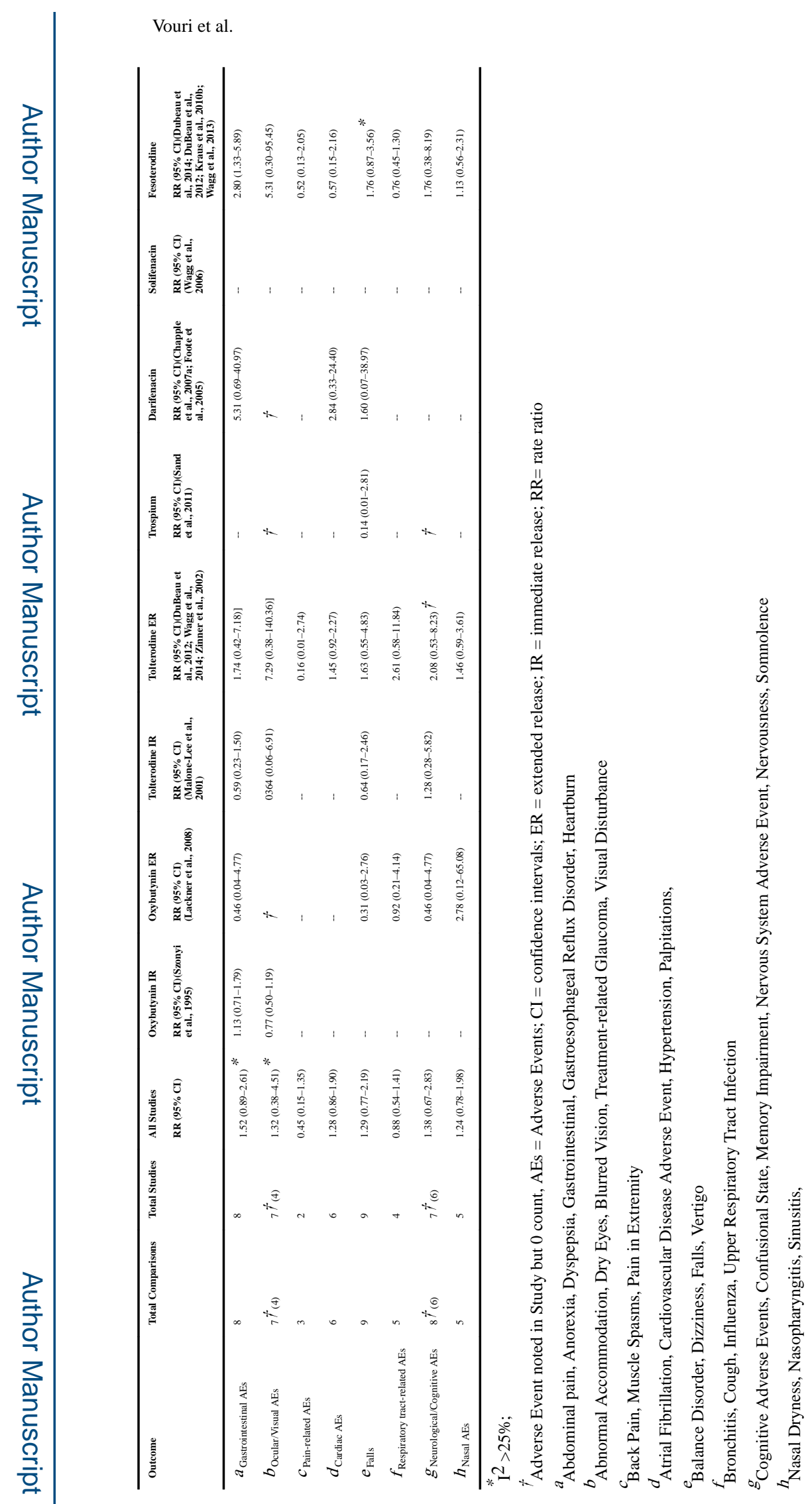


Appendix 8: Combined Adverse Events - Antimuscarinic versus Tolterodine IR/ER or Oxybutynin IR 


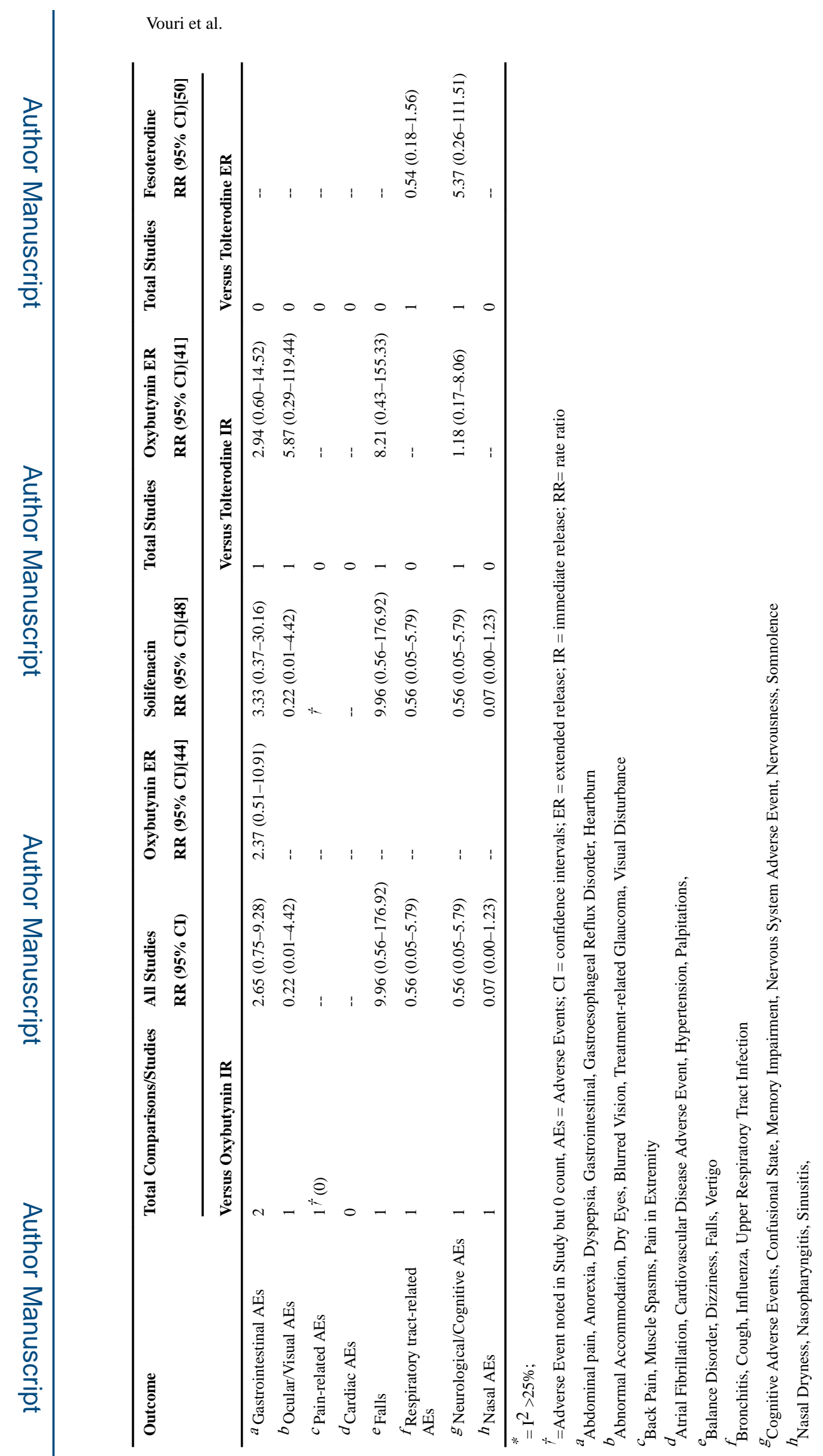

Arch Gerontol Geriatr. Author manuscript; available in PMC 2018 March 01. 


\section{Appendix 9: Forest Plots for All Analysis}

Appendix 9.001: Adnominal Pain (Antimuscarinics versus Placebo)

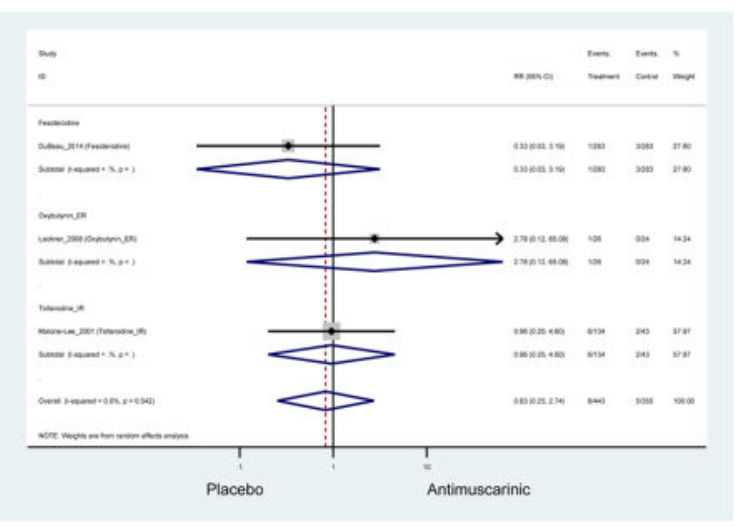

Appendix 9.002: Abnormal Accommodation (Antimuscarinics versus Placebo)

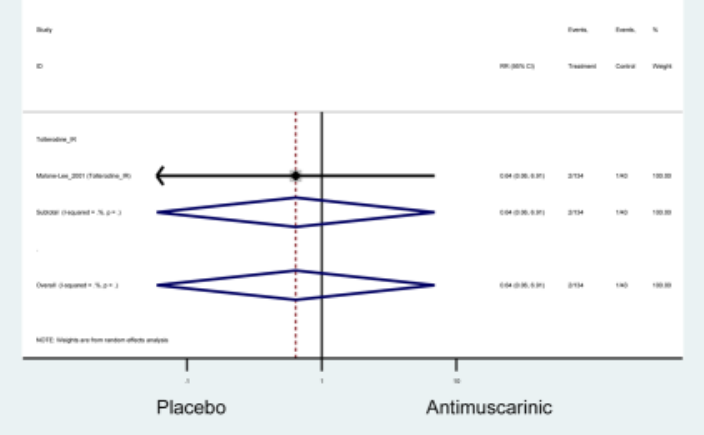

Appendix 9.003: Adverse Events (Antimuscarinics versus Placebo)

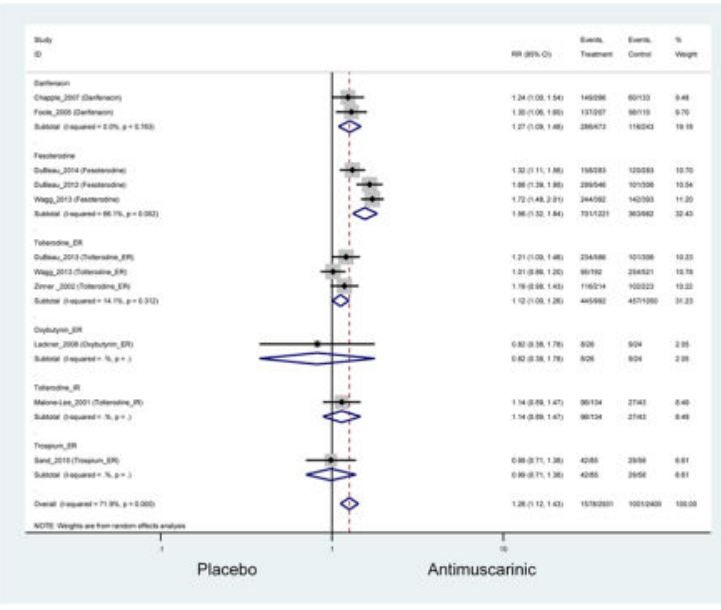

Appendix 9.004: Adverse Events, Severe (Antimuscarinics versus Placebo) 


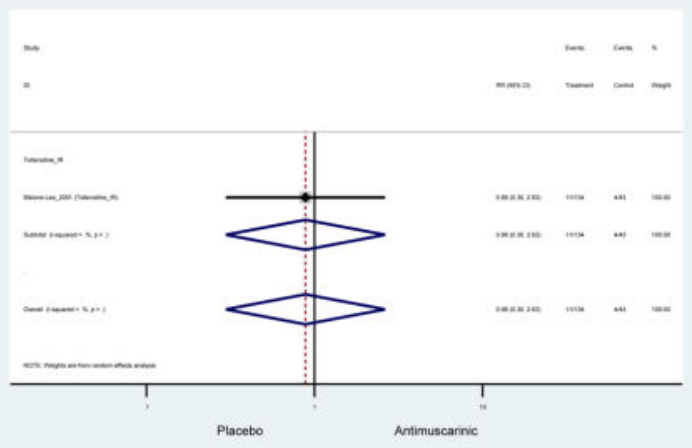

Appendix 9.005: Anorexia (Antimuscarinics versus Placebo)

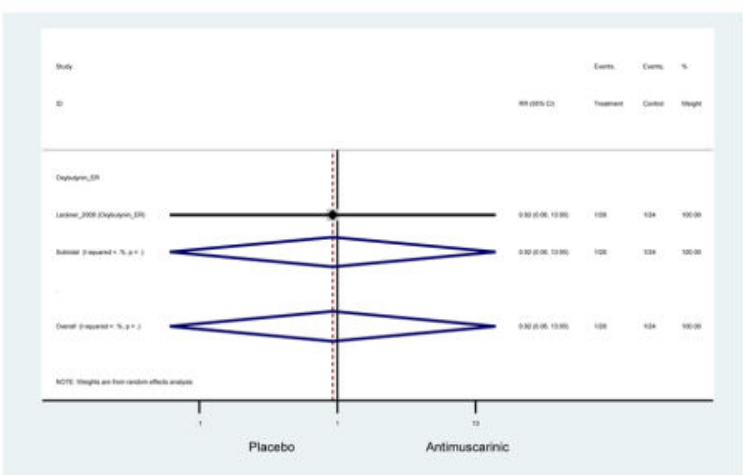

Appendix 9.006: Arthralgia (Antimuscarinics versus Placebo)

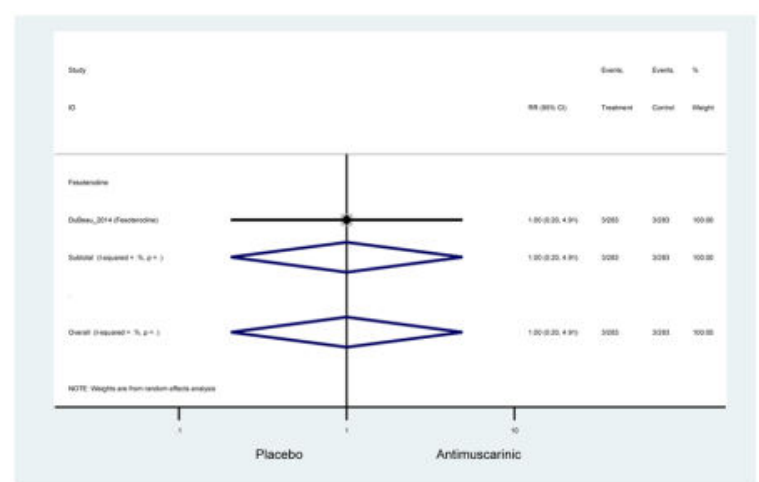

Appendix 9.007: Atrial Fibrillation (Antimuscarinics versus Placebo) 


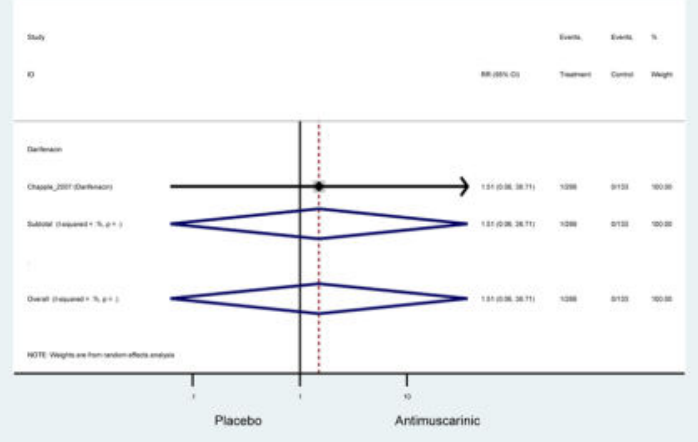

Appendix 9.008: Back Pain (Antimuscarinics versus Placebo)

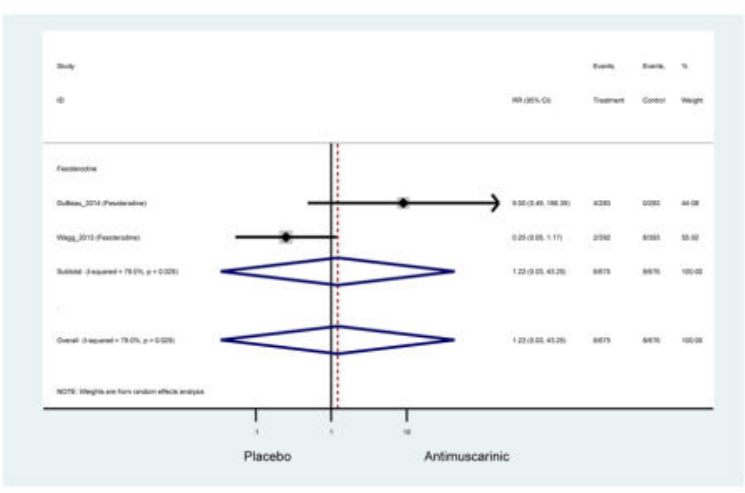

Appendix 9.009: Balance Disorder (Antimuscarinics versus Placebo)

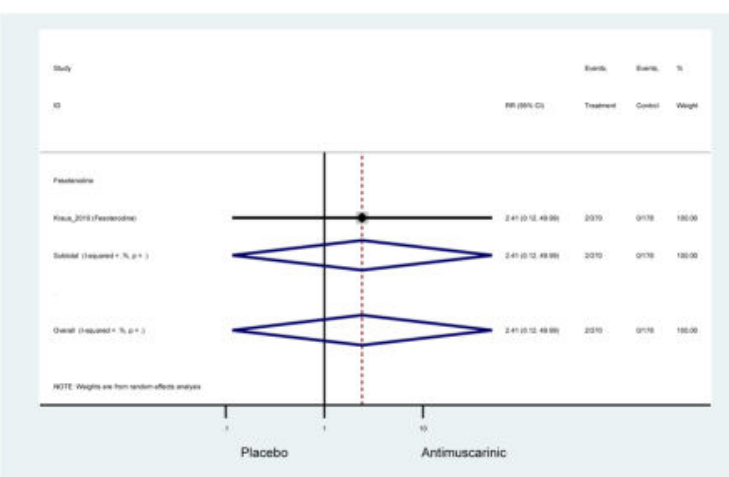

Appendix 9.010: Blurred Vision (Antimuscarinics versus Placebo) 


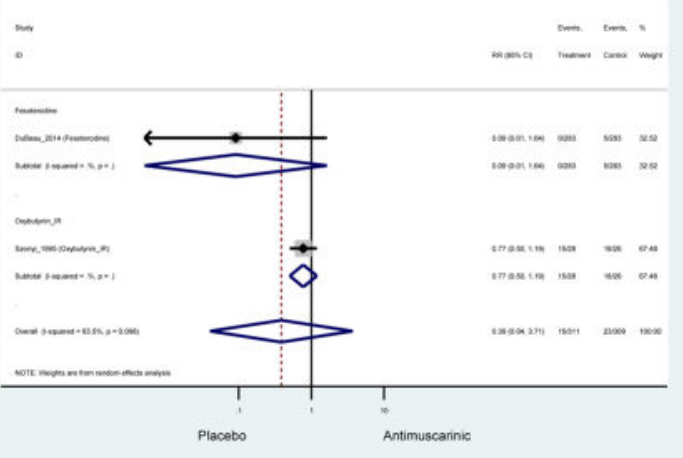

Appendix 9.011: Bronchitis (Antimuscarinics versus Placebo)

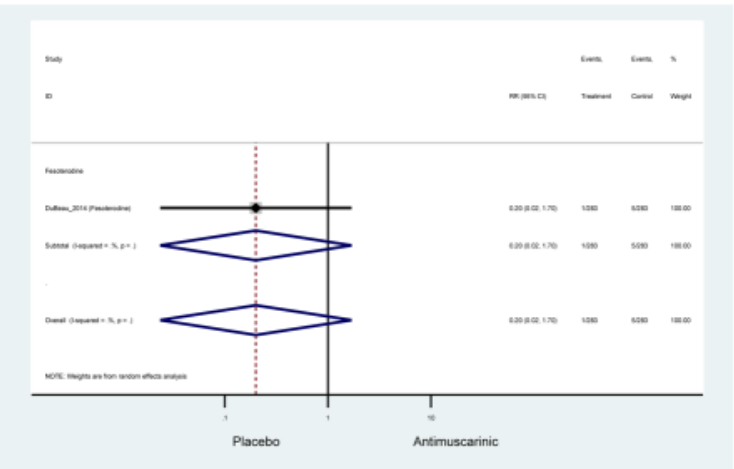

Appendix 9.012: Cardiovascular Disease Adverse Event (Antimuscarinics versus Placebo)

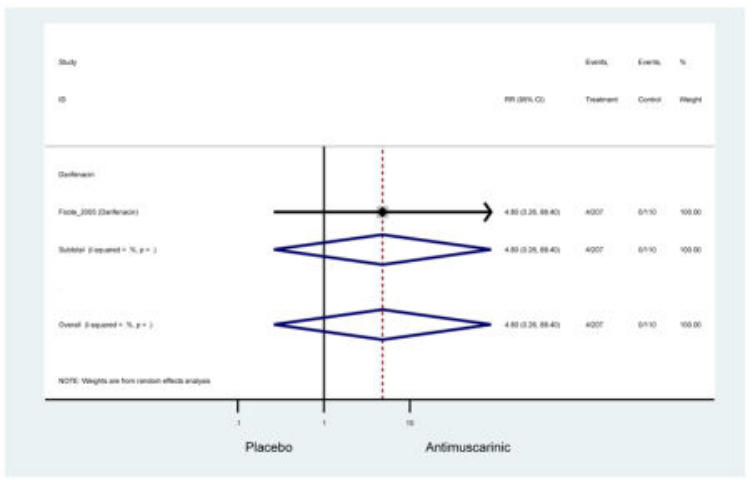

Appendix 9.013: Cognitive Adverse Events (Antimuscarinics versus Placebo)

Zero Outcomes

Appendix 9.014: Confusional State (Antimuscarinics versus Placebo) 


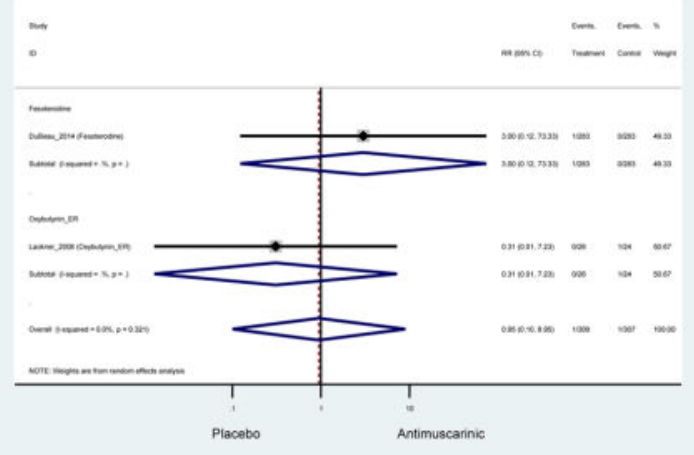

Appendix 9.015: Constipation (Antimuscarinics versus Placebo)

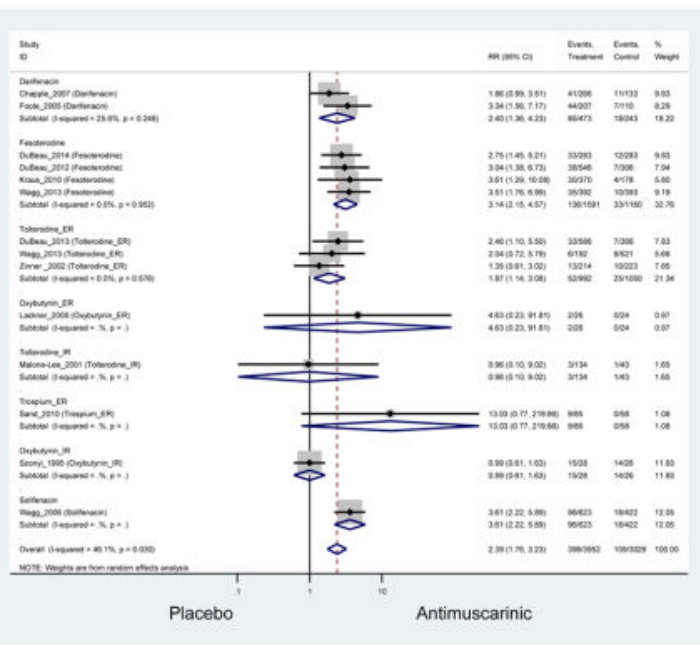

Appendix 9.016: Constipation, Mild (Antimuscarinics versus Placebo)

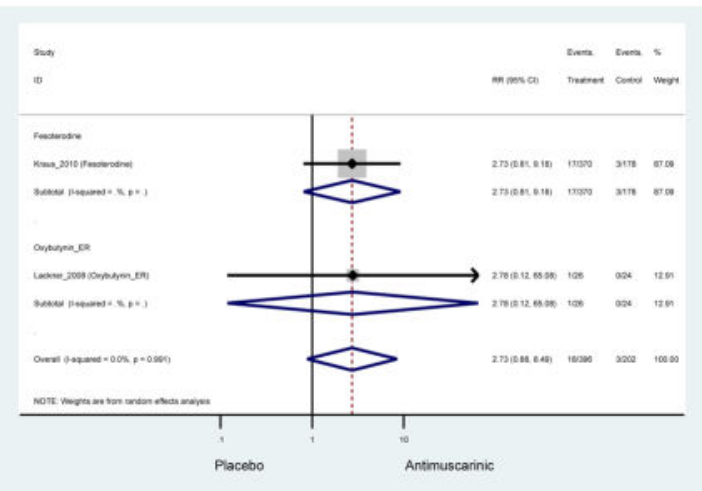

Appendix 9.017: Constipation, Mild-Moderate (Antimuscarinics versus Placebo)

Arch Gerontol Geriatr. Author manuscript; available in PMC 2018 March 01. 


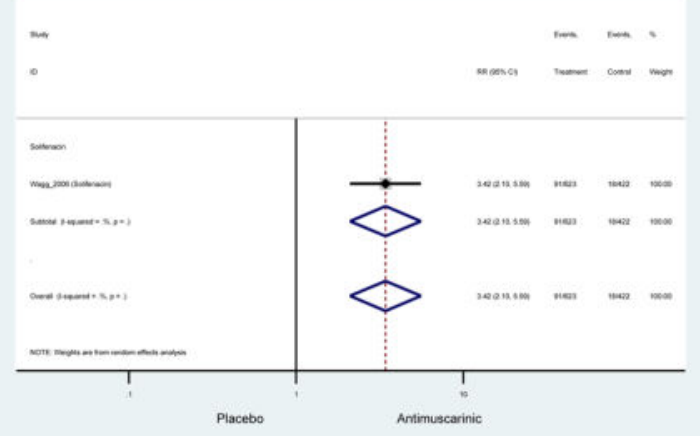

Appendix 9.018: Constipation, Moderate (Antimuscarinics versus Placebo)

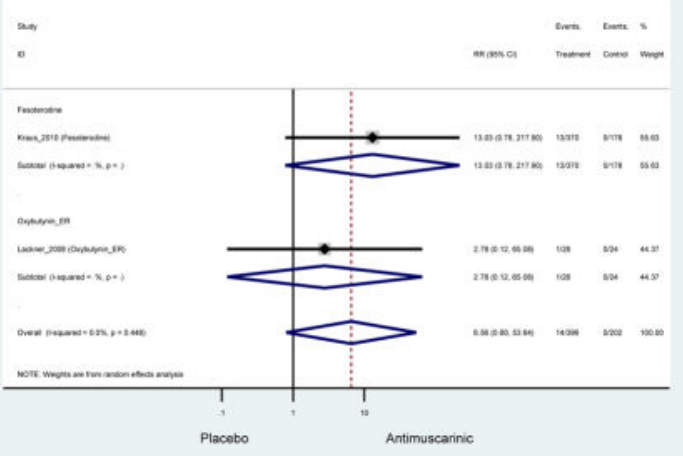

Appendix 9.019: Constipation, Severe (Antimuscarinics versus Placebo)
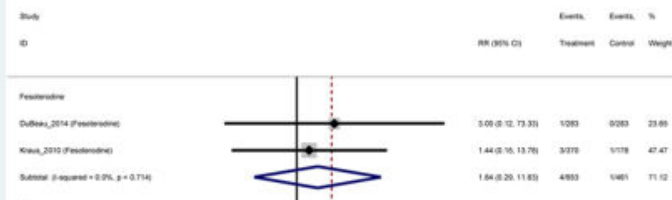

$\sin$

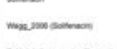
(nis

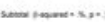

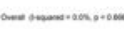
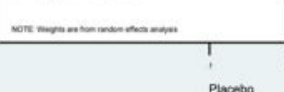

"Animuscarine

Appendix 9.020: Contusion (Antimuscarinics versus Placebo) 


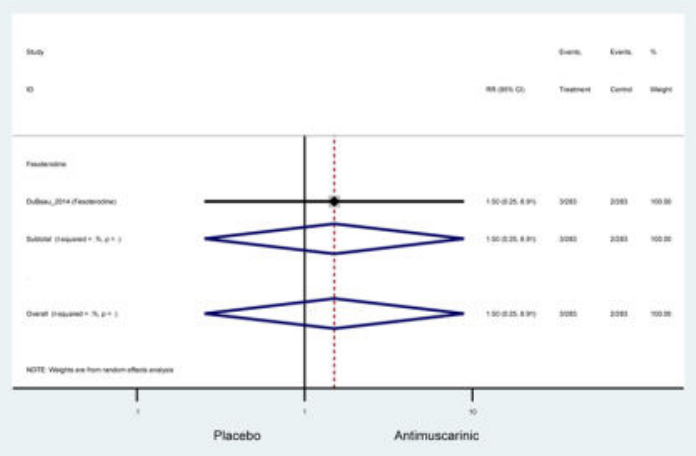

Appendix 9.021: Cough (Antimuscarinics versus Placebo)

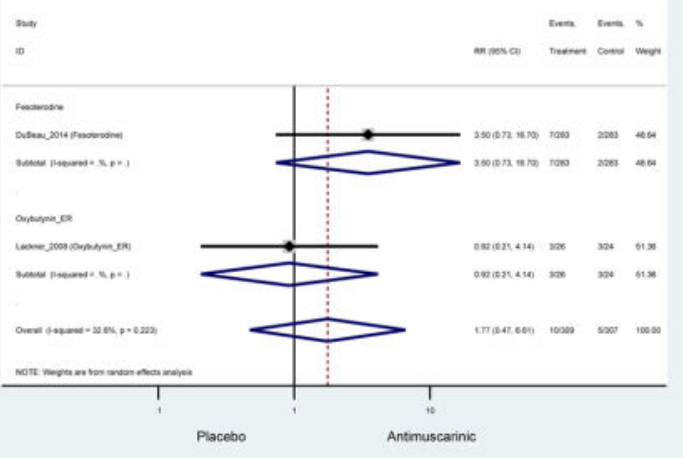

Appendix 9.022: Diarrhea (Antimuscarinics versus Placebo)

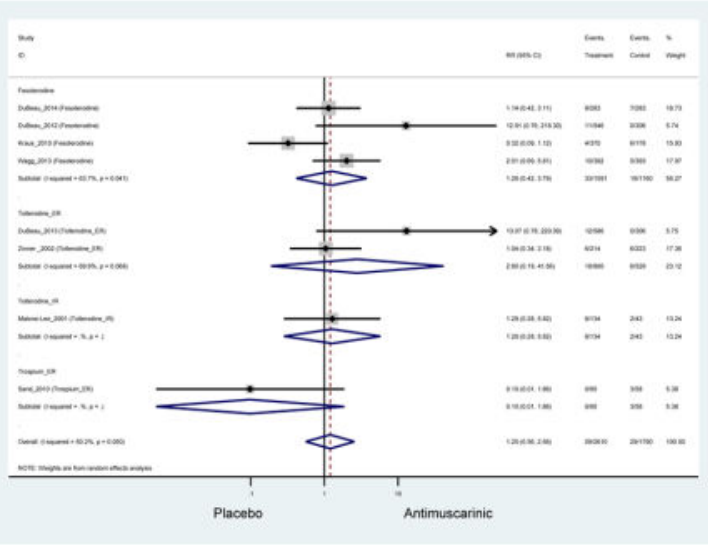

Appendix 9.023: Dizziness (Antimuscarinics versus Placebo)

Arch Gerontol Geriatr. Author manuscript; available in PMC 2018 March 01. 


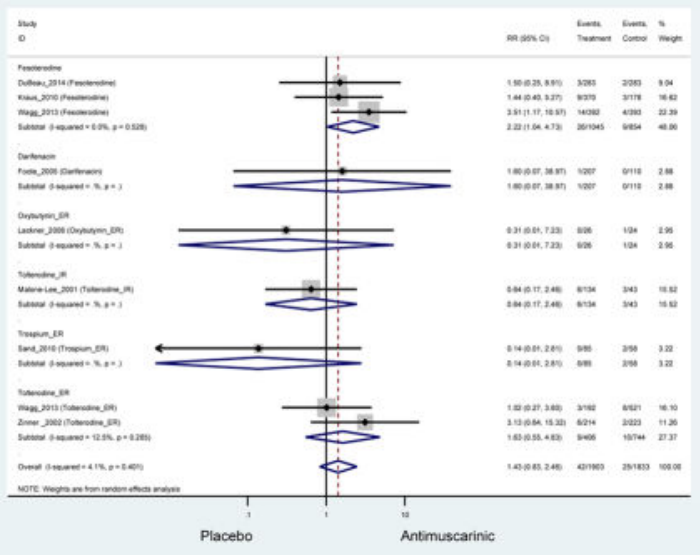

Appendix 9.024: Dry Eyes (Antimuscarinics versus Placebo)

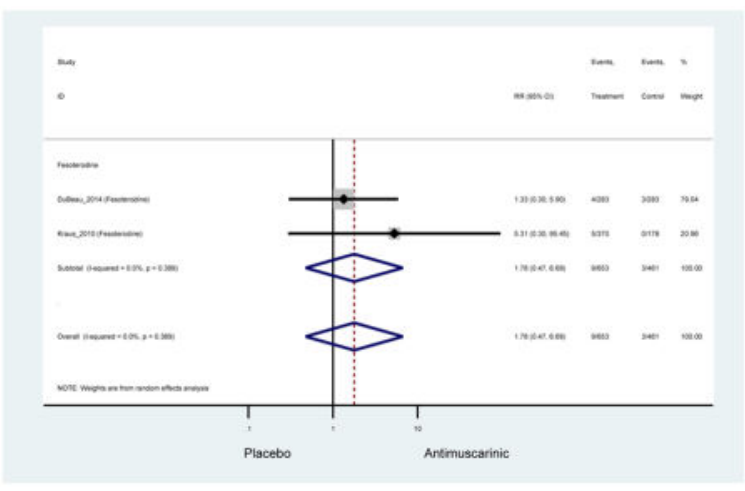

Appendix 9.025: Dry Mouth (Antimuscarinics versus Placebo)

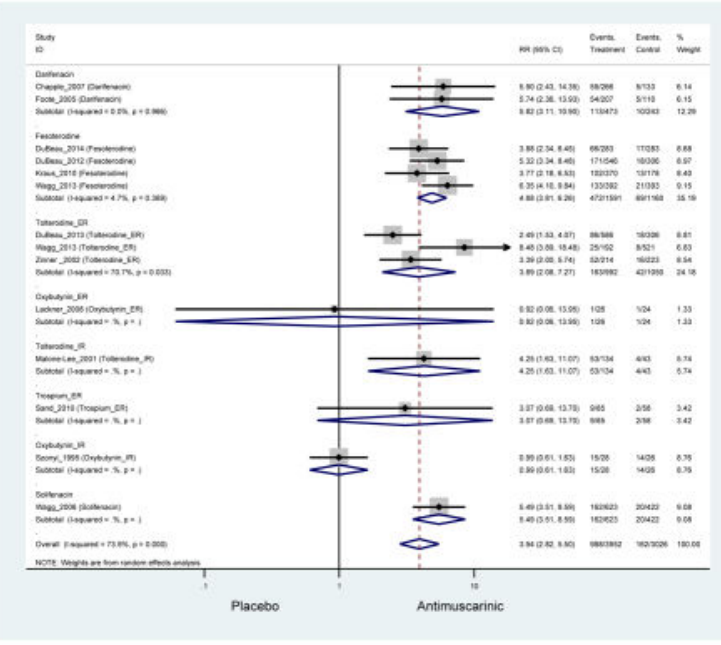

Appendix 9.026: Dry Mouth, Mild (Antimuscarinics versus Placebo)

Arch Gerontol Geriatr. Author manuscript; available in PMC 2018 March 01. 


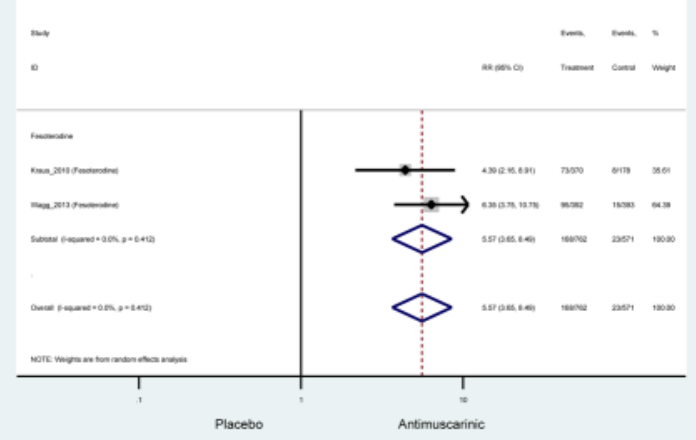

Appendix 9.027: Dry Mouth, Mild-Moderate (Antimuscarinics versus Placebo)
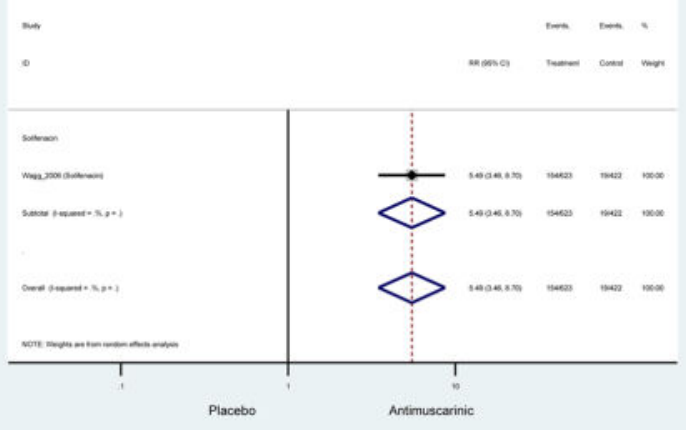

Appendix 9.028: Dry Mouth, Moderate (Antimuscarinics versus Placebo)

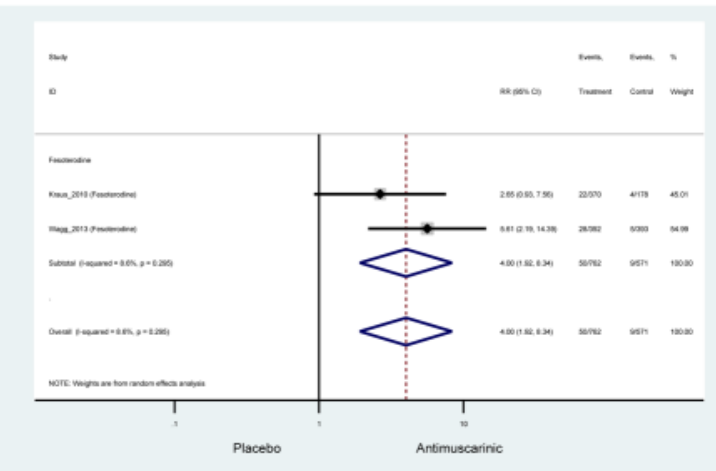

Appendix 9.029: Dry Mouth, Severe (Antimuscarinics versus Placebo) 


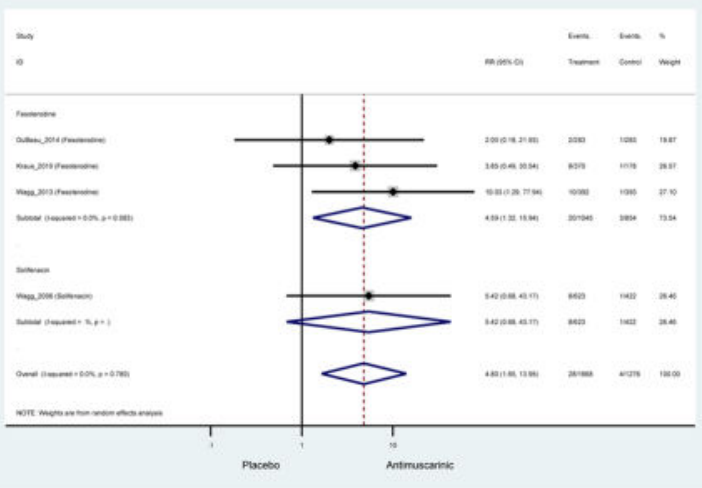

Appendix 9.030: Dry Skin (Antimuscarinics versus Placebo)
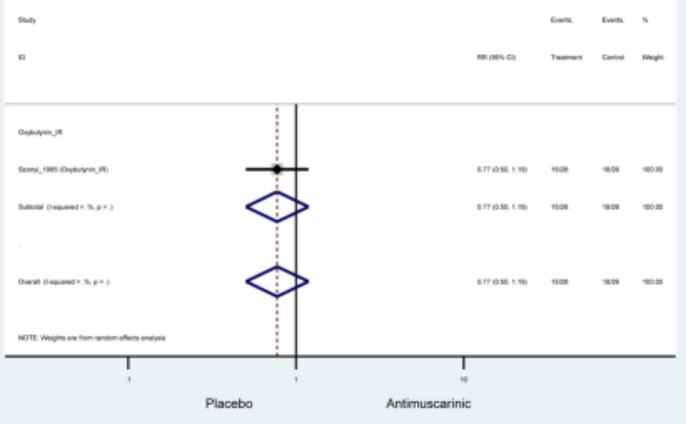

Appendix 9.031: Dry Throat (Antimuscarinics versus Placebo)
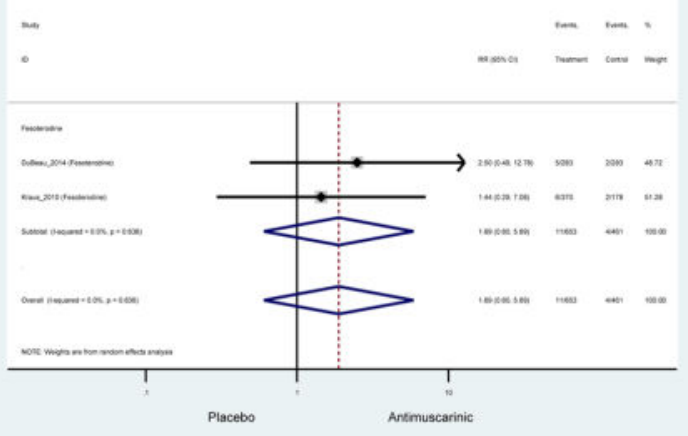

Appendix 9.032: Dyspepsia (Antimuscarinics versus Placebo)

Arch Gerontol Geriatr. Author manuscript; available in PMC 2018 March 01. 


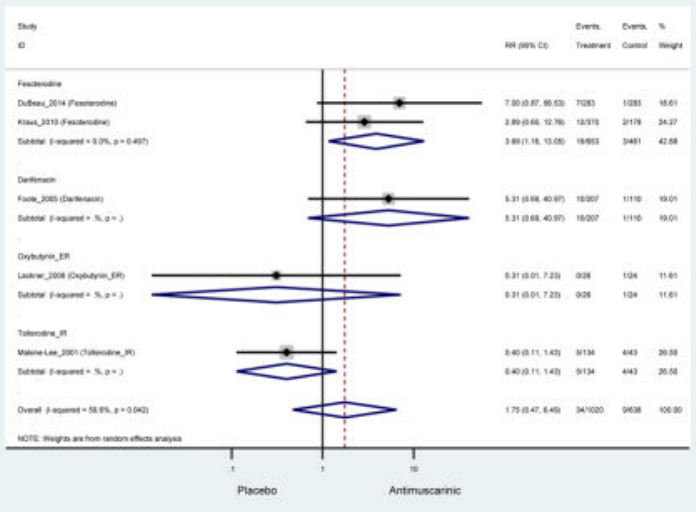

Appendix 9.033: Dysuria (Antimuscarinics versus Placebo)

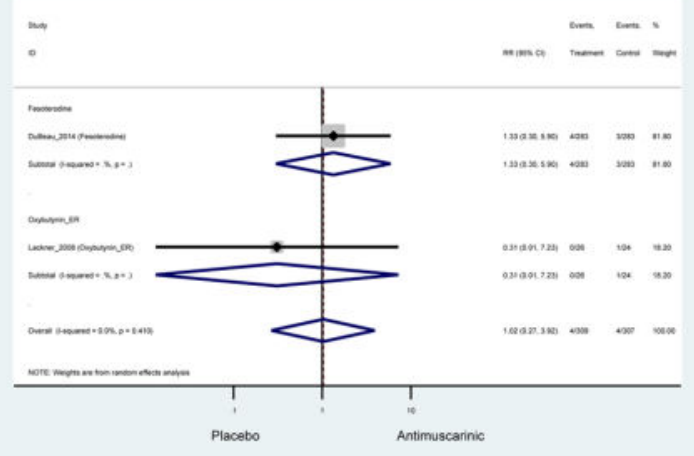

Appendix 9.034: Edema (Antimuscarinics versus Placebo)

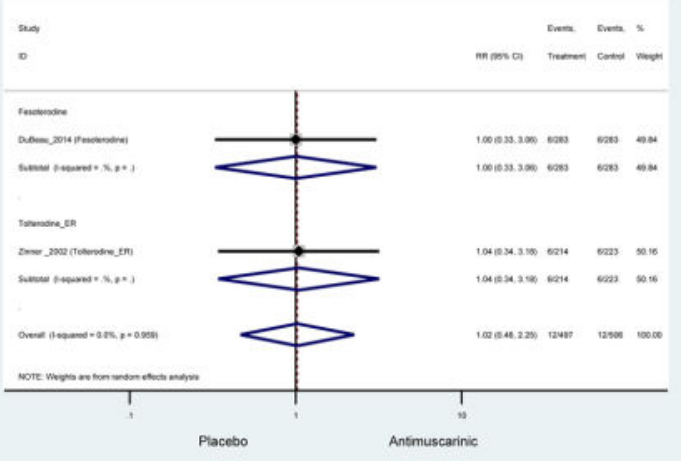

Appendix 9.035: Epistaxis (Antimuscarinics versus Placebo)

Arch Gerontol Geriatr. Author manuscript; available in PMC 2018 March 01. 


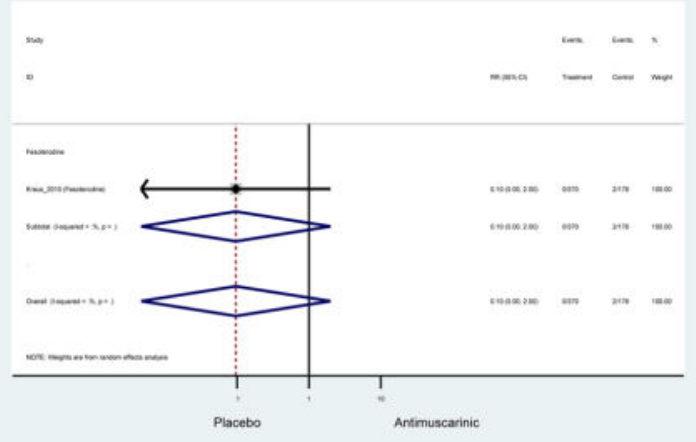

Appendix 9.036: Facial Drop (Antimuscarinics versus Placebo)

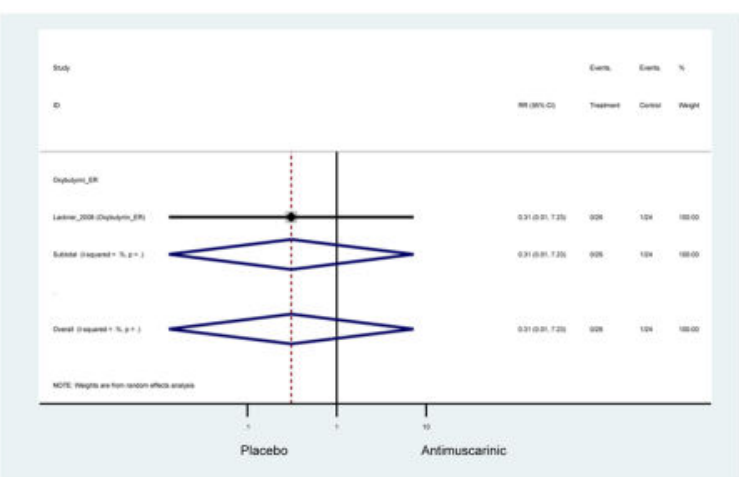

Appendix 9.037: Fall (Antimuscarinics versus Placebo)

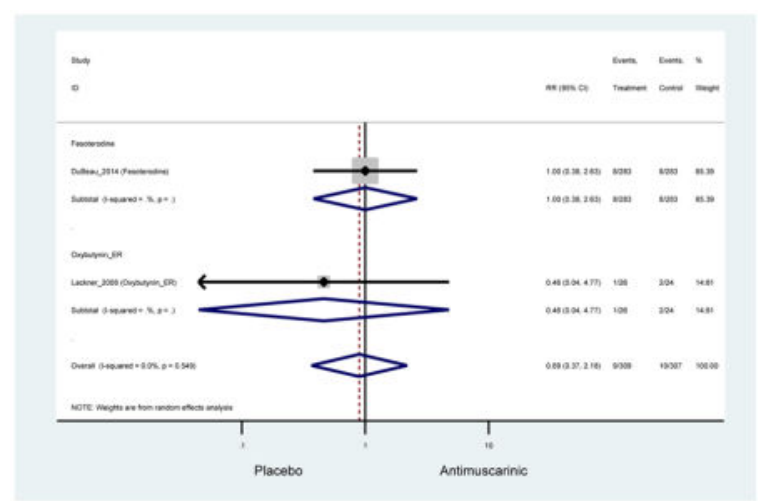

Appendix 9.038: Fatigue (Antimuscarinics versus Placebo) 


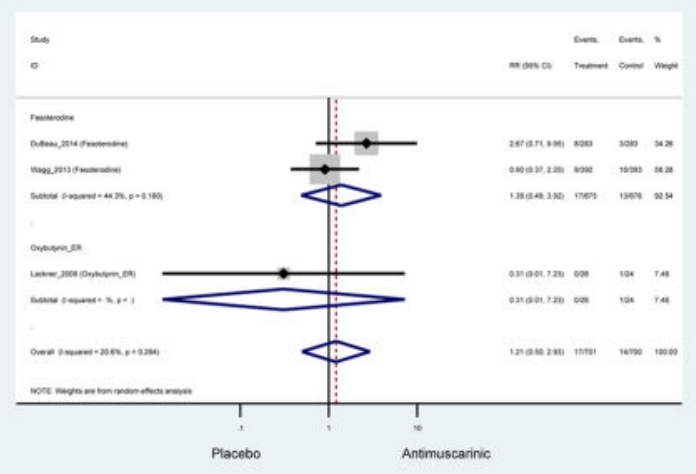

Appendix 9.039: Flatulence (Antimuscarinics versus Placebo)

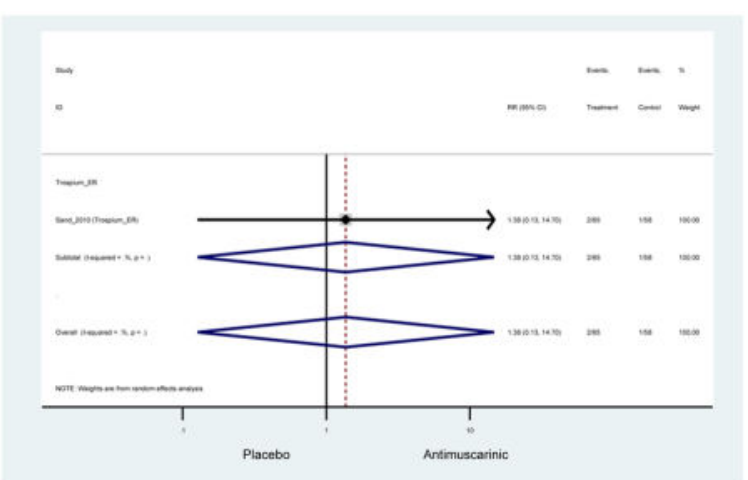

Appendix 9.040: Gastroesophageal Reflux Disorder (Antimuscarinics versus Placebo)

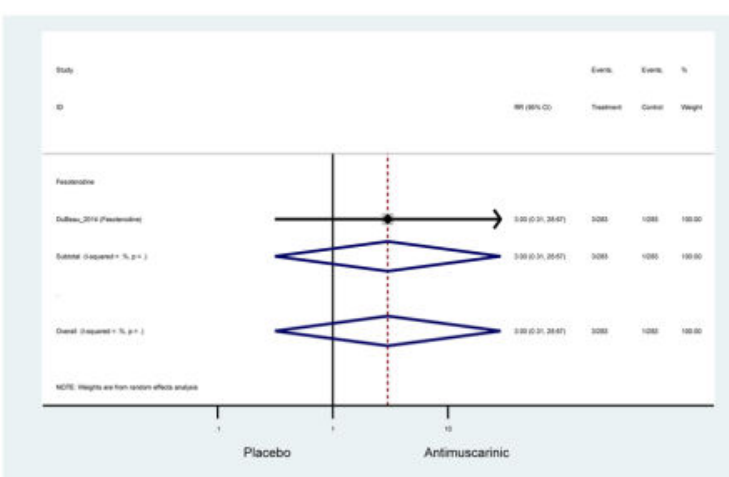

Appendix 9.041: Headache (Antimuscarinics versus Placebo) 


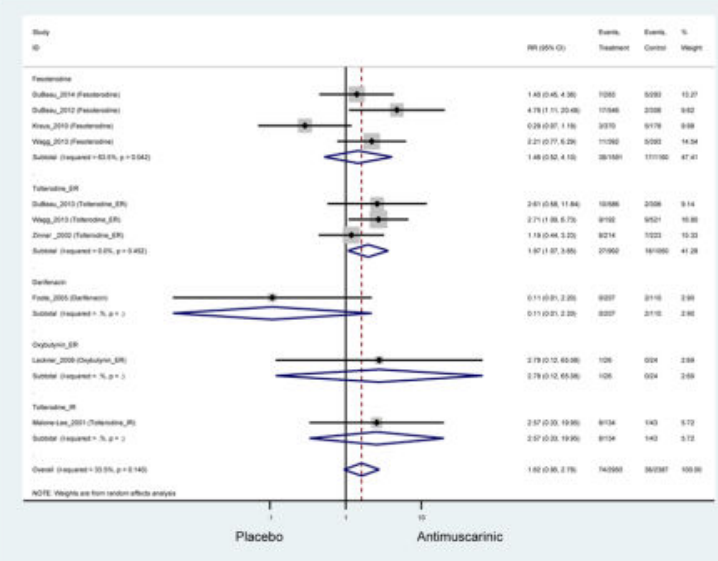

Appendix 9.042: Heartburn (Antimuscarinics versus Placebo)

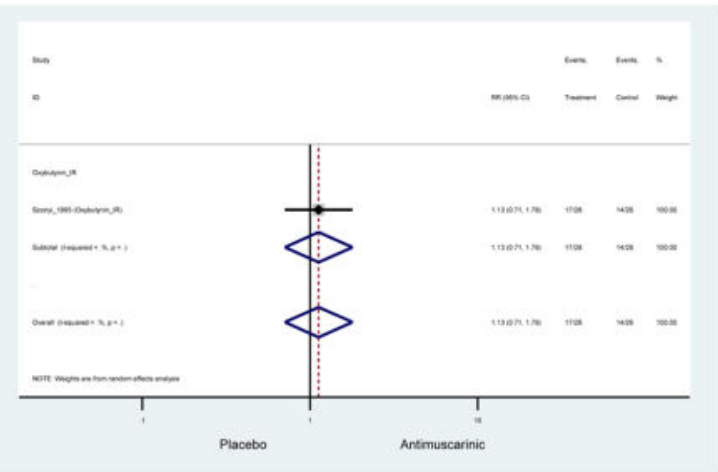

Appendix 9.043: Hypertension (Antimuscarinics versus Placebo)

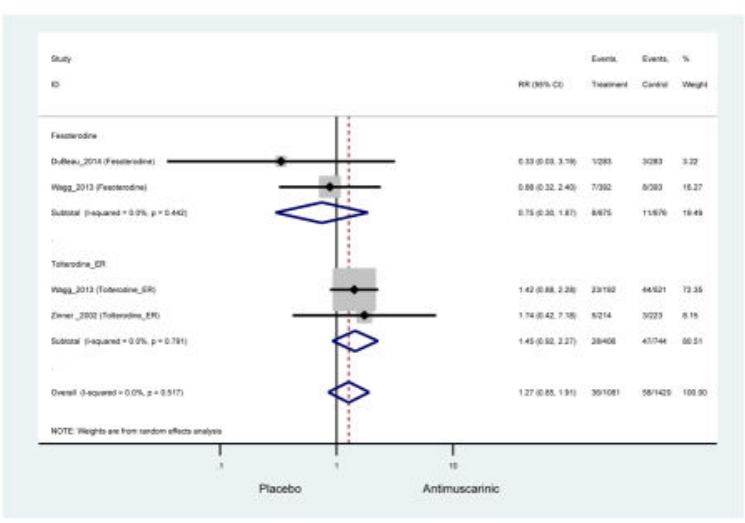

Appendix 9.044: Increased Post-Void Residual Volume (Antimuscarinics versus Placebo) 


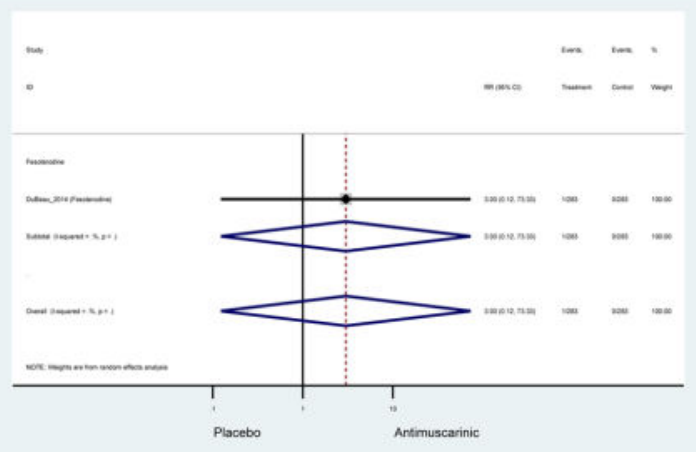

Appendix 9.045: Influenza (Antimuscarinics versus Placebo)

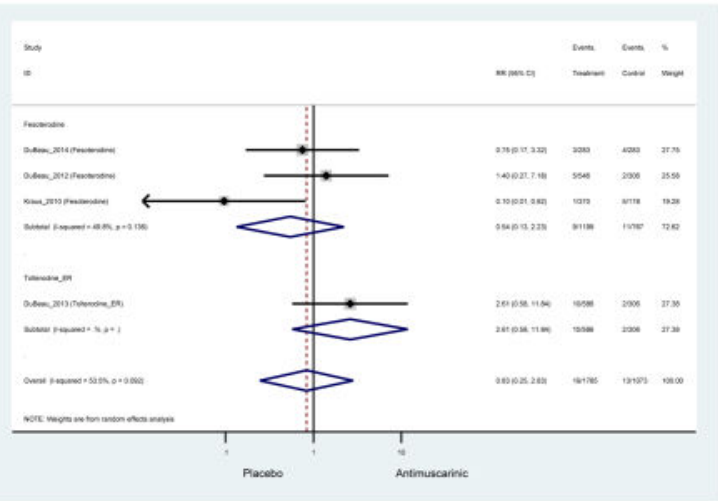

Appendix 9.046: Insomnia (Antimuscarinics versus Placebo)

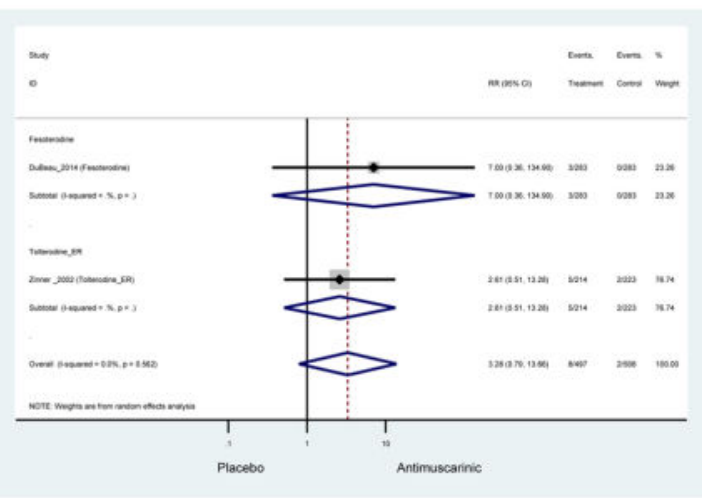

Appendix 9.047: Memory Impairment (Antimuscarinics versus Placebo) 

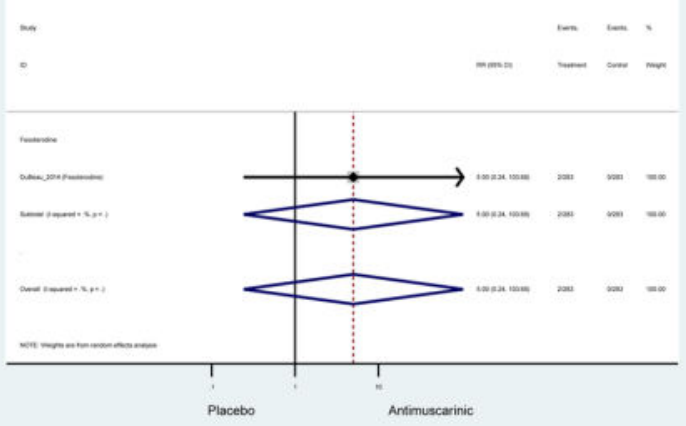

Appendix 9.048: Muscle Spasms (Antimuscarinics versus Placebo)

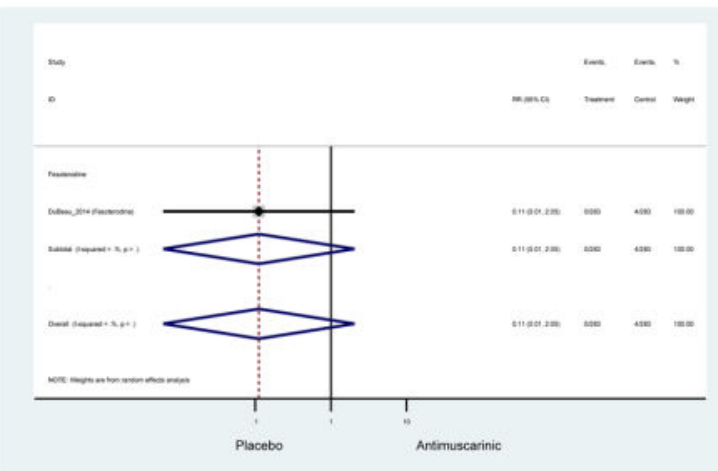

Appendix 9.049: Nasal Dryness (Antimuscarinics versus Placebo)

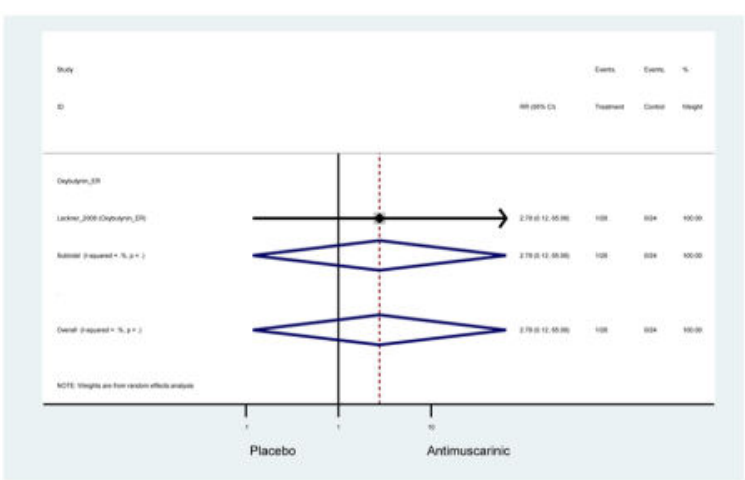

Appendix 9.050: Nasopharyngitis (Antimuscarinics versus Placebo) 


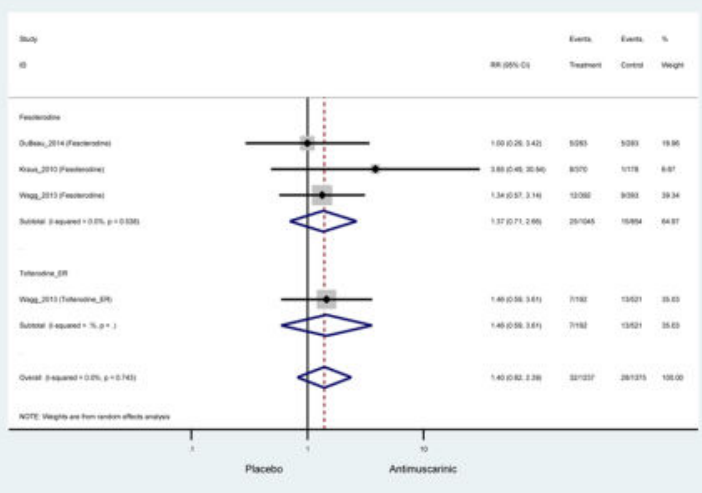

Appendix 9.051: Nausea (Antimuscarinics versus Placebo)

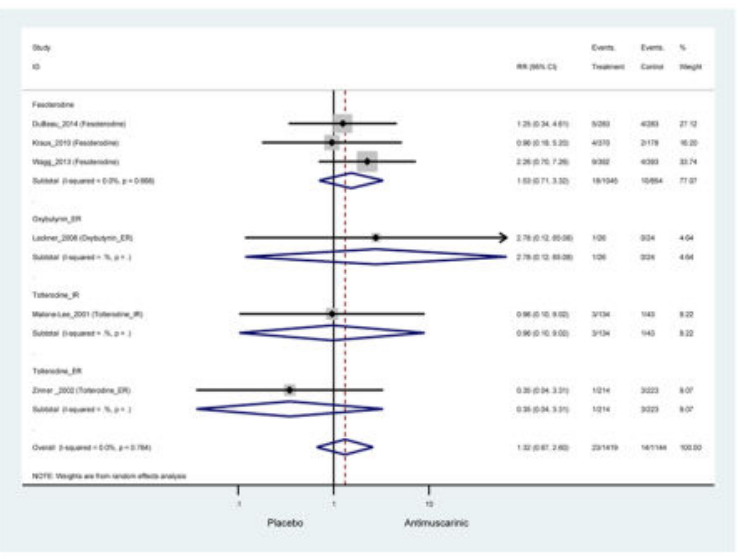

Appendix 9.052: Nervous System Adverse Event (Antimuscarinics versus Placebo)

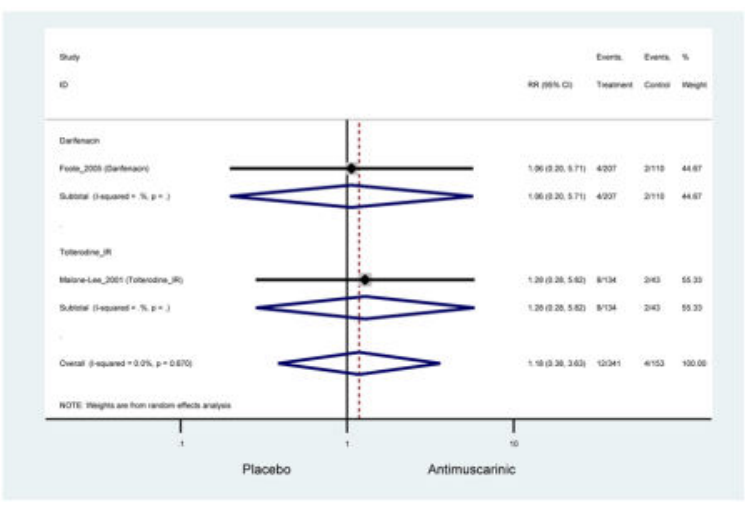

Appendix 9.053: Nervousness (Antimuscarinics versus Placebo) 

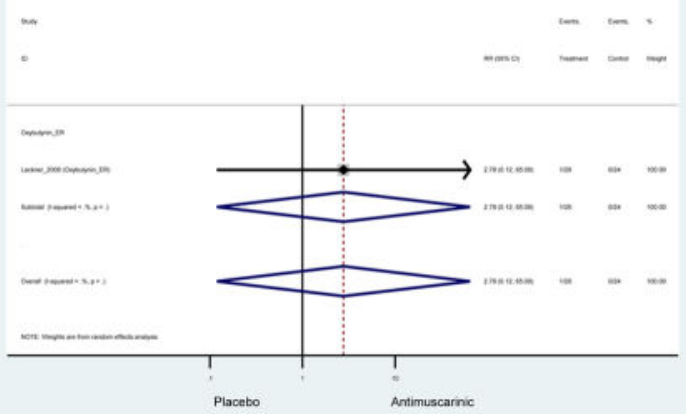

Appendix 9.054: Pain in Extremity (Antimuscarinics versus Placebo)

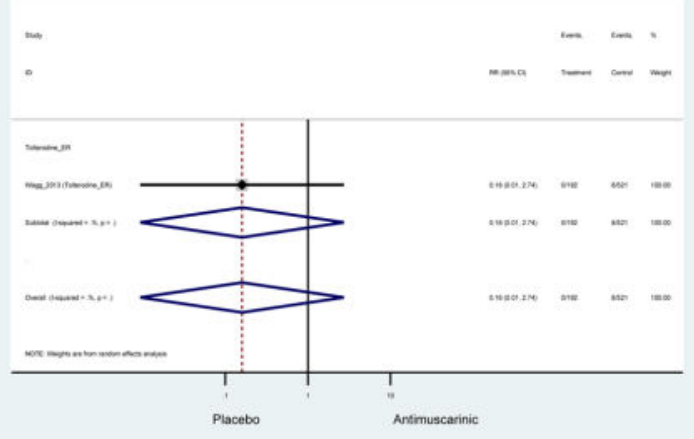

Appendix 9.055: Palpitations (Antimuscarinics versus Placebo)
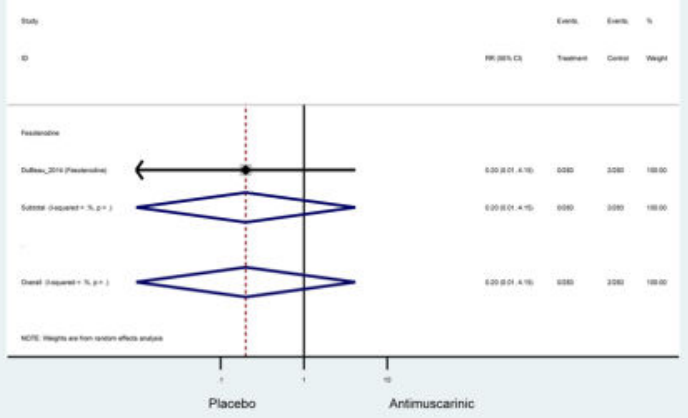

Appendix 9.056: Paranoia (Antimuscarinics versus Placebo) 


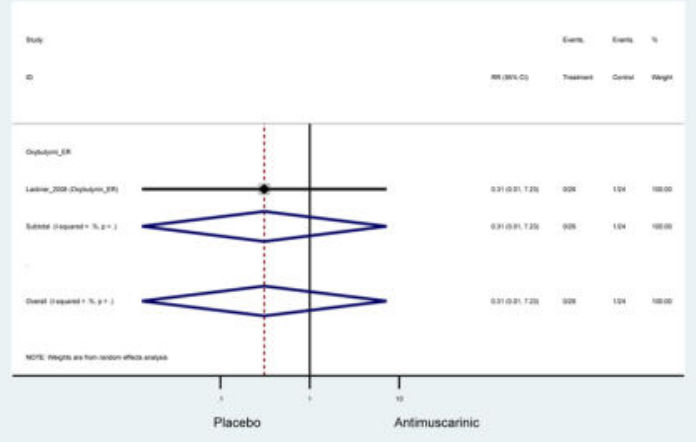

Appendix 9.057: Pruritus (Antimuscarinics versus Placebo)

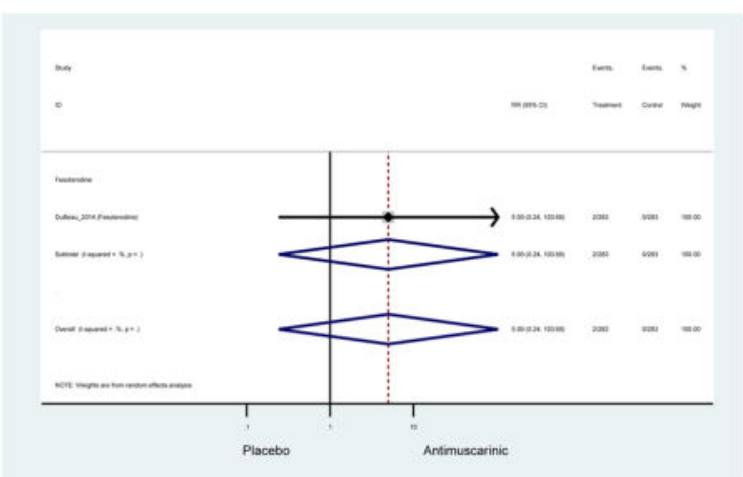

Appendix 9.058: Rash (Antimuscarinics versus Placebo)

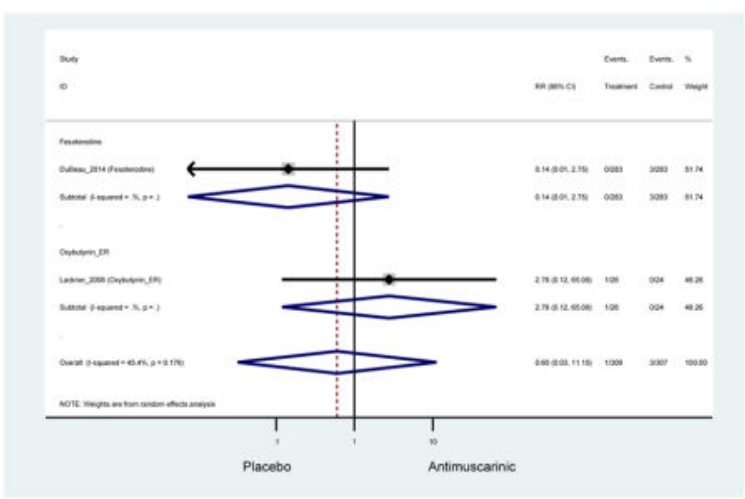

Appendix 9.059: Renal Colic (Antimuscarinics versus Placebo) 


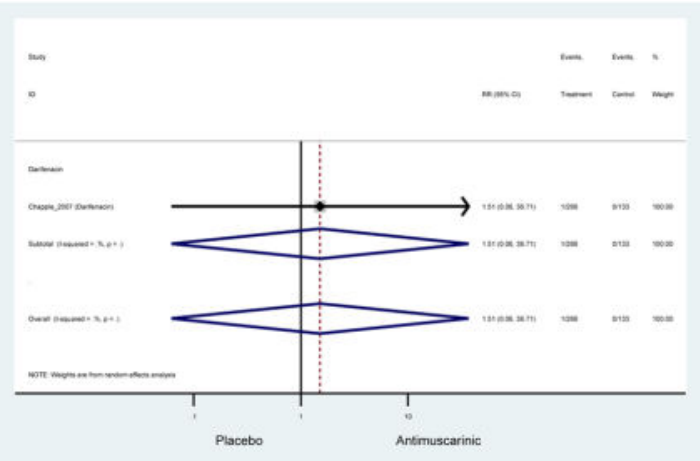

Appendix 9.060: Serious Adverse Events (Antimuscarinics versus Placebo)

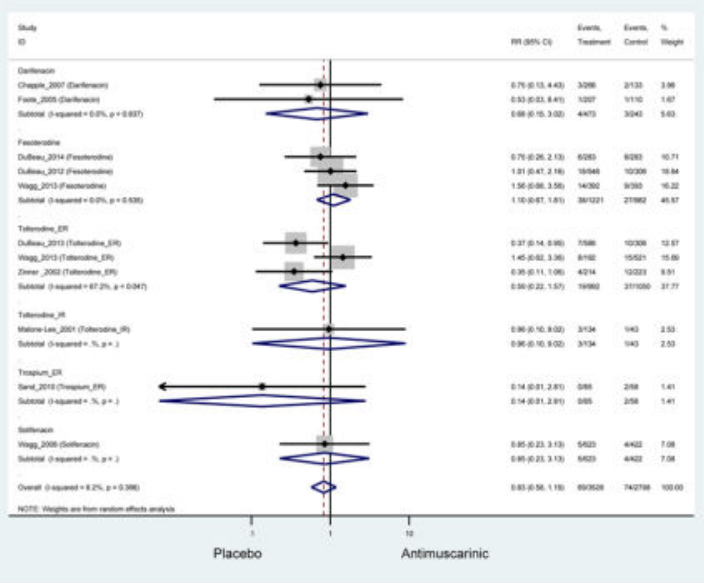

Appendix 9.061: Sinusitis (Antimuscarinics versus Placebo)

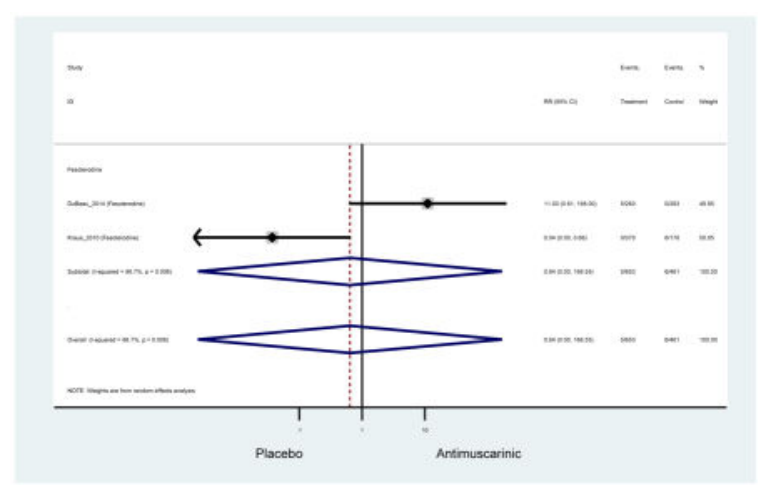

Appendix 9.062: Somnolence (Antimuscarinics versus Placebo)

Arch Gerontol Geriatr. Author manuscript; available in PMC 2018 March 01. 


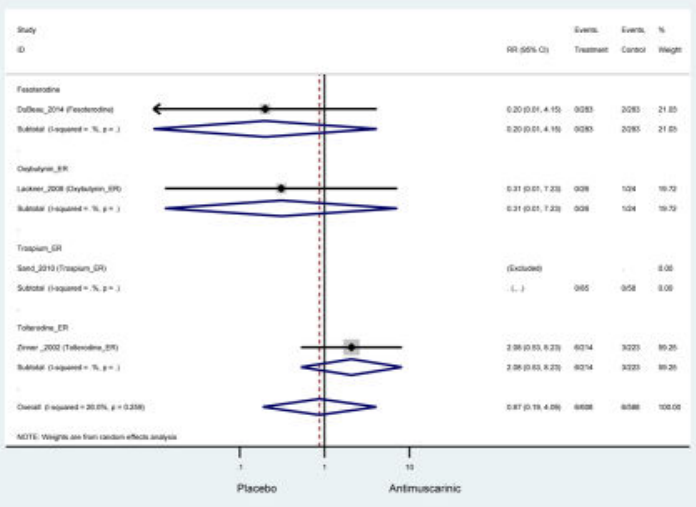

Appendix 9.063: Toothache (Antimuscarinics versus Placebo)

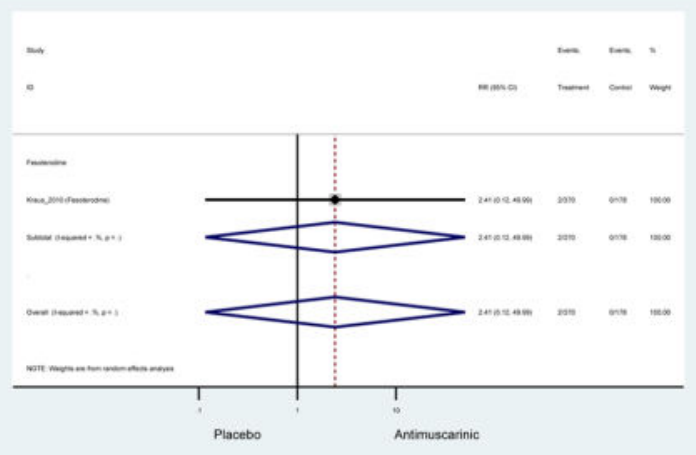

Appendix 9.064: Treatment-related Adverse Events (Antimuscarinics versus Placebo)

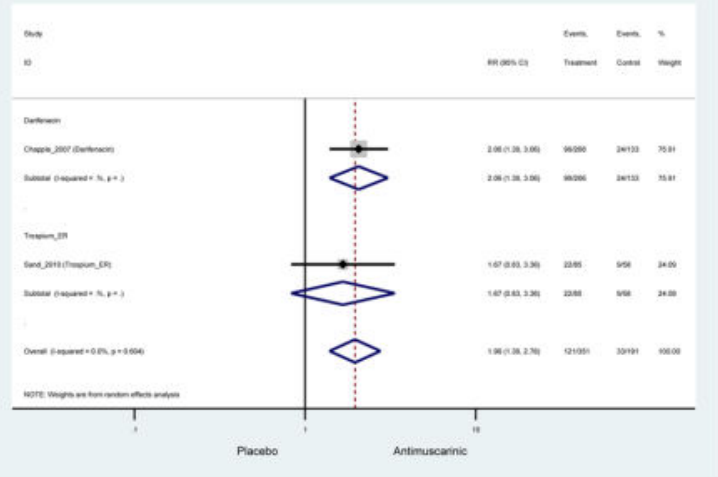

Appendix 9.065: Treatment-emergent Glaucoma (Antimuscarinics versus Placebo)

Zero Outcomes

Appendix 9.066: Upper Respiratory Tract Infection (Antimuscarinics versus Placebo) 


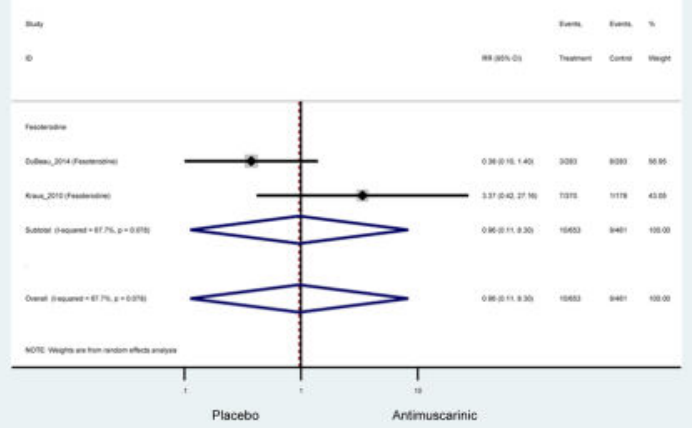

Appendix 9.067: Urinary Retention (Antimuscarinics versus Placebo)

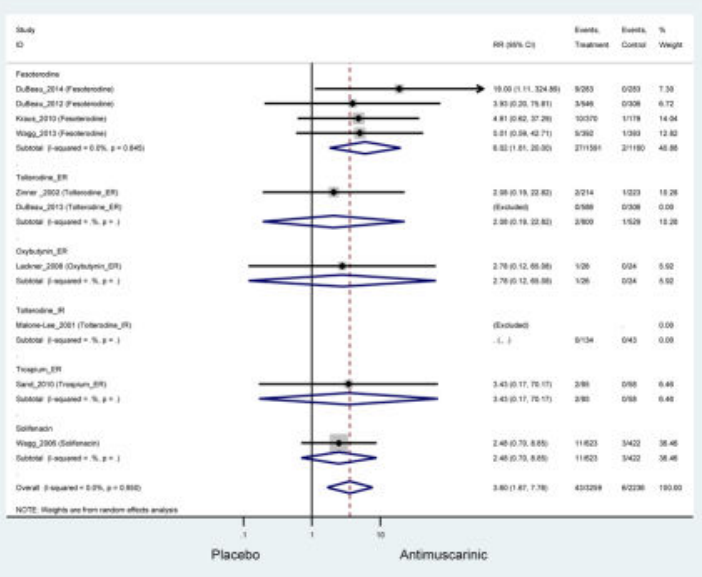

Appendix 9.068: Urinary Tract Infection (Antimuscarinics versus Placebo)

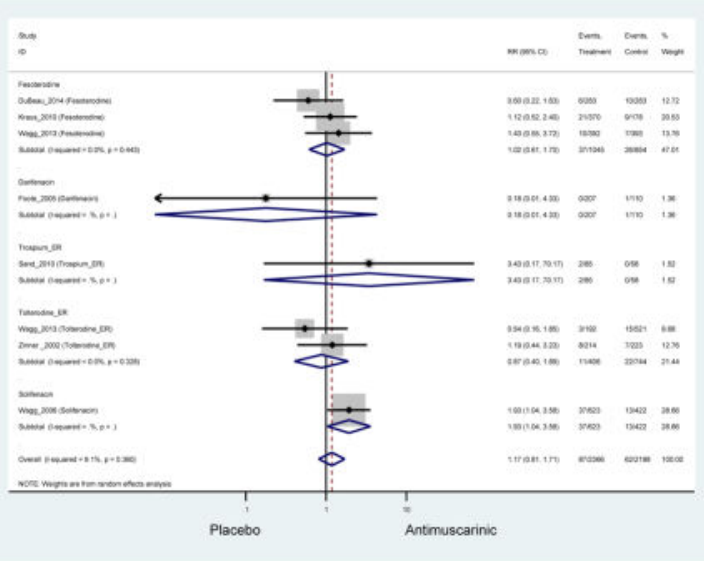

Appendix 9.069: Urinary Tract Infection, Mild-Moderate (Antimuscarinics versus Placebo)

Arch Gerontol Geriatr. Author manuscript; available in PMC 2018 March 01. 


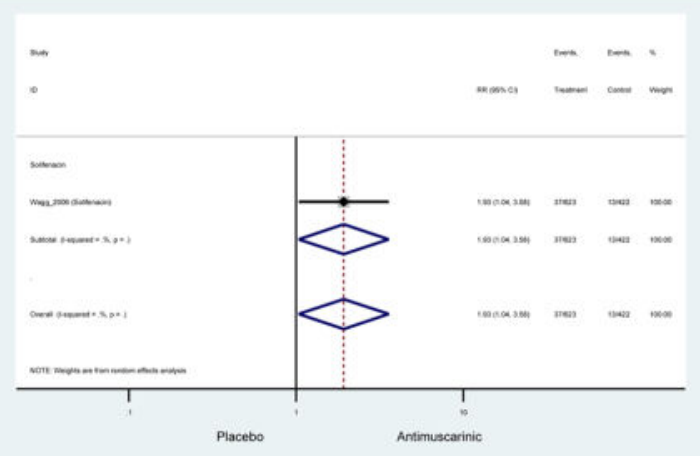

Appendix 9.070: Urinary Tract Infection, Severe (Antimuscarinics versus Placebo)

Zero Outcomes

Appendix 9.071: Urticaria (Antimuscarinics versus Placebo)

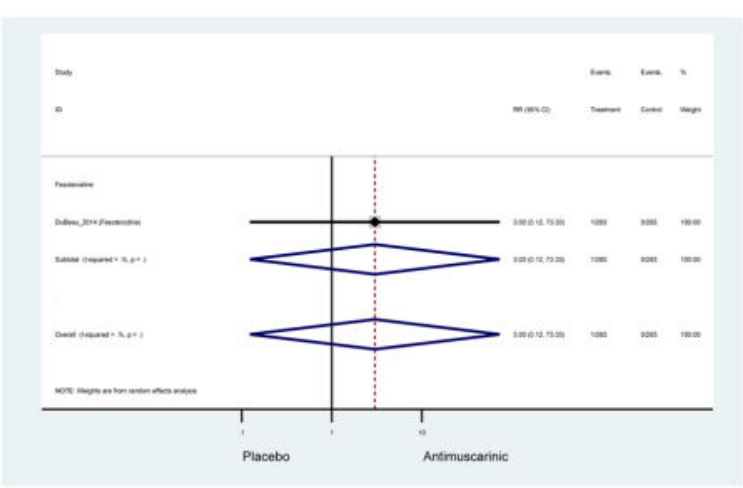

Appendix 9.072: Vertigo (Antimuscarinics versus Placebo)

Zero Outcomes

Appendix 9.073: Visual Disturbance (Antimuscarinics versus Placebo)

Zero Outcomes

Appendix 9.074: Vomiting (Antimuscarinics versus Placebo) 


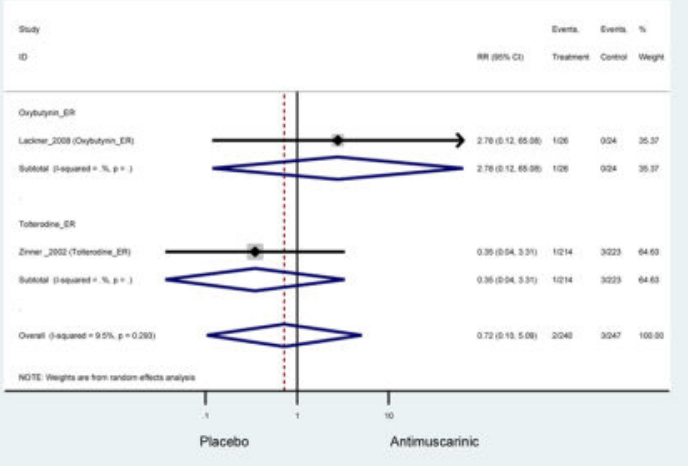

Appendix 9.075: Abdominal Pain (Other Antimuscarinics versus Oxybutynin IR)

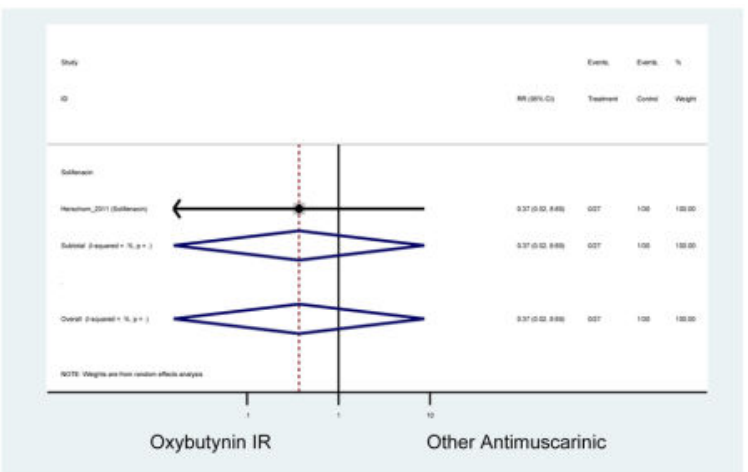

Appendix 9.076: Adverse Events (Other Antimuscarinics versus Oxybutynin IR)

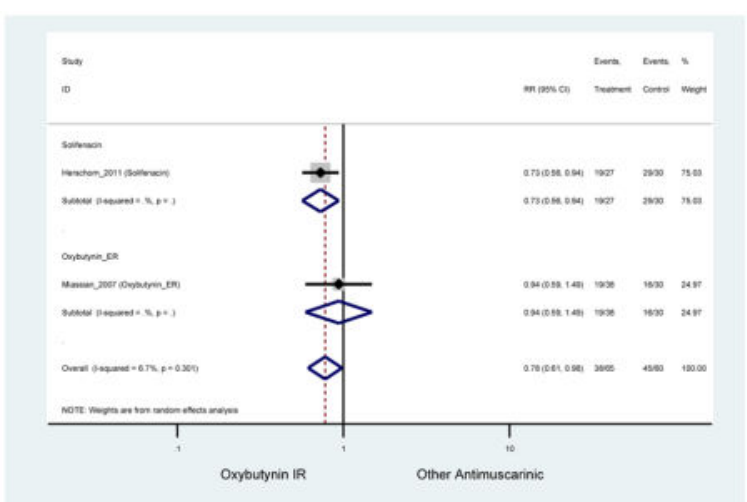

Appendix 9.077: Adverse Events, Mild (Other Antimuscarinics versus Oxybutynin IR) 


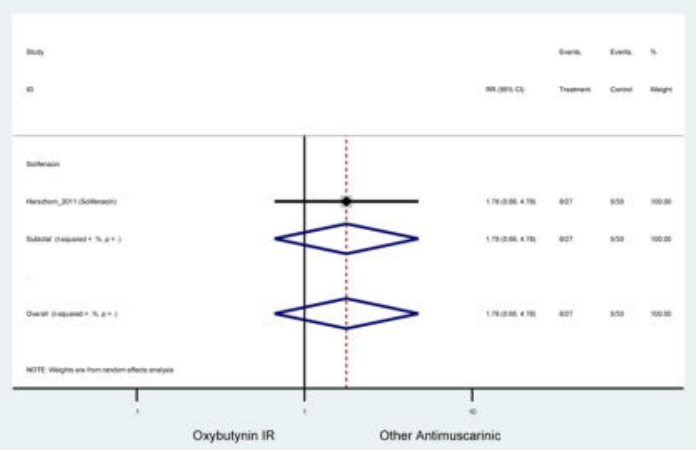

Appendix 9.078: Adverse Events, Moderate (Other Antimuscarinics versus Oxybutynin IR)

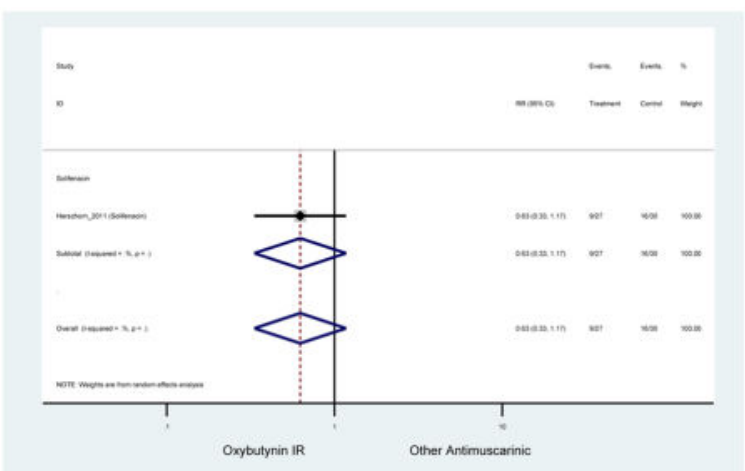

Appendix 9.079: Adverse Events, Severe (Other Antimuscarinics versus Oxybutynin IR)

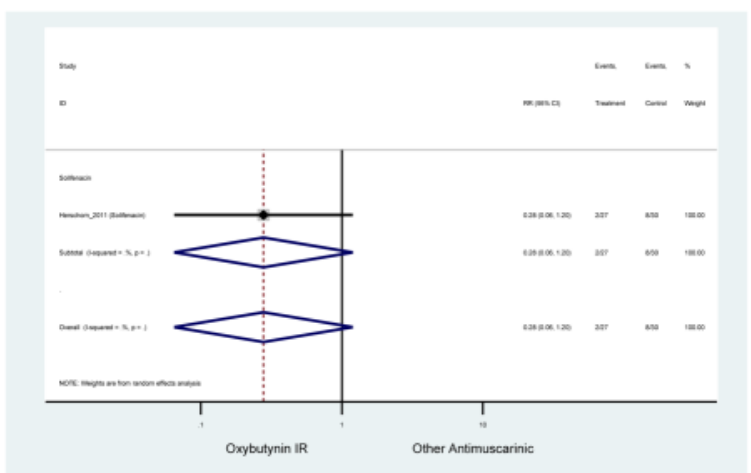

Appendix 9.080: Back Pain (Other Antimuscarinics versus Oxybutynin IR) Zero Outcomes

Appendix 9.081: Confusional State (Other Antimuscarinics versus Oxybutynin IR) 


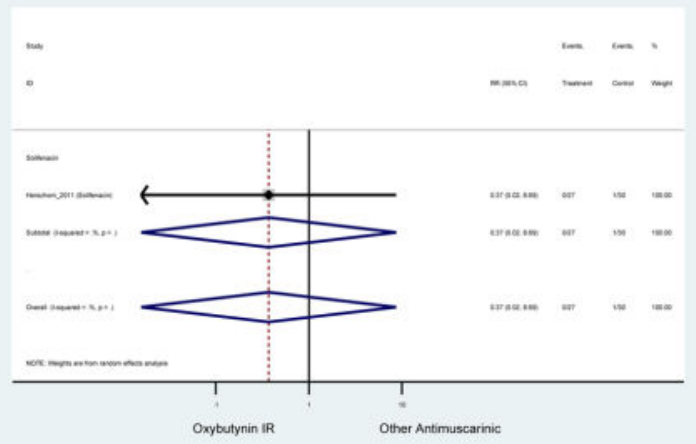

Appendix 9.082: Constipation (Other Antimuscarinics versus Oxybutynin IR)
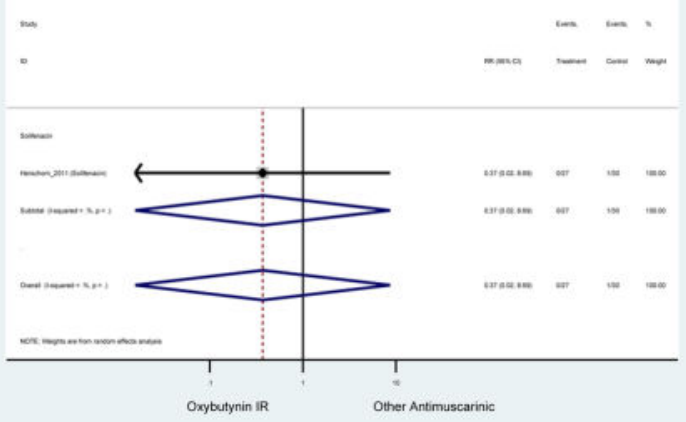

Appendix 9.083: Cough (Other Antimuscarinics versus Oxybutynin IR)

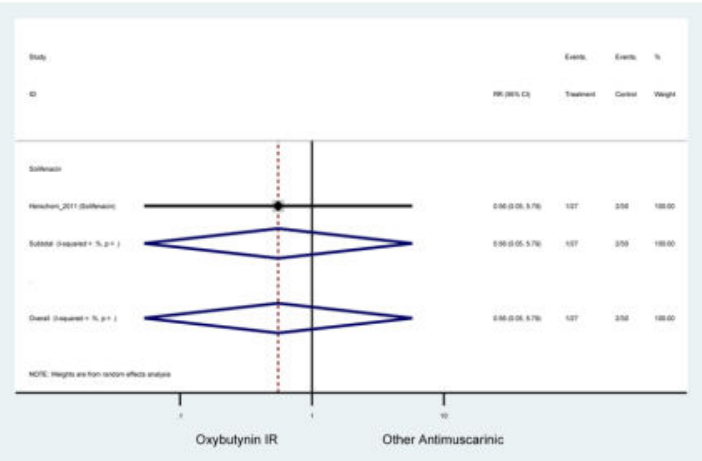

Appendix 9.084: Dizziness (Other Antimuscarinics versus Oxybutynin IR) 


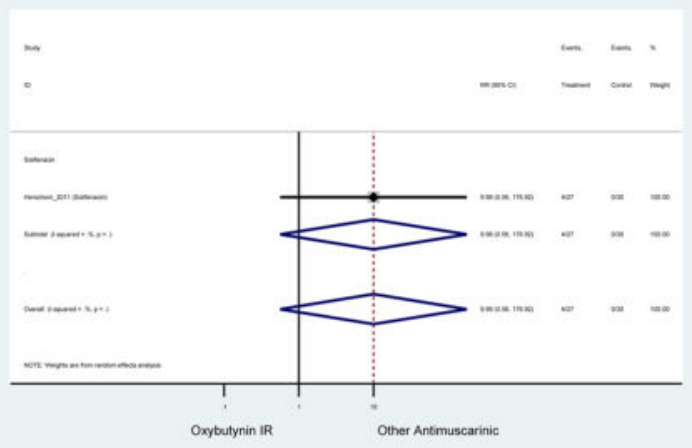

Appendix 9.085: Dry Eyes (Other Antimuscarinics versus Oxybutynin IR)
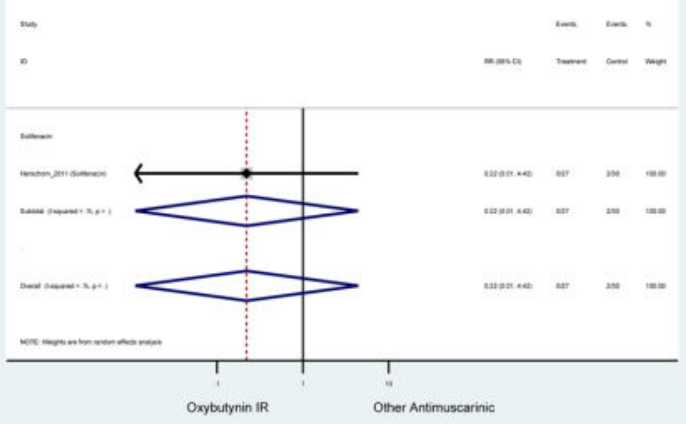

Appendix 9.086: Dry Mouth (Other Antimuscarinics versus Oxybutynin IR)

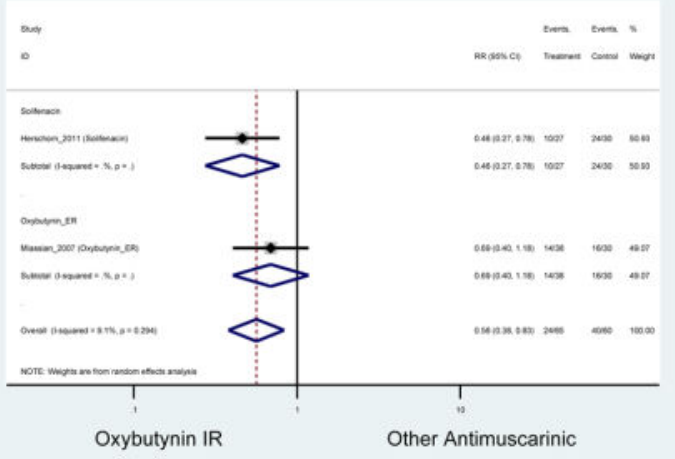

Appendix 9.087: Dry Mouth, Mild (Other Antimuscarinics versus Oxybutynin IR)

Arch Gerontol Geriatr. Author manuscript; available in PMC 2018 March 01. 


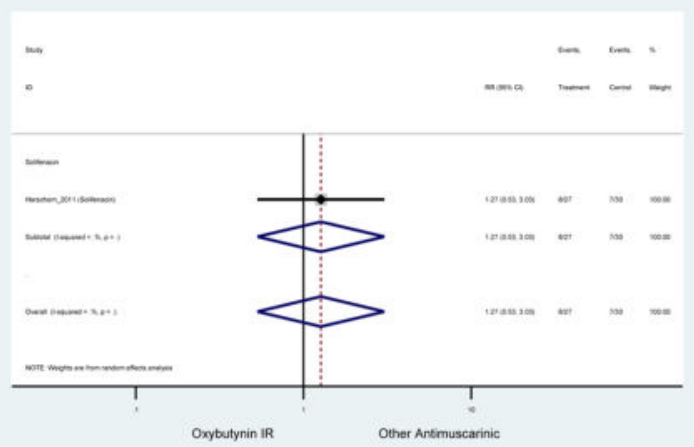

Appendix 9.088: Dry Mouth, Moderate (Other Antimuscarinics versus Oxybutynin IR)

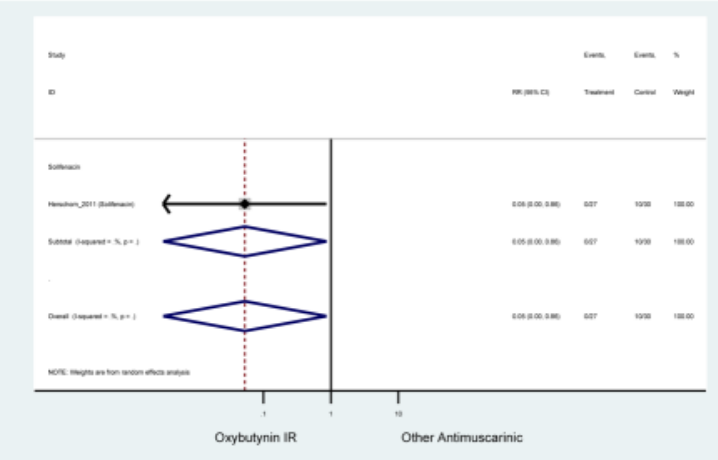

Appendix 9.089: Dry Mouth, Severe (Other Antimuscarinics versus Oxybutynin IR)
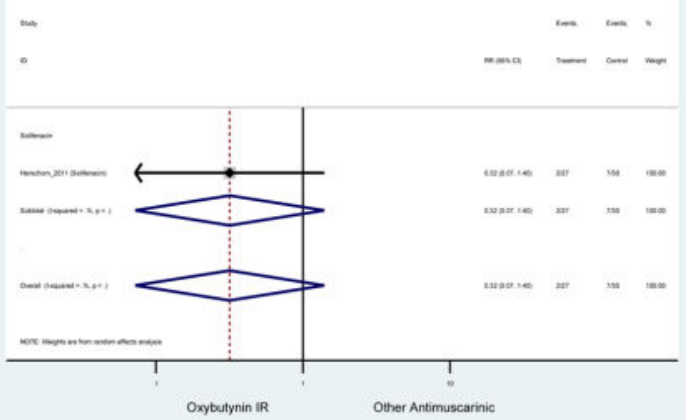

Appendix 9.090: Dry Skin (Other Antimuscarinics versus Oxybutynin IR) Zero Outcomes

Appendix 9.091: Dry Throat (Other Antimuscarinics versus Oxybutynin IR)

Zero outcomes

Appendix 9.092: Dysgeusia (Other Antimuscarinics versus Oxybutynin IR) 


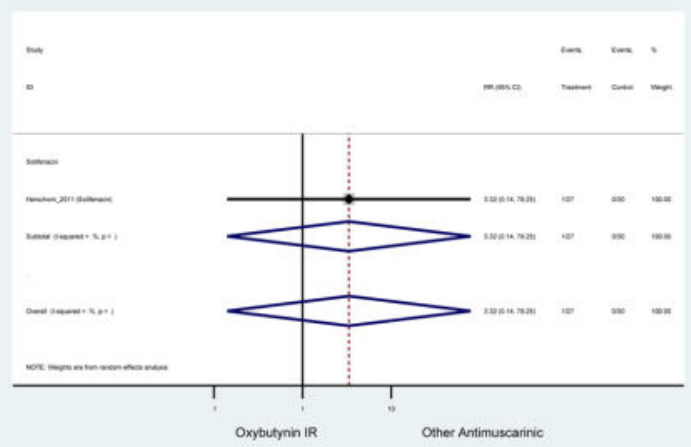

Appendix 9.093: Dyspepsia (Other Antimuscarinics versus Oxybutynin IR)
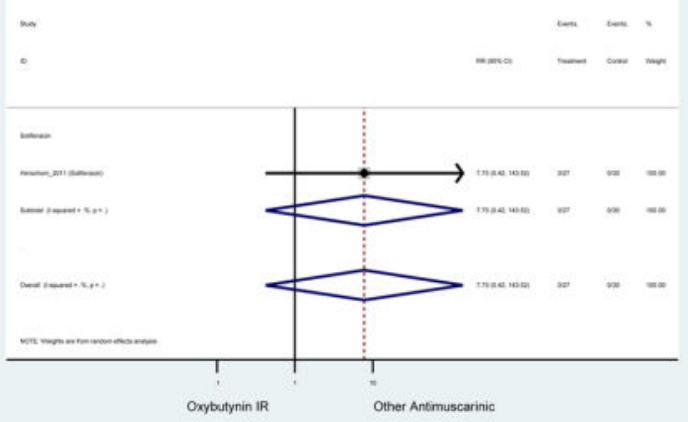

Appendix 9.094: Dysphagia (Other Antimuscarinics versus Oxybutynin IR)

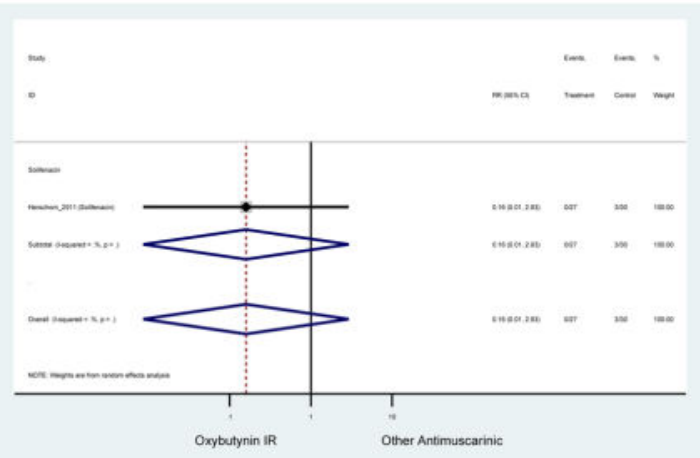

Appendix 9.095: Dysphonia (Other Antimuscarinics versus Oxybutynin IR) 


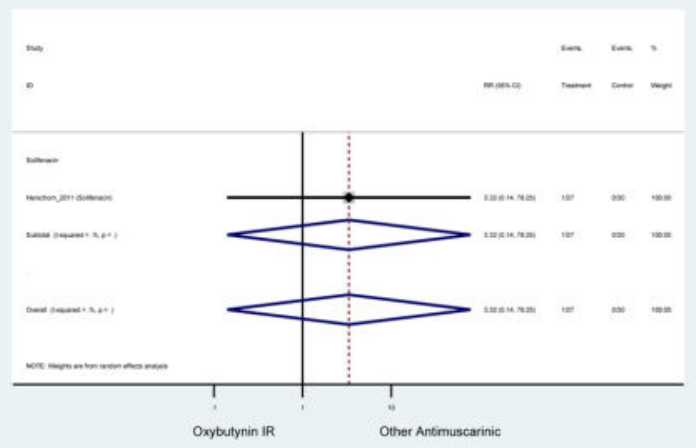

Appendix 9.096: Epistaxis (Other Antimuscarinics versus Oxybutynin IR)

Zero Outcomes

Appendix 9.097: Fatigue (Other Antimuscarinics versus Oxybutynin IR)

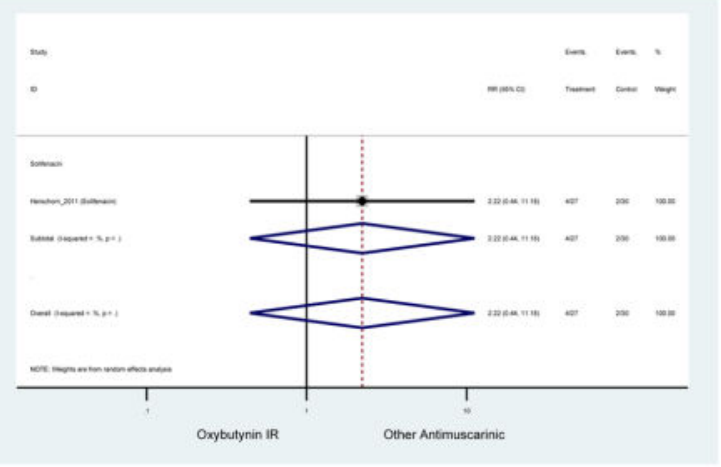

Appendix 9.098: Gastrointestinal (Other Antimuscarinics versus Oxybutynin IR)

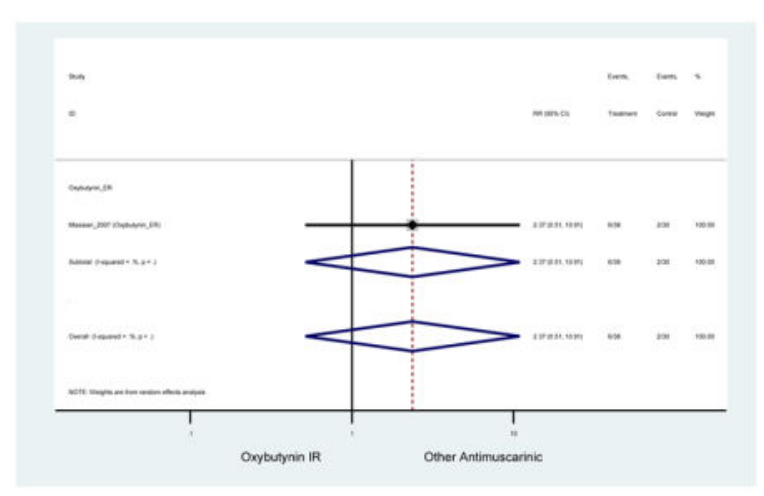

Appendix 9.099: Headache (Other Antimuscarinics versus Oxybutynin IR)

Arch Gerontol Geriatr. Author manuscript; available in PMC 2018 March 01. 


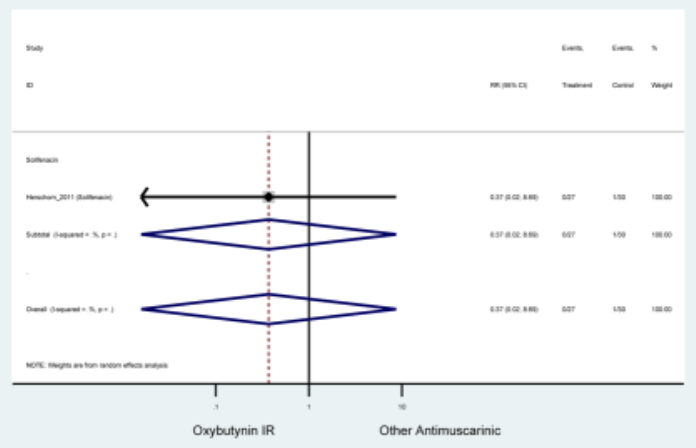

Appendix 9.100: Nasopharyngitis (Other Antimuscarinics versus Oxybutynin IR)
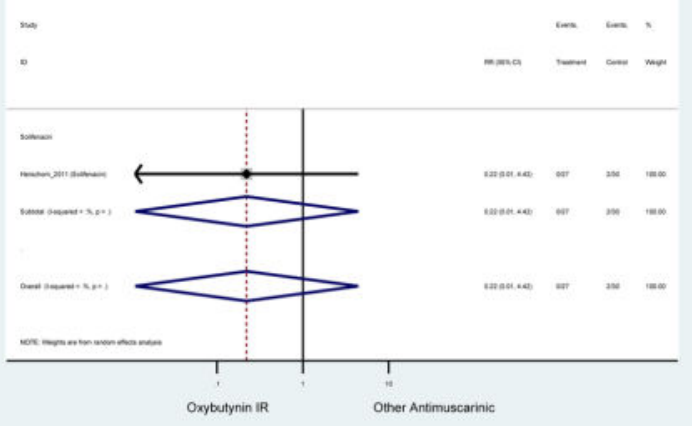

Appendix 9.101: Nausea (Other Antimuscarinics versus Oxybutynin IR)

Zero Outcomes

Appendix 9.102: Somnolence (Other Antimuscarinics versus Oxybutynin IR)

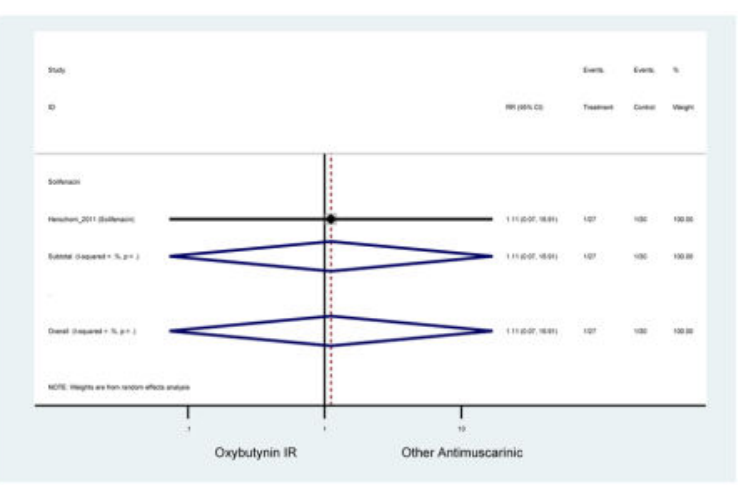

Appendix 9.103: Treatment-related Adverse Events (Other Antimuscarinics versus Oxybutynin IR) 


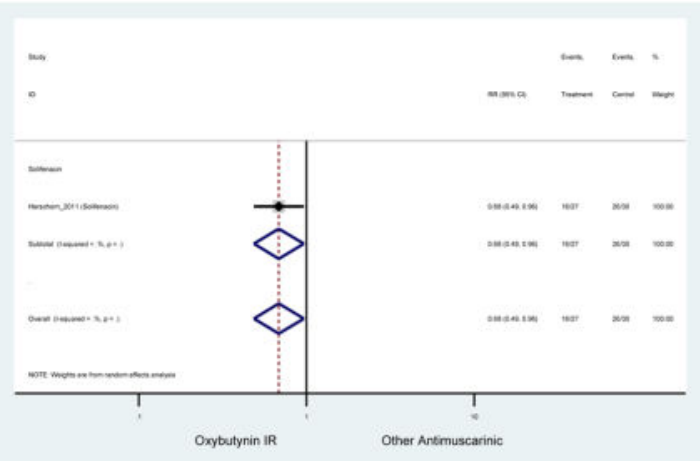

Appendix 9.104: Urinary Tract Infections (Other Antimuscarinics versus Oxybutynin IR)

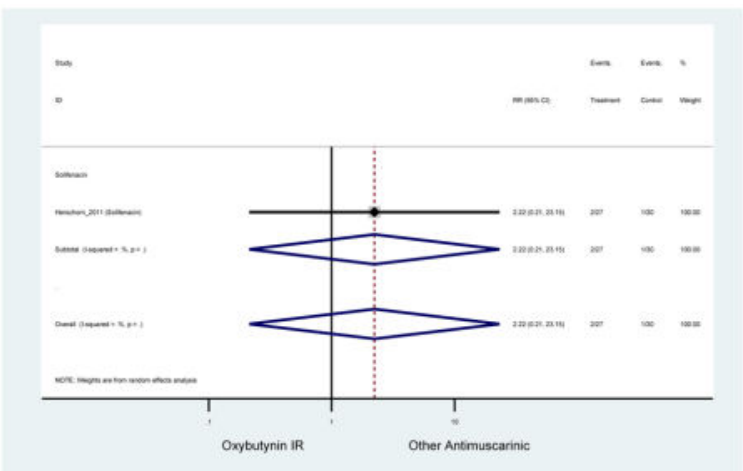

Appendix 9.105: Balance Disorder (Other Antimuscarinics versus Tolterodine IR)

Zero Outcomes

Appendix 9.106: Blurred Vision (Other Antimuscarinics versus Tolterodine IR)

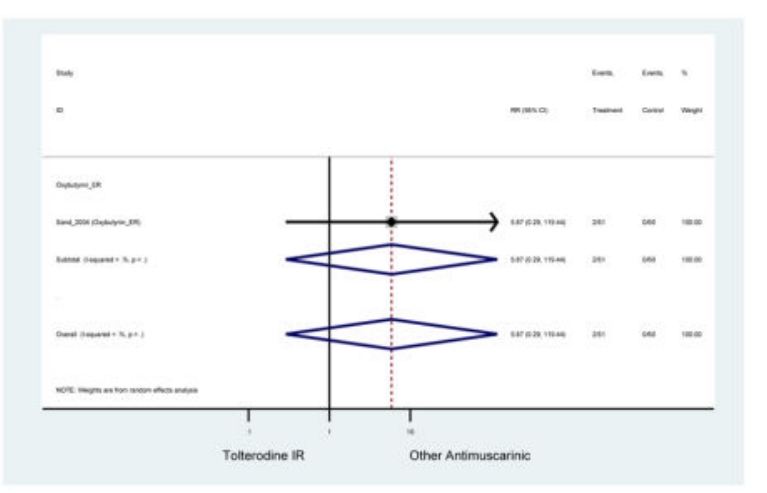

Appendix 9.107: Confusional State (Other Antimuscarinics versus Tolterodine IR)

Zero Outcomes

Appendix 9.108: Constipation (Other Antimuscarinics versus Tolterodine IR) 


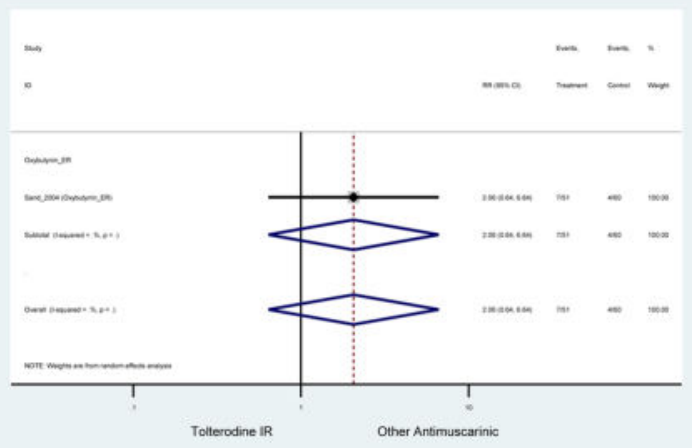

Appendix 9.109: Dizziness (Other Antimuscarinics versus Tolterodine IR)

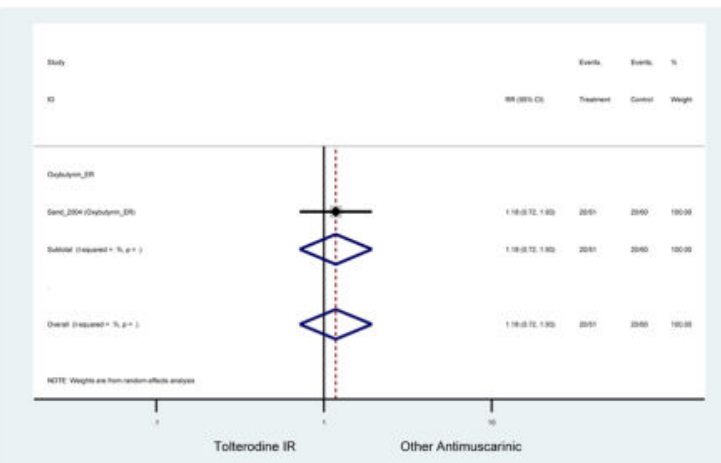

Appendix 9.110: Dry Mouth (Other Antimuscarinics versus Tolterodine IR)

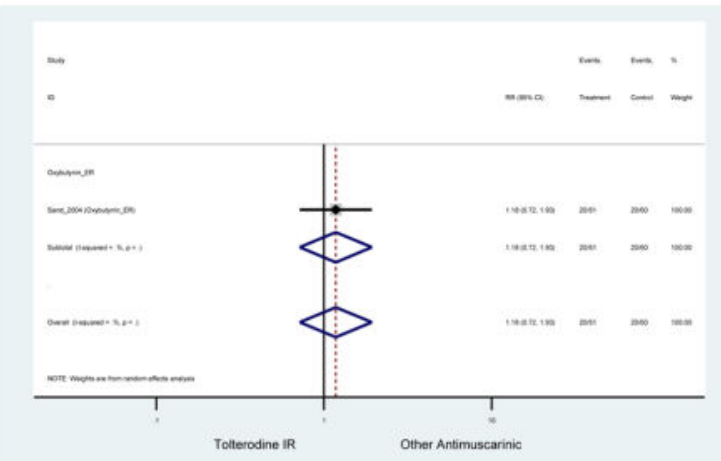

Appendix 9.111: Dyspepsia (Other Antimuscarinics versus Tolterodine IR) 


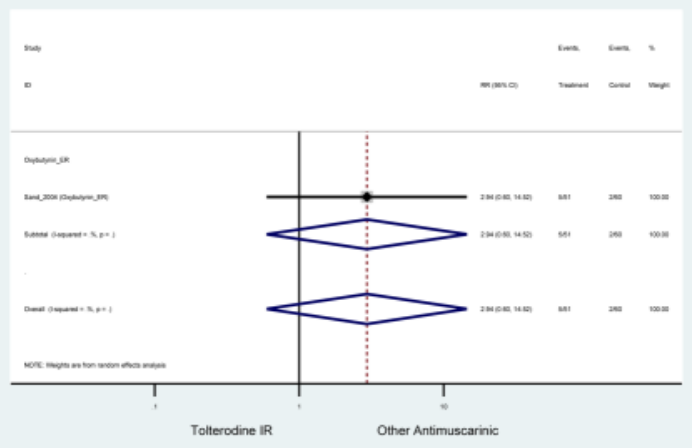

Appendix 9.112: Headache (Other Antimuscarinics versus Tolterodine IR)
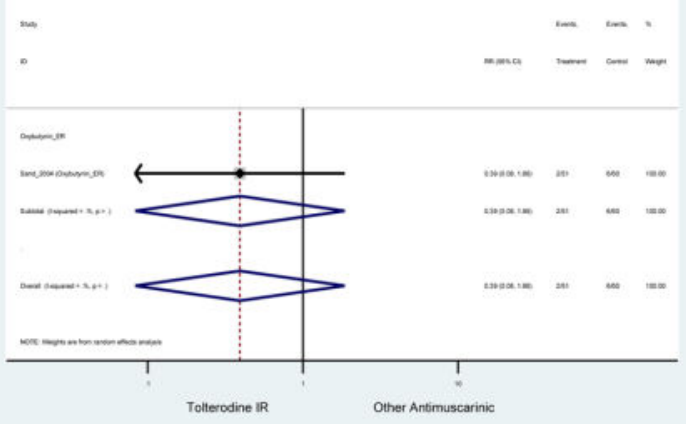

Appendix 9.113: Insomnia (Other Antimuscarinics versus Tolterodine IR)

Zero Outcomes

Appendix 9.114: Nausea (Other Antimuscarinics versus Tolterodine IR)

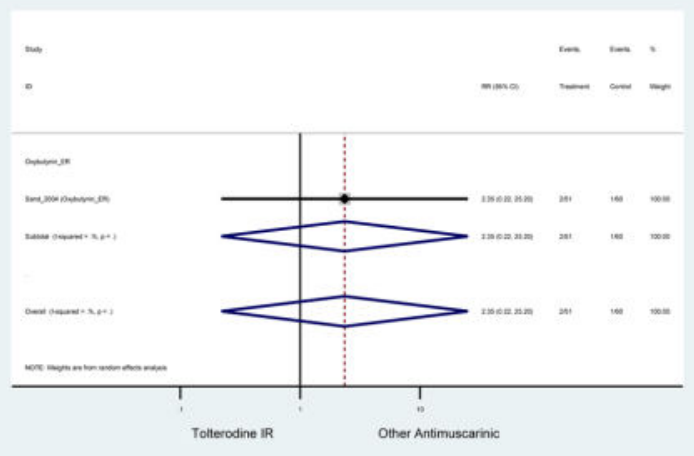

Appendix 9.115: Nervousness (Other Antimuscarinics versus Tolterodine IR) 


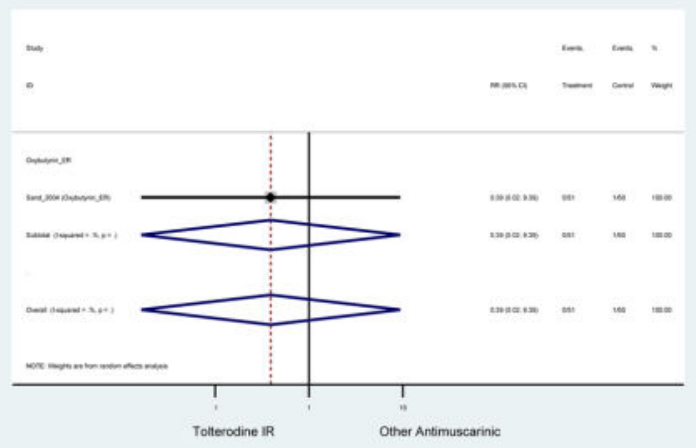

Appendix 9.116: Somnolence (Other Antimuscarinics versus Tolterodine IR)

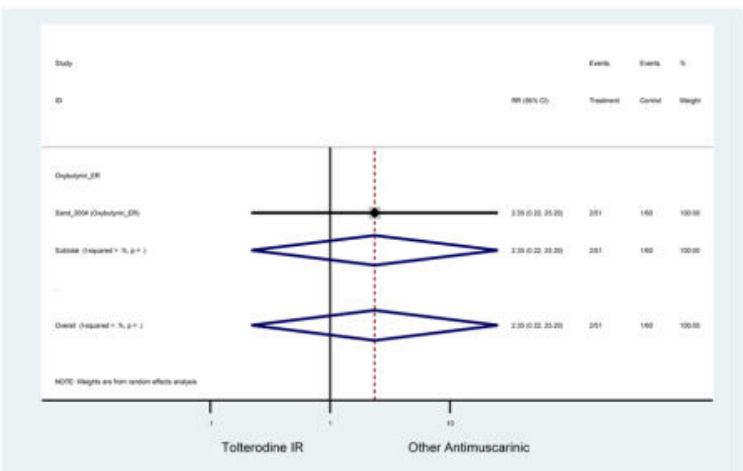

Appendix 9.117: Urinary Retention (Other Antimuscarinics versus Tolterodine IR)

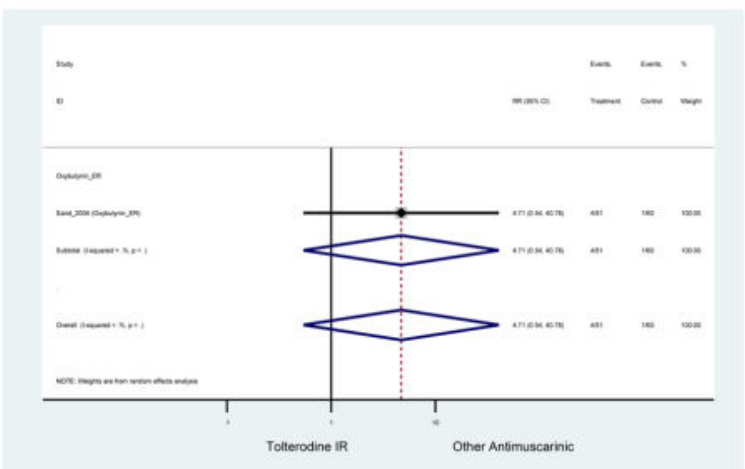

Appendix 9.118: Vomiting (Other Antimuscarinics versus Tolterodine IR) 


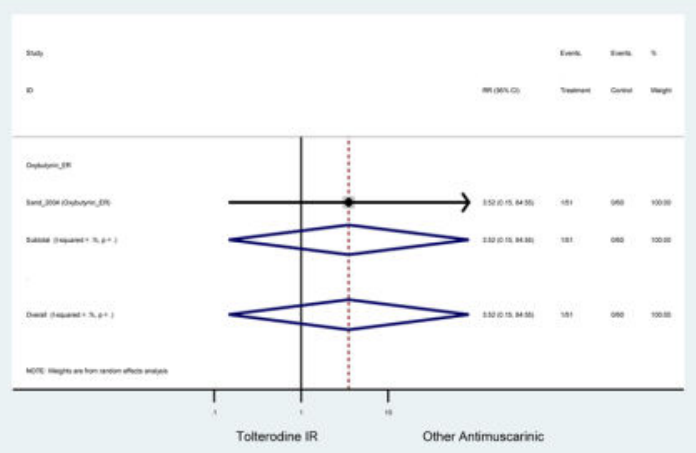

Appendix 9.119: Adverse Events (Other Antimuscarinics versus Tolterodine ER)

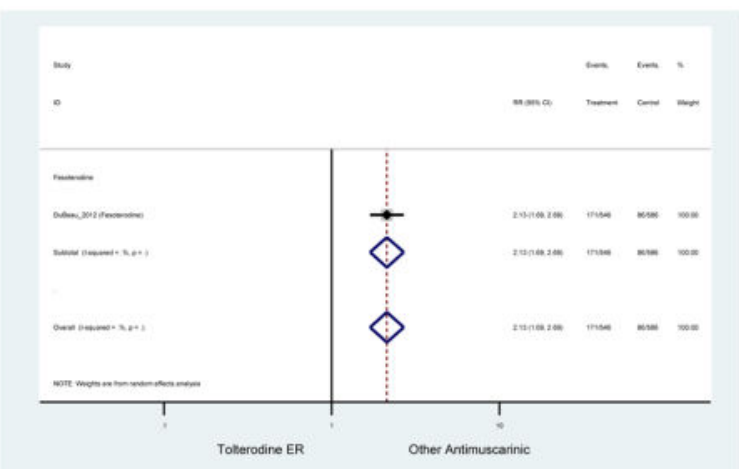

Appendix 9.120: Constipation (Other Antimuscarinics versus Tolterodine ER)

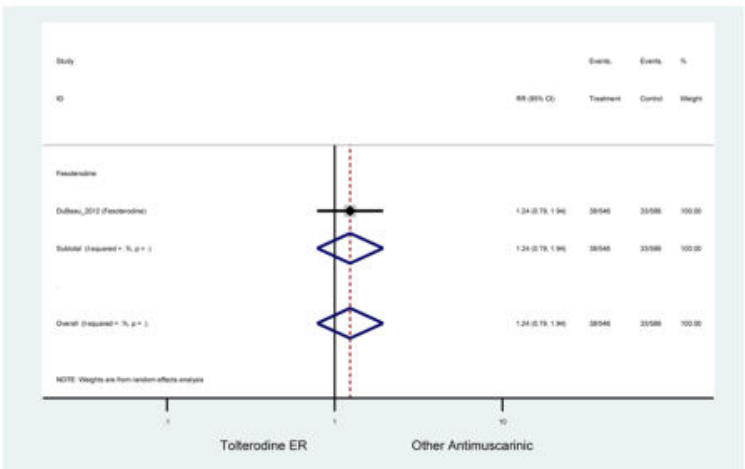

Appendix 9.121: Diarrhea (Other Antimuscarinics versus Tolterodine ER) 


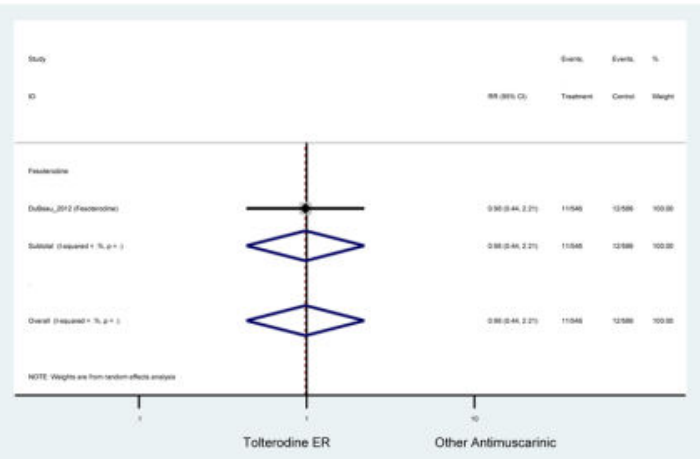

Appendix 9.122: Dry Mouth (Other Antimuscarinics versus Tolterodine ER)

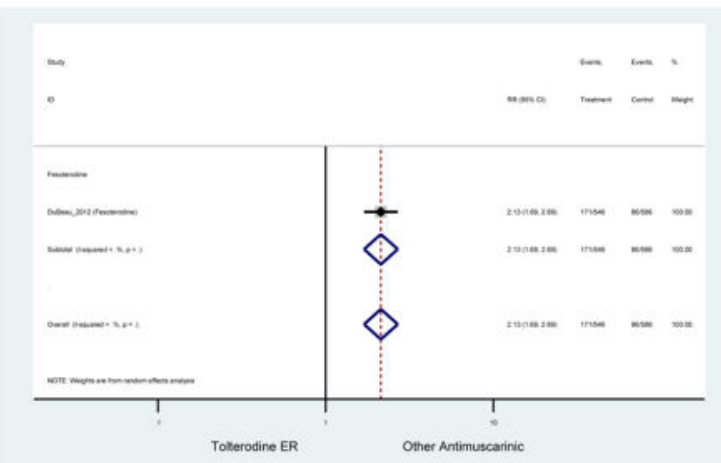

Appendix 9.123: Headache (Other Antimuscarinics versus Tolterodine ER)

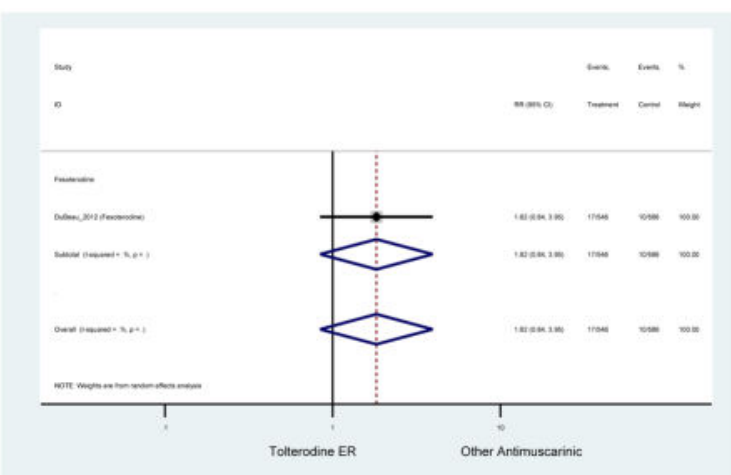

Appendix 9.124: Influenza (Other Antimuscarinics versus Tolterodine ER) 


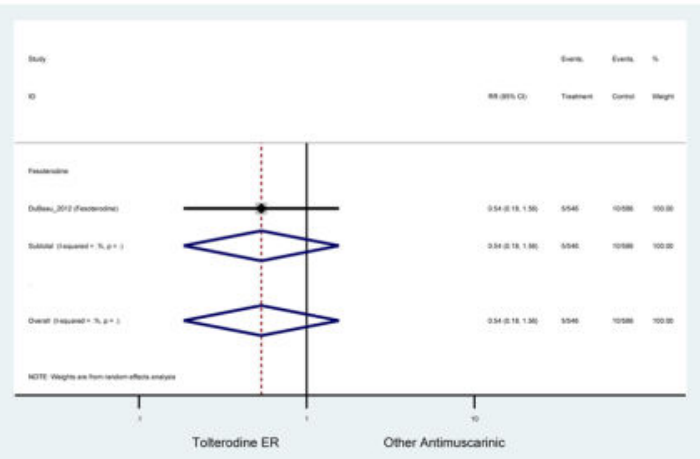

Appendix 9.125: Serious Adverse Events (Other Antimuscarinics versus Tolterodine ER)

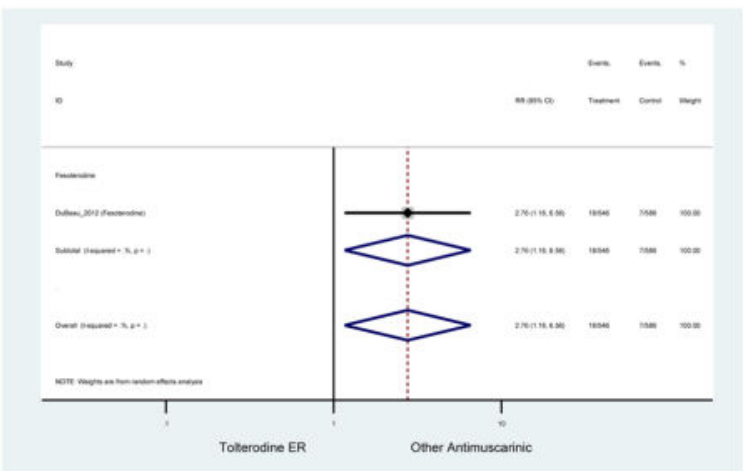

Appendix 9.126: Urinary Retention (Other Antimuscarinics versus Tolterodine ER)

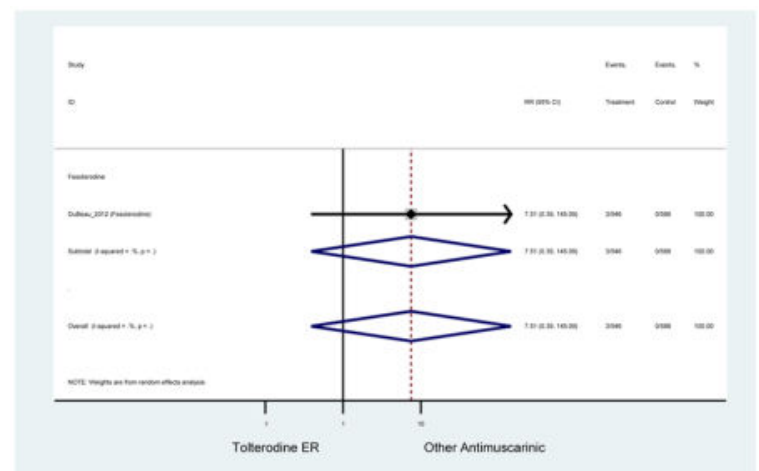

Appendix 9.127: Administrative Problems - Treatment Discontinuation (Antimuscarinics versus Placebo) 


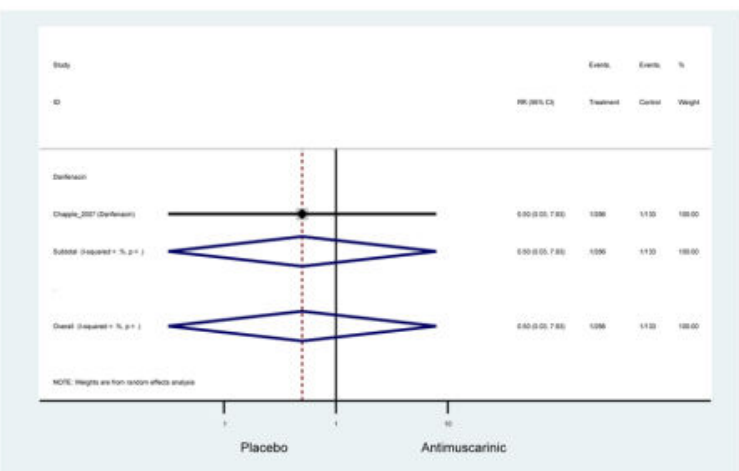

Appendix 9.128: Adverse Events - Treatment Discontinuation (Antimuscarinics versus Placebo)

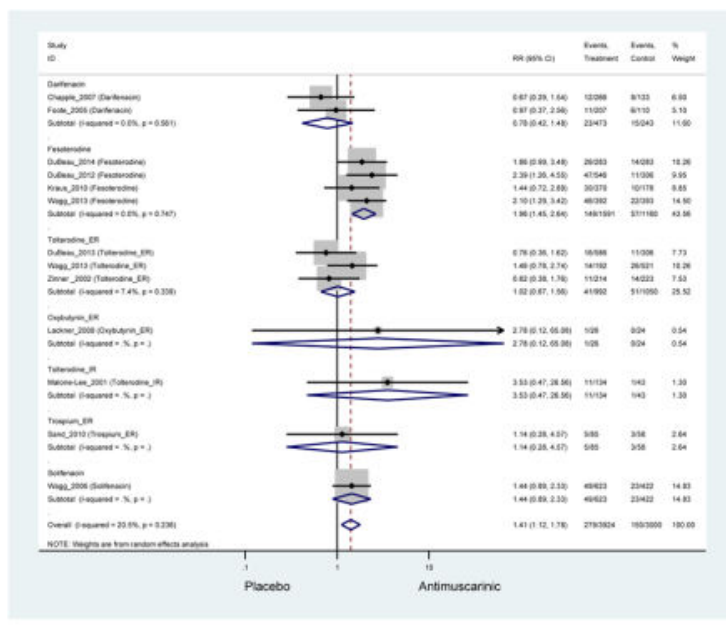

Appendix 9.129: Cognitive Function Adverse Events - Treatment Discontinuation (Antimuscarinics versus Placebo)

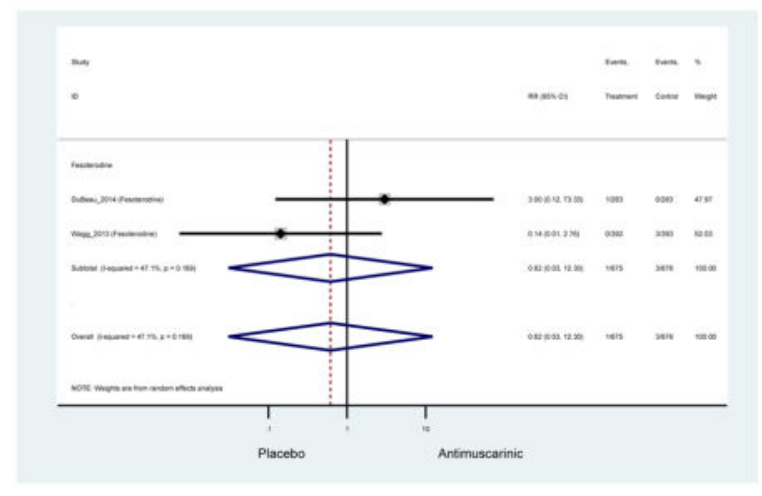

Appendix 9.130: Constipation - Treatment Discontinuation (Antimuscarinics versus Placebo) 


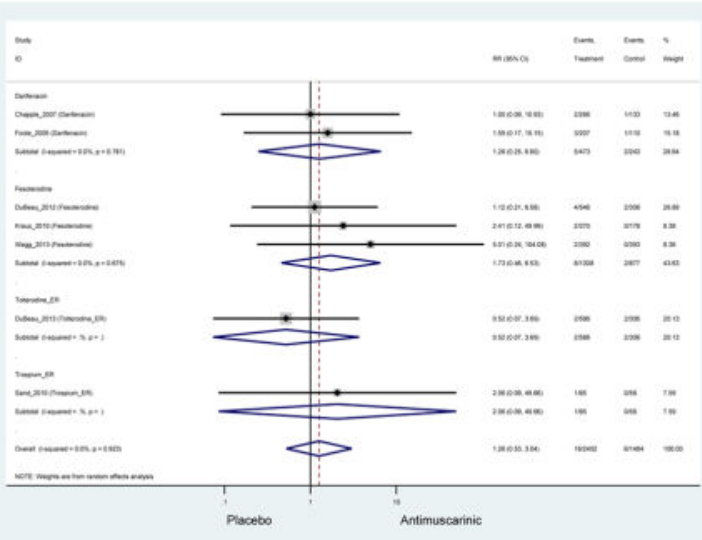

Appendix 9.131: Death - Treatment Discontinuation (Antimuscarinics versus Placebo)

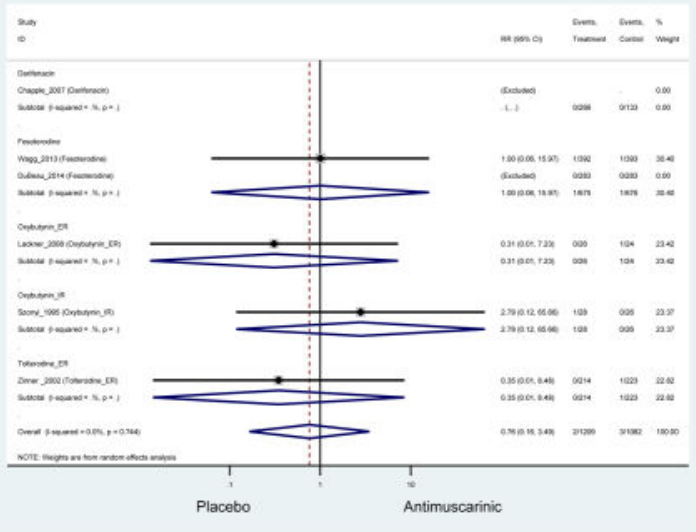

Appendix 9.132: Decline in Medical Condition - Treatment Discontinuation (Antimuscarinics versus Placebo)

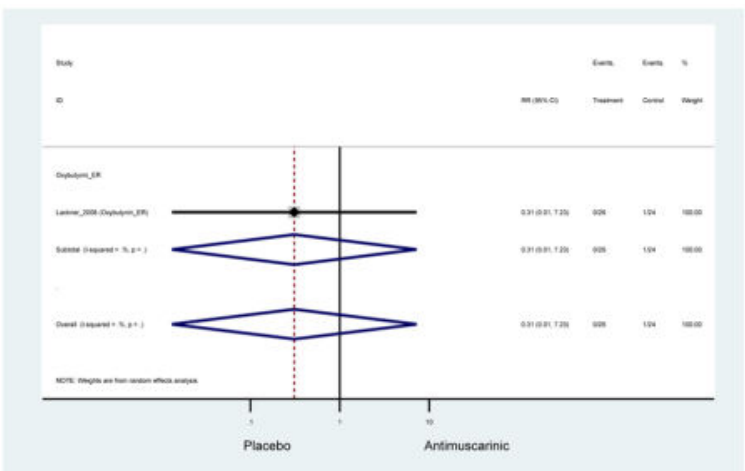

Appendix 9.133: Did Not Meet Entrance Criteria - Treatment Discontinuation (Antimuscarinics versus Placebo) 


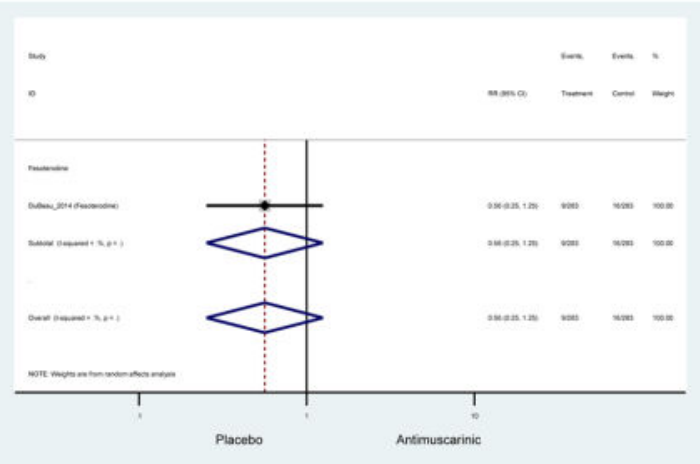

Appendix 9.134: Dizziness - Treatment Discontinuation (Antimuscarinics versus Placebo)

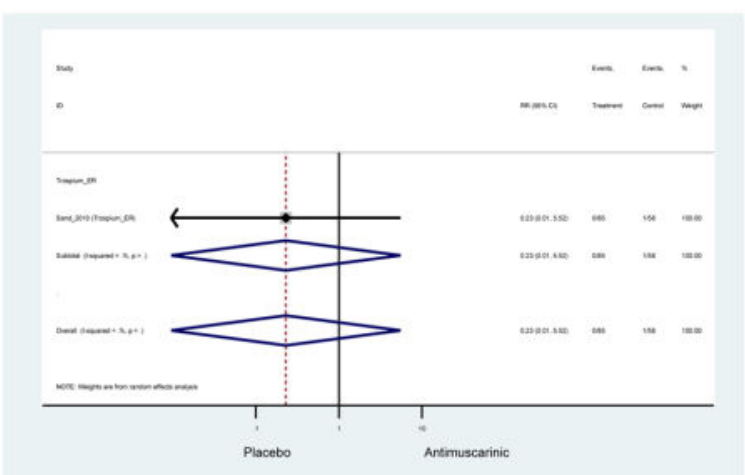

Appendix 9.135: Dry Mouth - Treatment Discontinuation (Antimuscarinics versus Placebo)

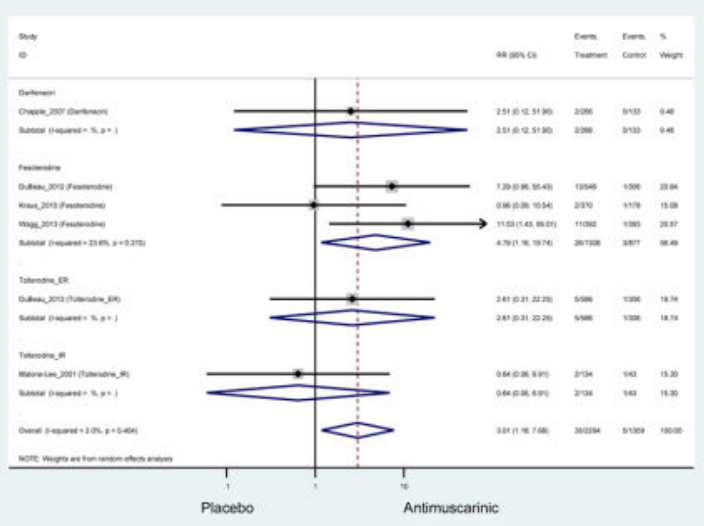

Appendix 9.136: Dry Throat - Treatment Discontinuation (Antimuscarinics versus Placebo) 


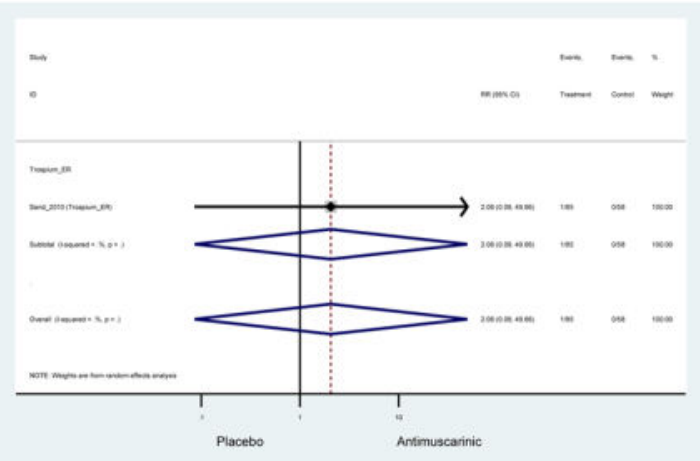

Appendix 9.137: Fall - Treatment Discontinuation (Antimuscarinics versus Placebo)
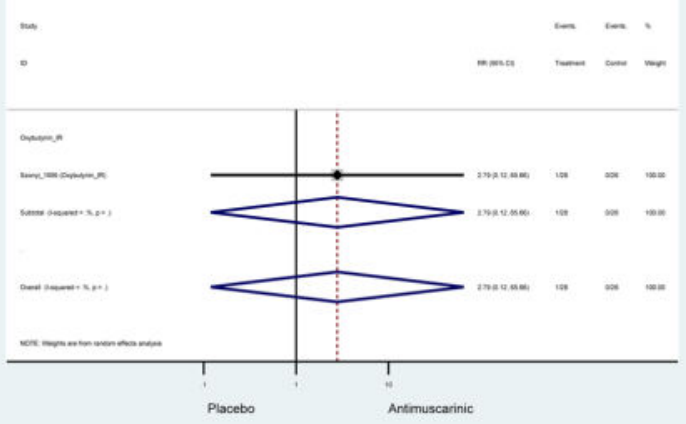

Appendix 9.138: Flatulence - Treatment Discontinuation (Antimuscarinics versus Placebo)

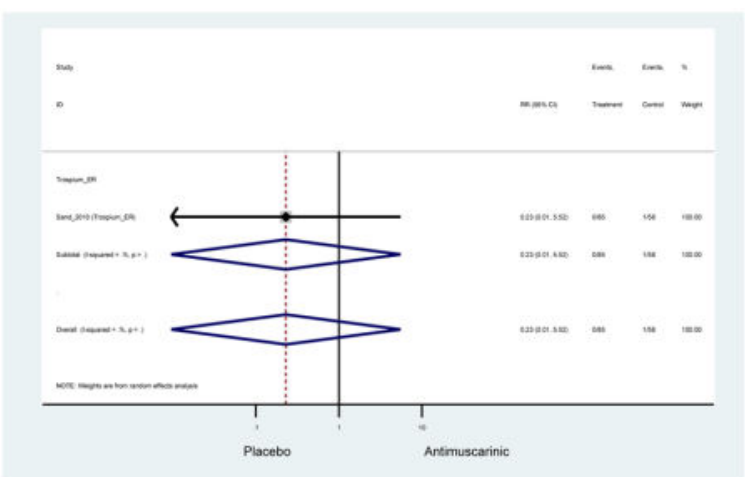

Appendix 9.139: Headache - Treatment Discontinuation (Antimuscarinics versus Placebo) 


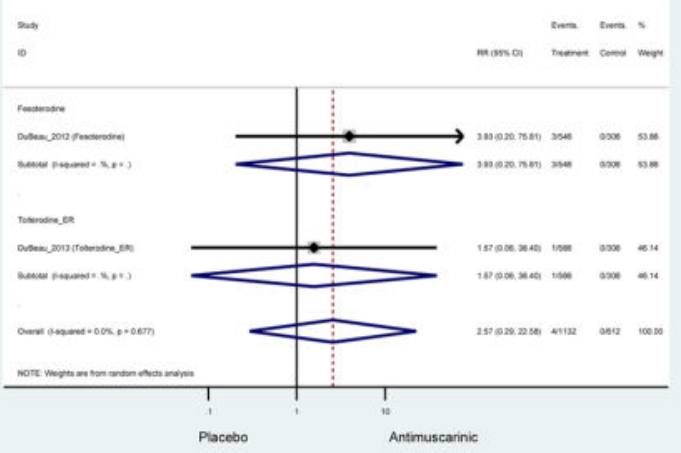

Appendix 9.140: Heartburn/Gastroesophageal Reflux Disorder - Treatment Discontinuation (Antimuscarinics versus Placebo)

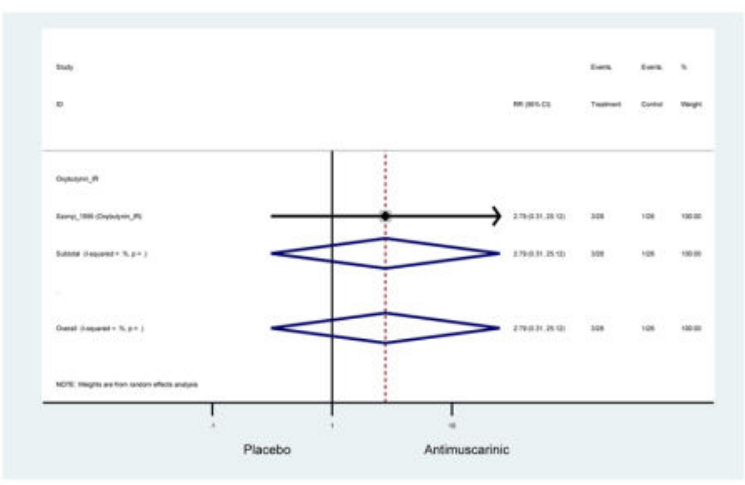

Appendix 9.141: Lost to Follow-up - Treatment Discontinuation (Antimuscarinics versus Placebo)

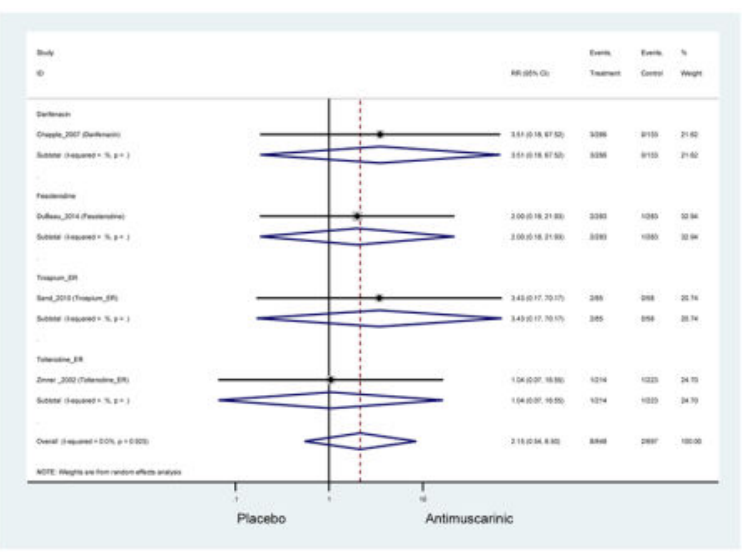

Appendix 9.142: Other - Treatment Discontinuation (Antimuscarinics versus Placebo)

Arch Gerontol Geriatr. Author manuscript; available in PMC 2018 March 01. 


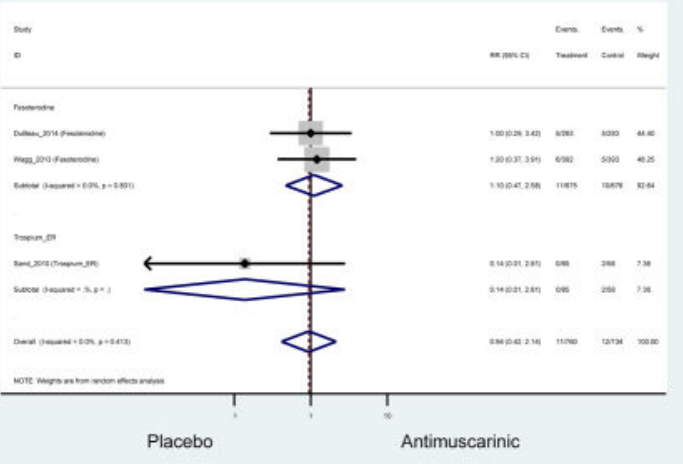

Appendix 9.143: Protocol Violation - Treatment Discontinuation (Antimuscarinics versus Placebo)

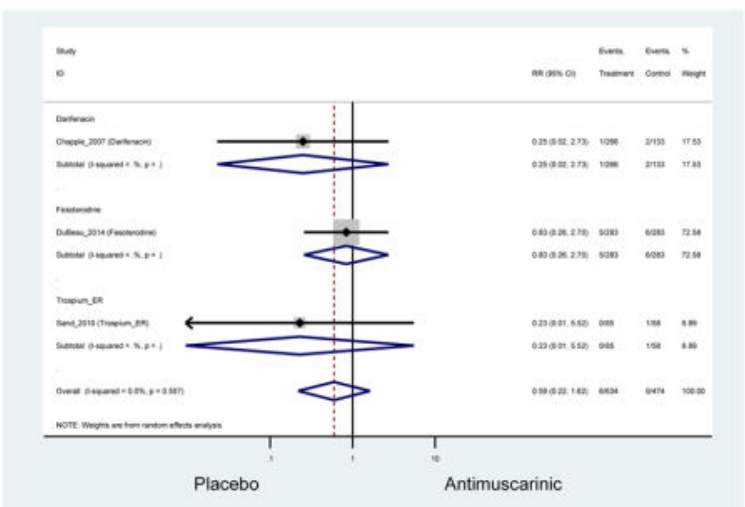

Appendix 9.144: Renal Pain - Treatment Discontinuation (Antimuscarinics versus Placebo)

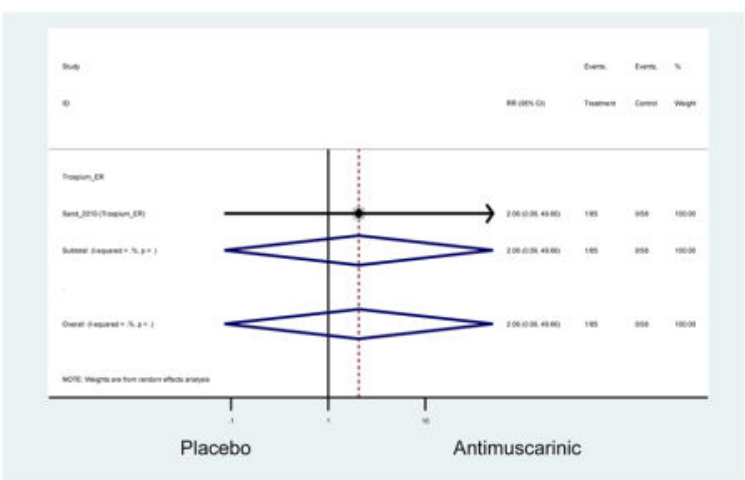

Appendix 9.145: Serious Adverse Events - Treatment Discontinuation (Antimuscarinics versus Placebo) 


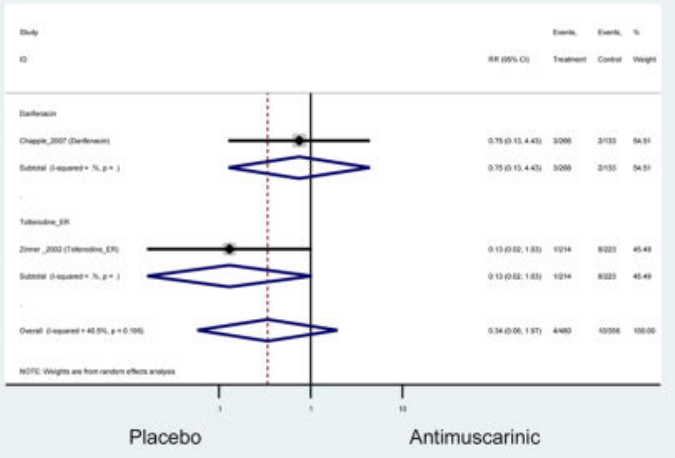

Appendix 9.146: Subject Non-Compliance - Treatment Discontinuation (Antimuscarinics versus Placebo)

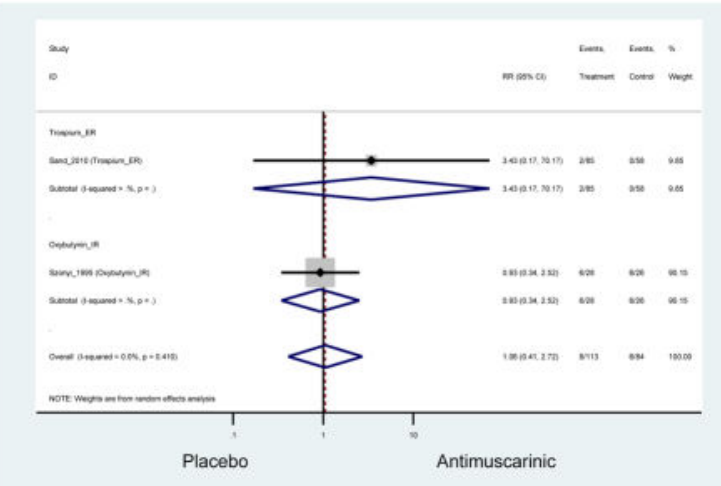

Appendix 9.147: Total Discontinuation - Treatment Discontinuation (Antimuscarinics versus Placebo)

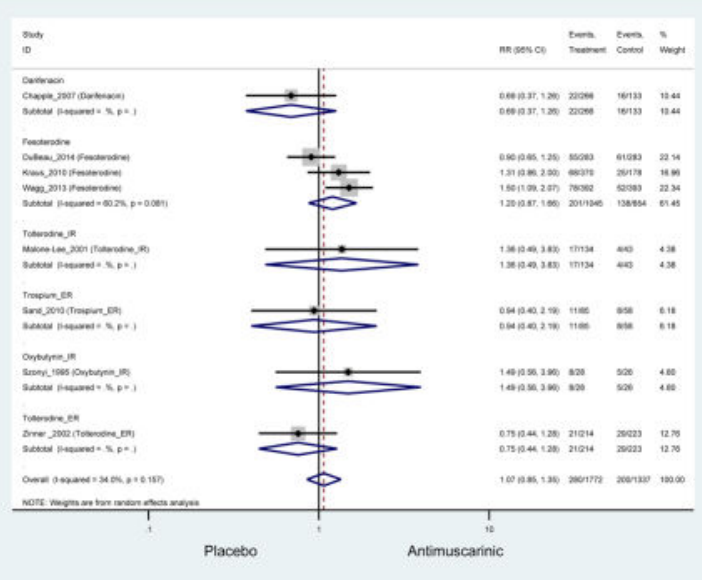

Appendix 9.148: Unsatisfactory Therapeutic Effect - Treatment Discontinuation (Antimuscarinics versus Placebo) 


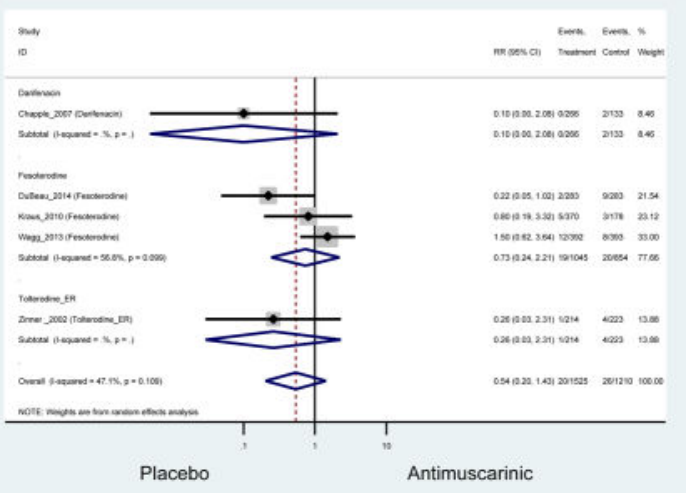

Appendix 9.149: Urinary Retention - Treatment Discontinuation (Antimuscarinics versus Placebo)

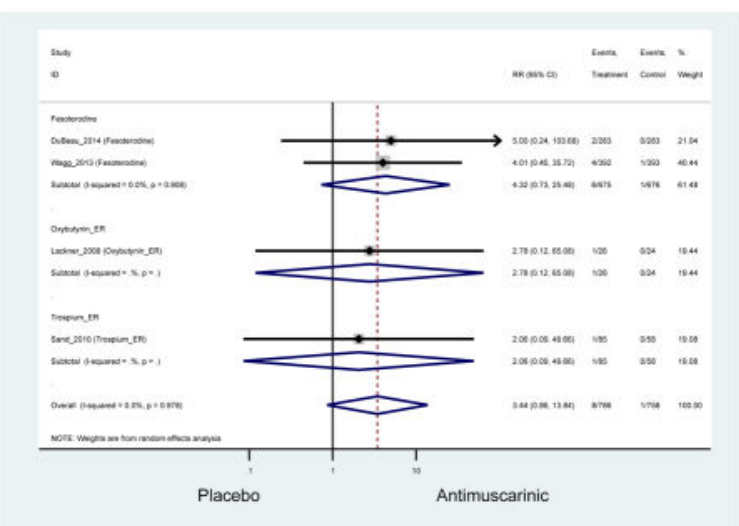

Appendix 9.150: Vertigo - Treatment Discontinuation (Antimuscarinics versus Placebo)

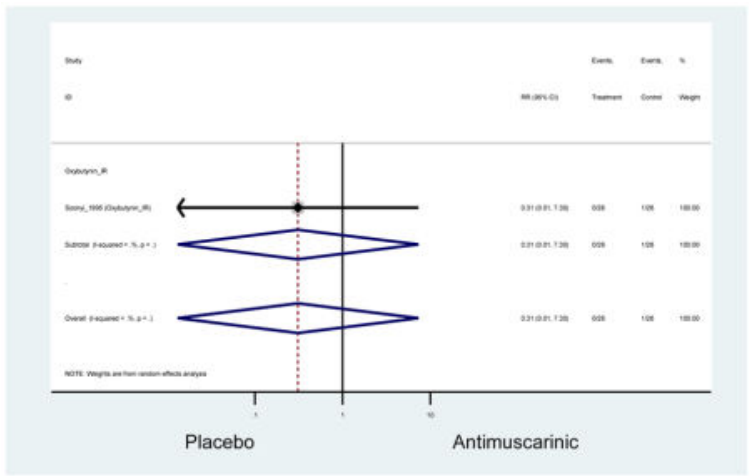

Appendix 9.151: Vision Blurred - Treatment Discontinuation (Antimuscarinics versus Placebo)

Zero Outcomes

Appendix 9.152: Withdrew Consent - Treatment Discontinuation (Antimuscarinics versus Placebo) 


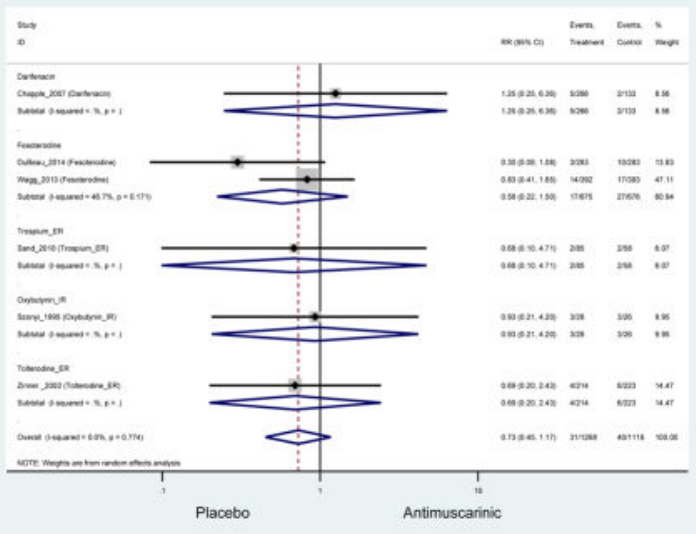

Appendix 9.153: Adverse Events - Treatment Discontinuation (Other Antimuscarinics versus Oxybutynin IR)

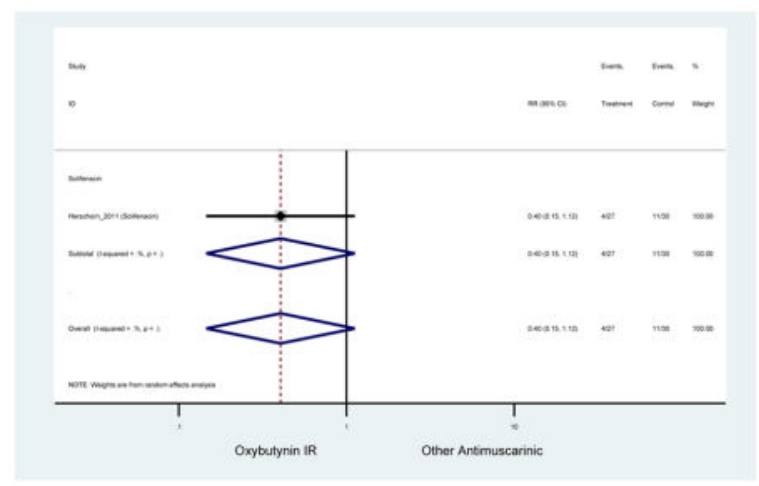

Appendix 9.154: Dry Mouth - Treatment Discontinuation (Other Antimuscarinics versus Oxybutynin IR)

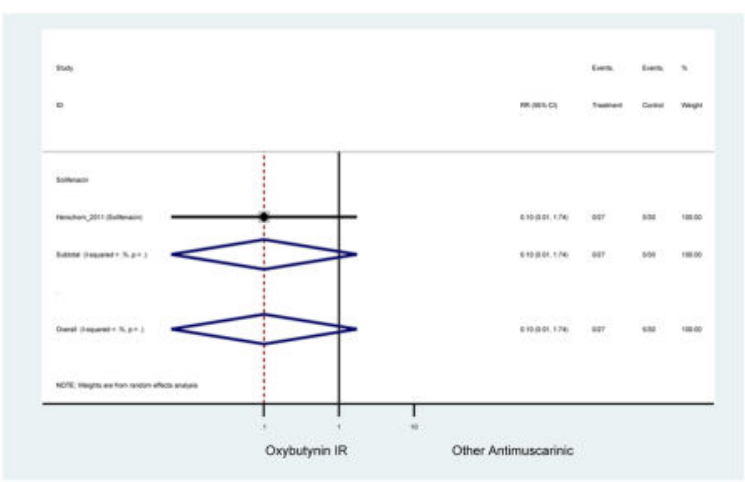

Appendix 9.155: Total Discontinuation - Treatment Discontinuation (Other Antimuscarinics versus Oxybutynin IR) 


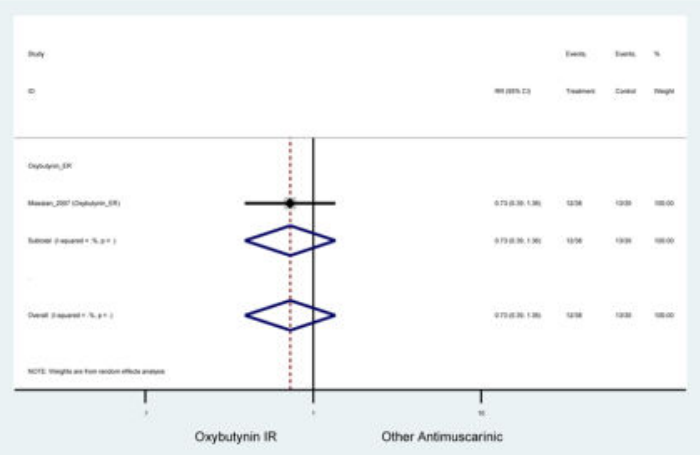

Appendix 9.156: Adverse Events - Treatment Discontinuation (Other Antimuscarinics versus Tolterodine IR)

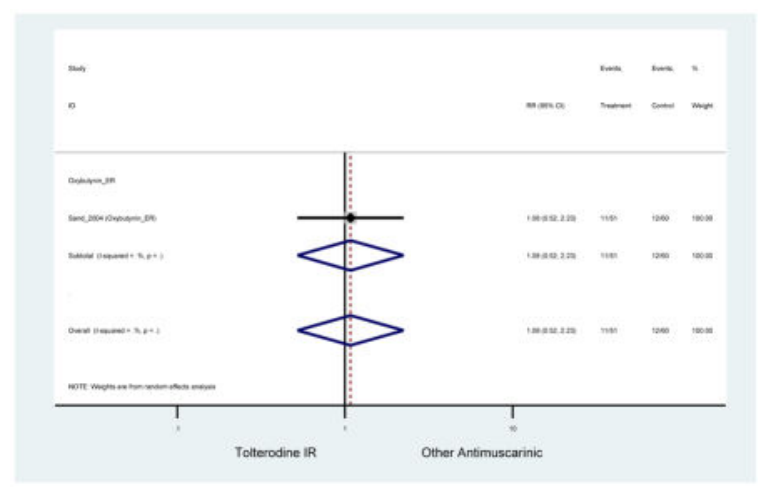

Appendix 9.157: Lost to Follow-up - Treatment Discontinuation (Other Antimuscarinics versus Tolterodine IR)

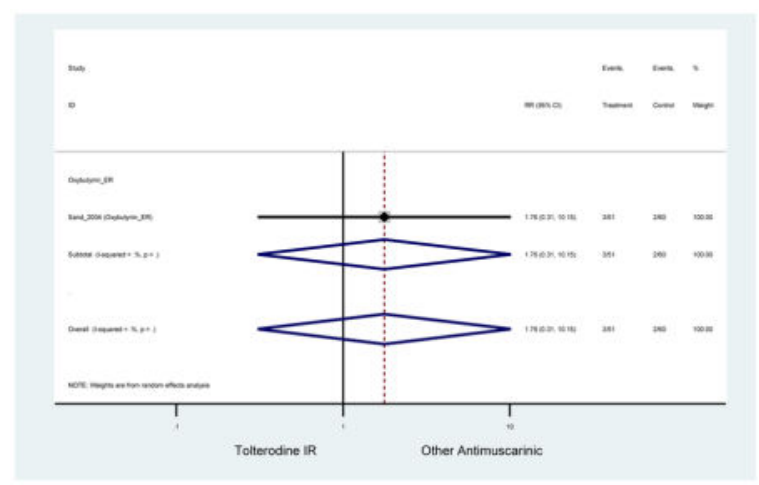

Appendix 9.158: Personal Reasons - Treatment Discontinuation (Other Antimuscarinics versus Tolterodine IR) 

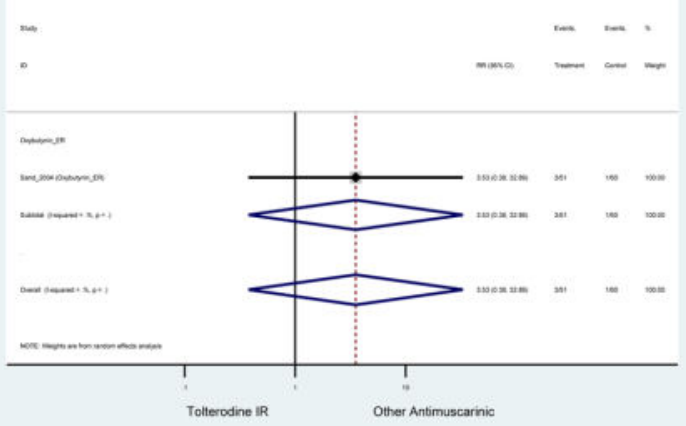

Appendix 9.159: Protocol Violation - Treatment Discontinuation (Other Antimuscarinics versus Tolterodine IR)

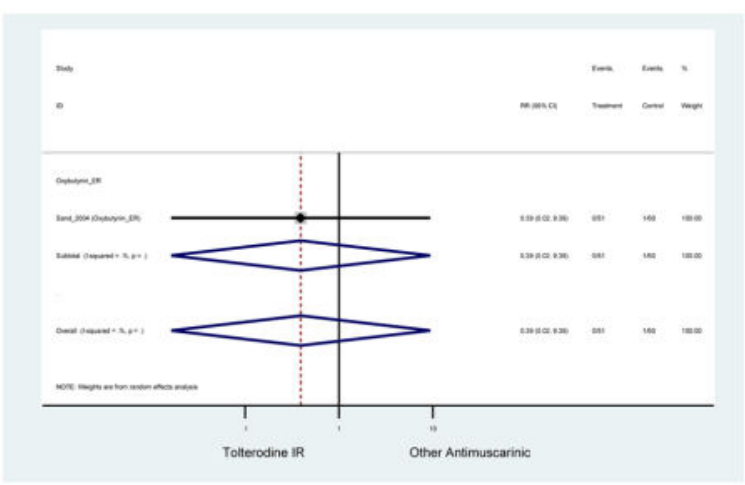

Appendix 9.160: Unsatisfactory Therapeutic effect - Treatment Discontinuation (Other Antimuscarinics versus Tolterodine IR)

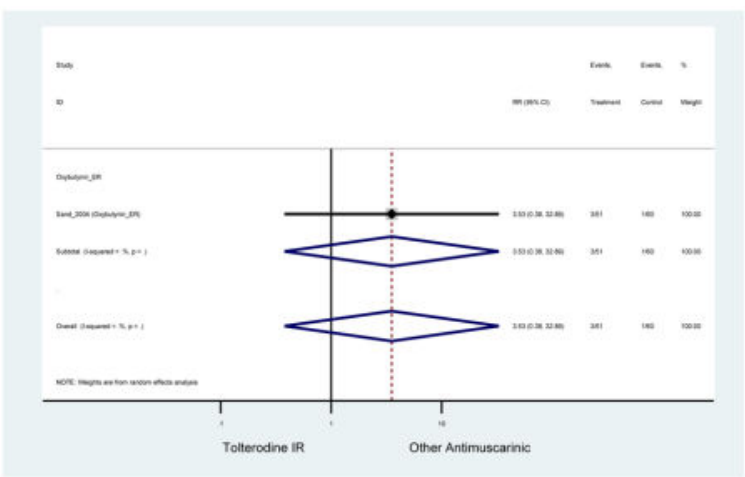

Appendix 9.161: Withdrew Consent - Treatment Discontinuation (Other Antimuscarinics versus Tolterodine IR) 

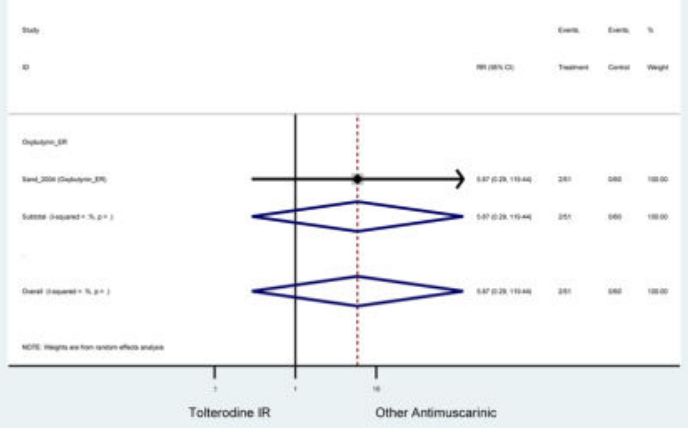

Appendix 9.162: Adverse Events - Treatment Discontinuation (Other Antimuscarinics versus Tolterodine ER)

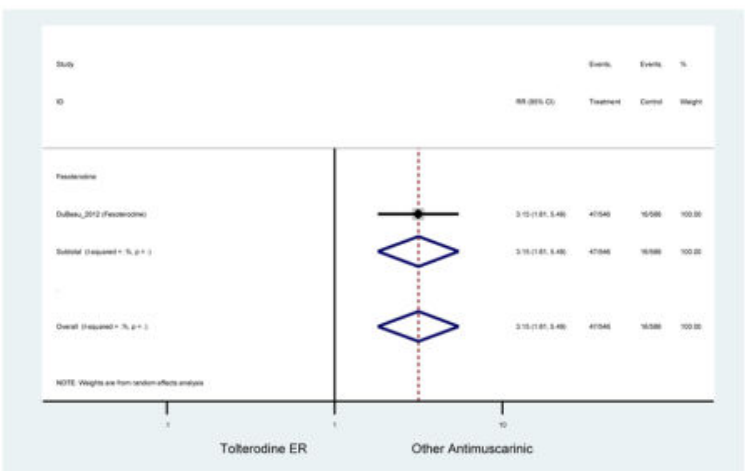

Appendix 9.163: Constipation - Treatment Discontinuation (Other Antimuscarinics versus Tolterodine ER)

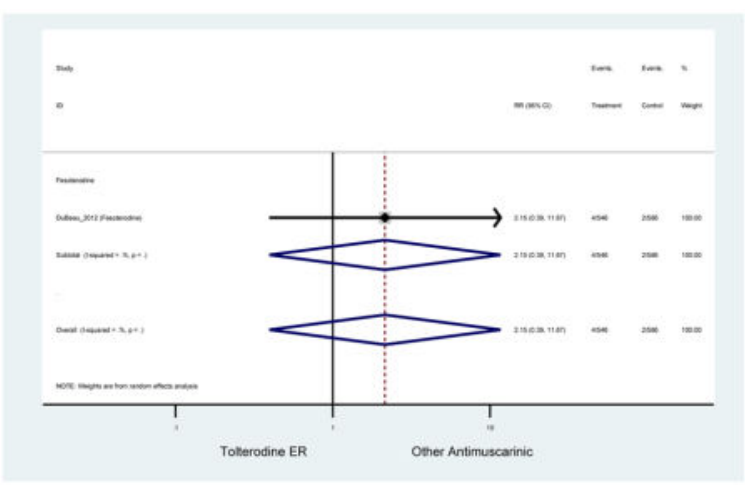

Appendix 9.164: Dry Mouth - Treatment Discontinuation (Other Antimuscarinics versus Tolterodine ER) 


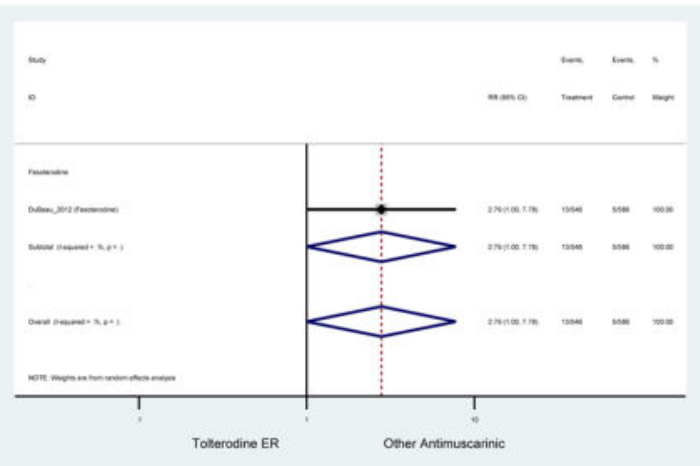

Appendix 9.165: Headache - Treatment Discontinuation (Other Antimuscarinics versus Tolterodine ER)

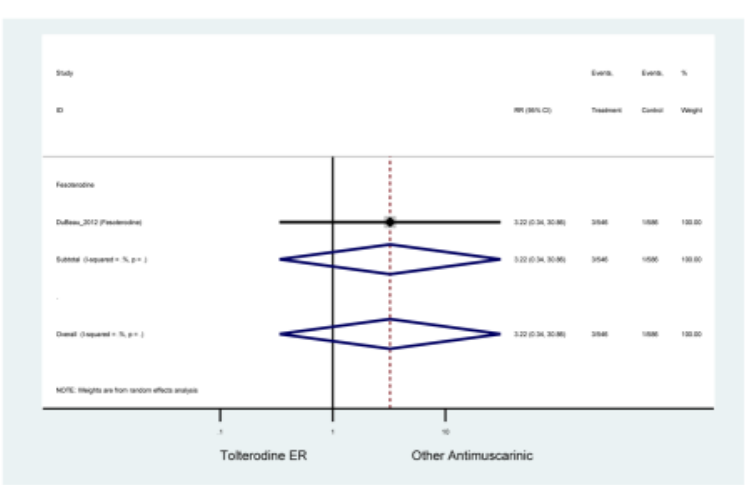

Appendix 9.166: Vision Blurred - Treatment Discontinuation (Other Antimuscarinics versus Tolterodine ER)

Zero Outcomes

Appendix 9.167: Gastrointestinal Combined Adverse Events (Antimuscarinic versus Placebo)

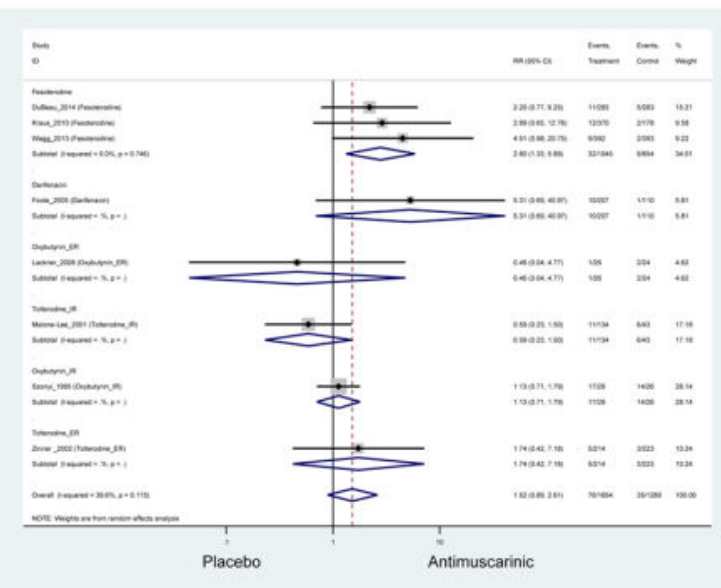

Arch Gerontol Geriatr. Author manuscript; available in PMC 2018 March 01. 
Appendix 9.168: Ocular/Visual Combined Adverse Events (Antimuscarinic versus Placebo)

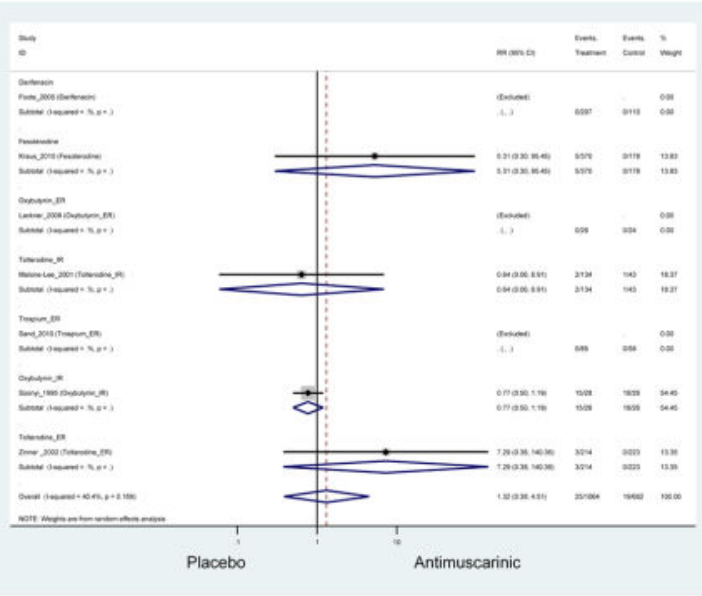

Appendix 9.169: Pain-related Combined Adverse Events (Antimuscarinic versus Placebo)

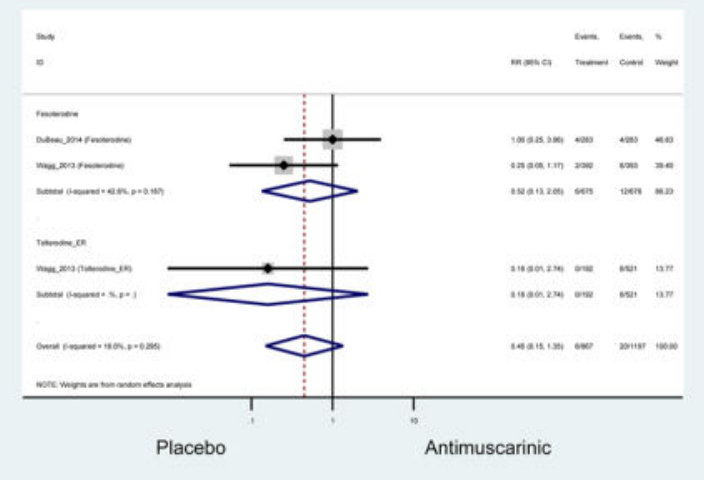

Appendix 9.170: Cardiac Combined Adverse Events (Antimuscarinic versus Placebo)

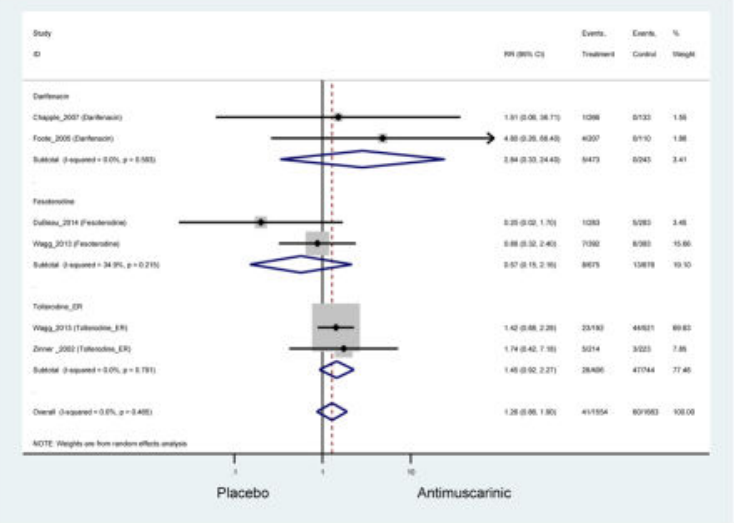

Appendix 9.171: Falls Combined Adverse Events (Antimuscarinic versus Placebo)

Arch Gerontol Geriatr. Author manuscript; available in PMC 2018 March 01. 


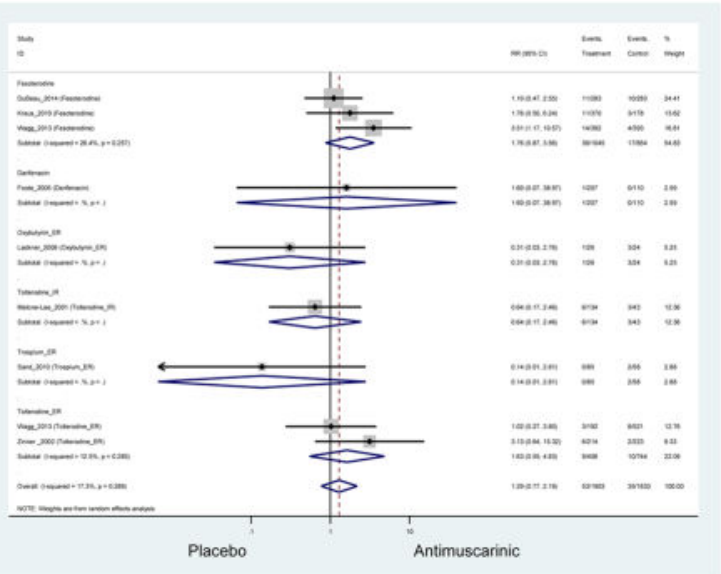

Appendix 9.172: Respiratory Tract-related Combined Adverse Events (Antimuscarinic versus Placebo)

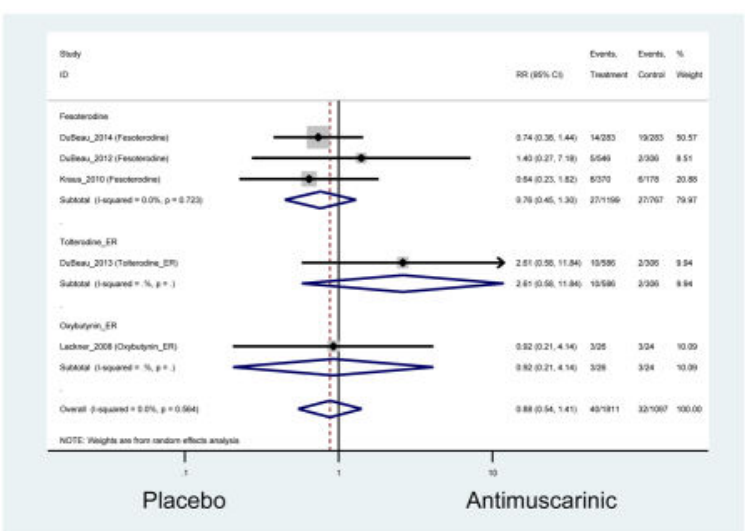

Appendix 9.173: Neurological/Cognitive Combined Adverse Events (Antimuscarinic versus Placebo)

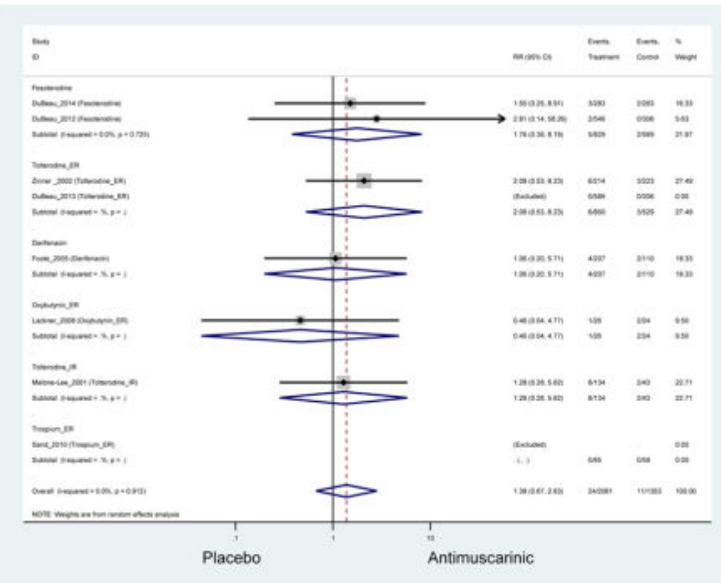

Appendix 9.174: Nasal Combined Adverse Events (Antimuscarinic versus Placebo)

Arch Gerontol Geriatr. Author manuscript; available in PMC 2018 March 01. 


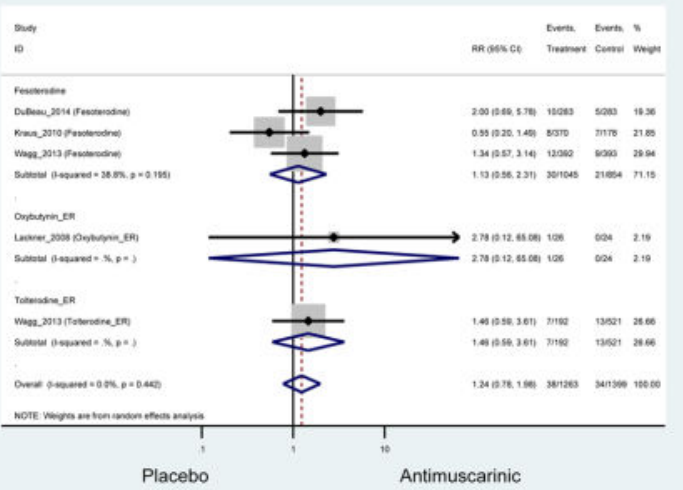

Appendix 9.175: Gastrointestinal Combined Adverse Events (Other Antimuscarinics versus Oxybutynin IR)

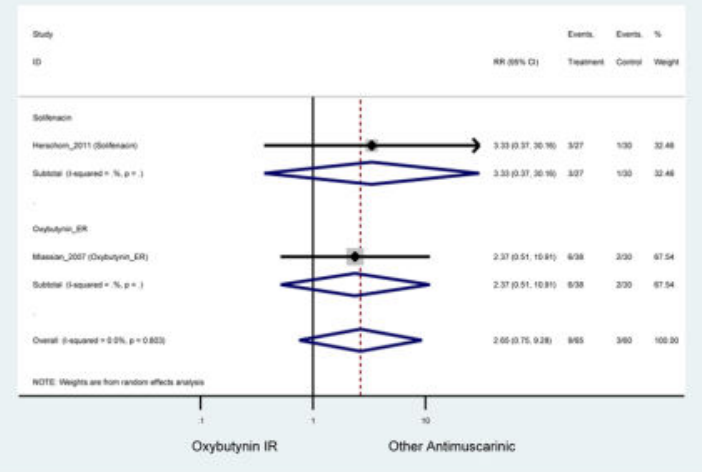

Appendix 9.176: Ocular/Visual Combined Adverse Events (Other Antimuscarinics versus Oxybutynin IR)

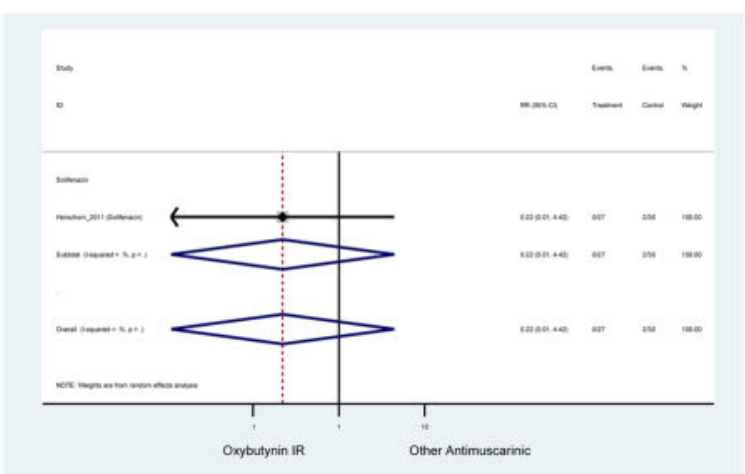

Appendix 9.177: Pain-related Combined Adverse Events (Other Antimuscarinics versus Oxybutynin IR)

Zero Outcomes 
Appendix 9.178: Falls Combined Adverse Events (Other Antimuscarinics versus Oxybutynin IR)

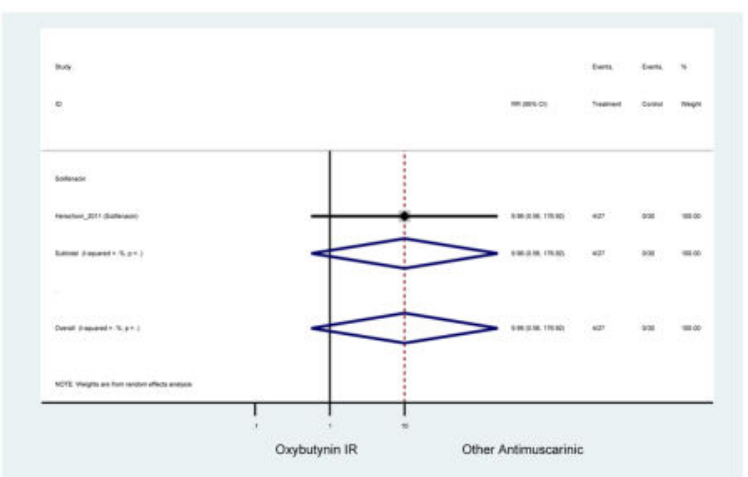

Appendix 9.179: Respiratory Tract-related Combined Adverse Events (Other Antimuscarinics versus Oxybutynin IR)

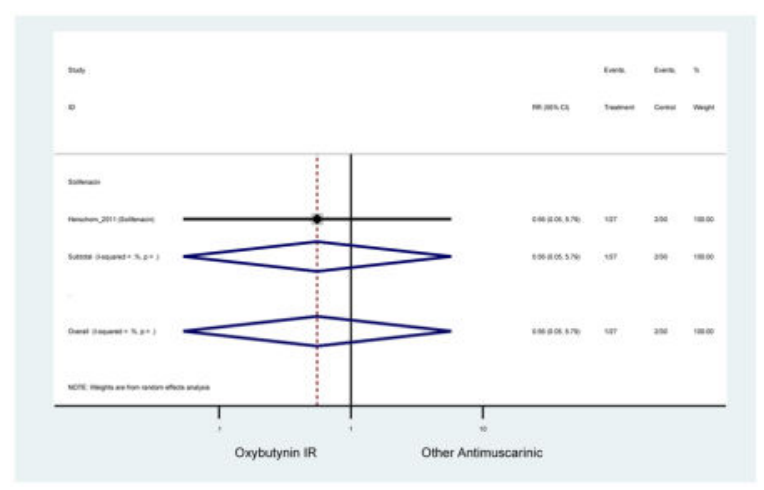

Appendix 9.180: Neurological/Cognitive Combined Adverse Events (Other Antimuscarinics versus Oxybutynin IR)

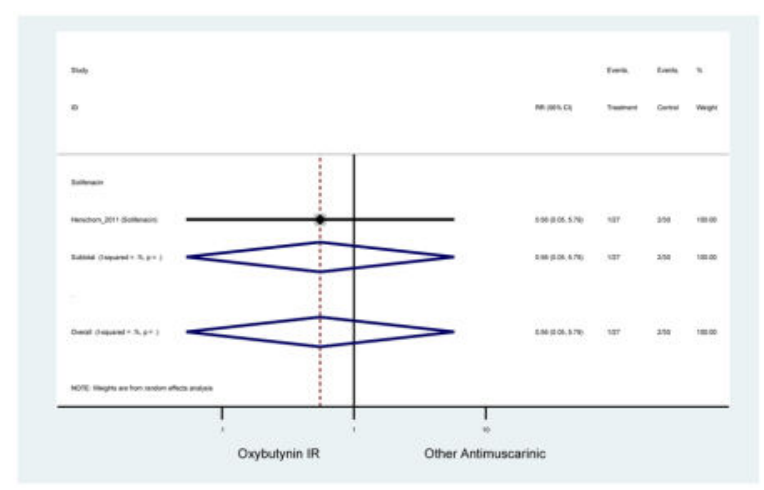

Appendix 9.181: Nasal Combined Adverse Events (Other Antimuscarinics versus Oxybutynin IR) 


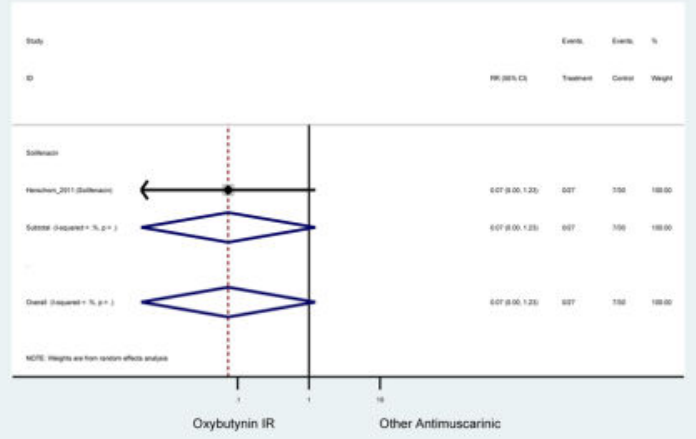

Appendix 9.182: Gastrointestinal Combined Adverse Events (Other Antimuscarinics versus Tolterodine IR)

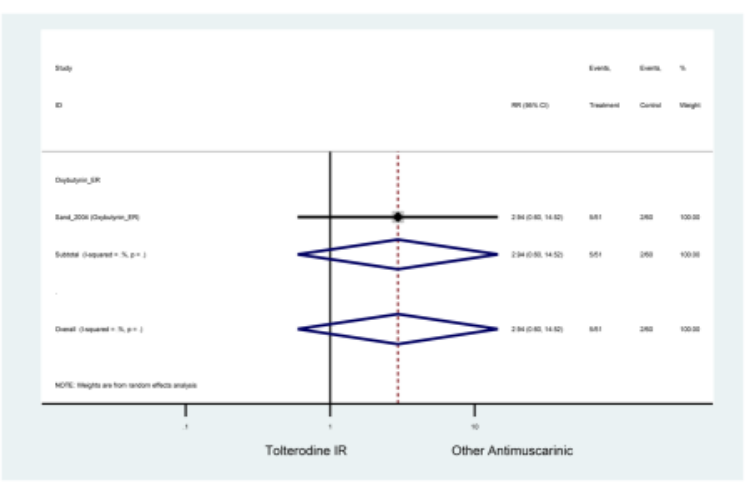

Appendix 9.183: Ocular/Visual Combined Adverse Events (Other Antimuscarinics versus Tolterodine IR)

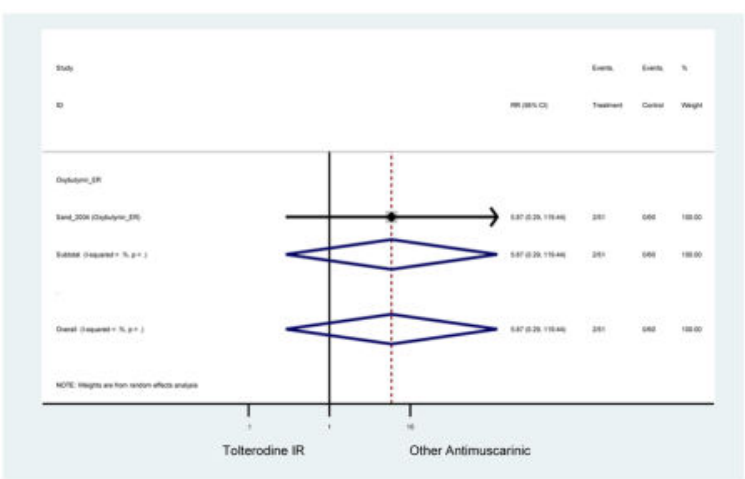

Appendix 9.184: Falls Combined Adverse Events (Other Antimuscarinics versus Tolterodine IR)

Zero Outcomes

Appendix 9.185: Neurological/Cognitive Combined Adverse Events (Other Antimuscarinics versus Tolterodine IR) 


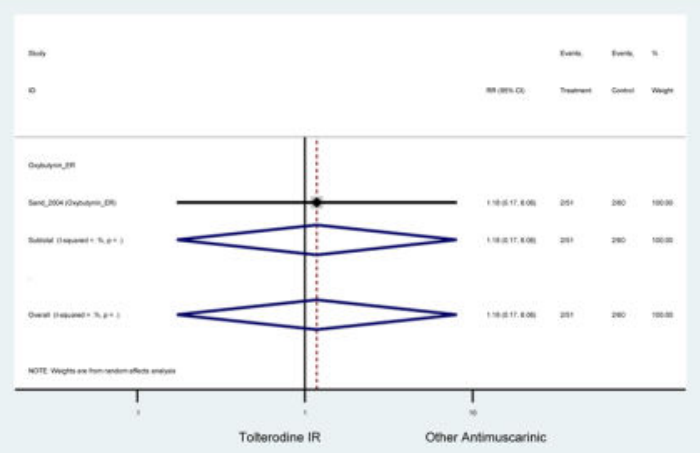

Appendix 9.186: Respiratory tract-related Combined Adverse Events (Other Antimuscarinics versus Tolterodine ER)

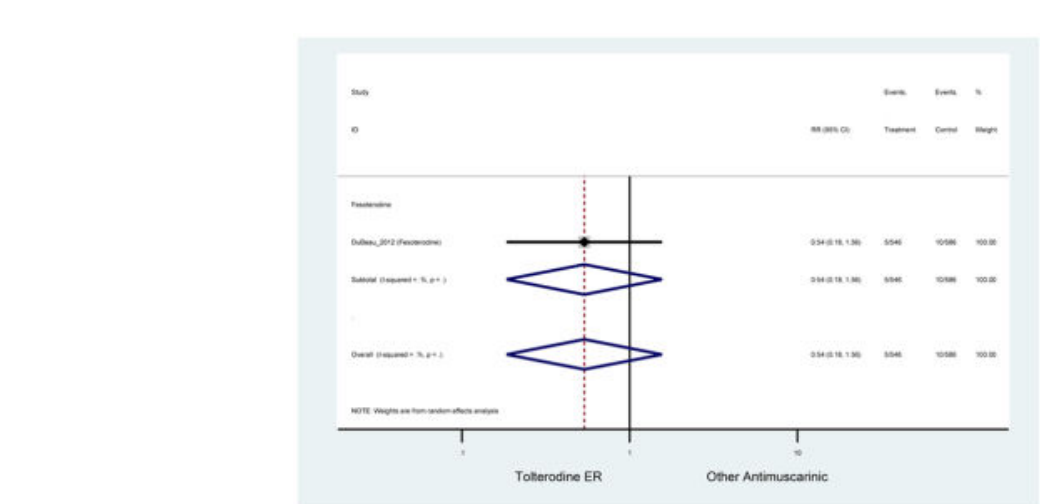




\section{Highlights}

- There are higher rates of anticholinergic AEs like dry mouth and constipation and treatment discontinuation in antimuscarinics versus placebo in the elderly

- There are higher rates of non-anticholinergic AEs, such as dyspepsia, dizziness, headache, and urinary tract infections in antimuscarinics versus placebo in the elderly

- In head-to-head trials, there are higher rates of AEs in fesoterodine compared to tolterodine extended-release in the elderly

- Due to the restrictive inclusion and exclusion criteria, these results should be further confirmed using pharmacoepidemiological techniques. 


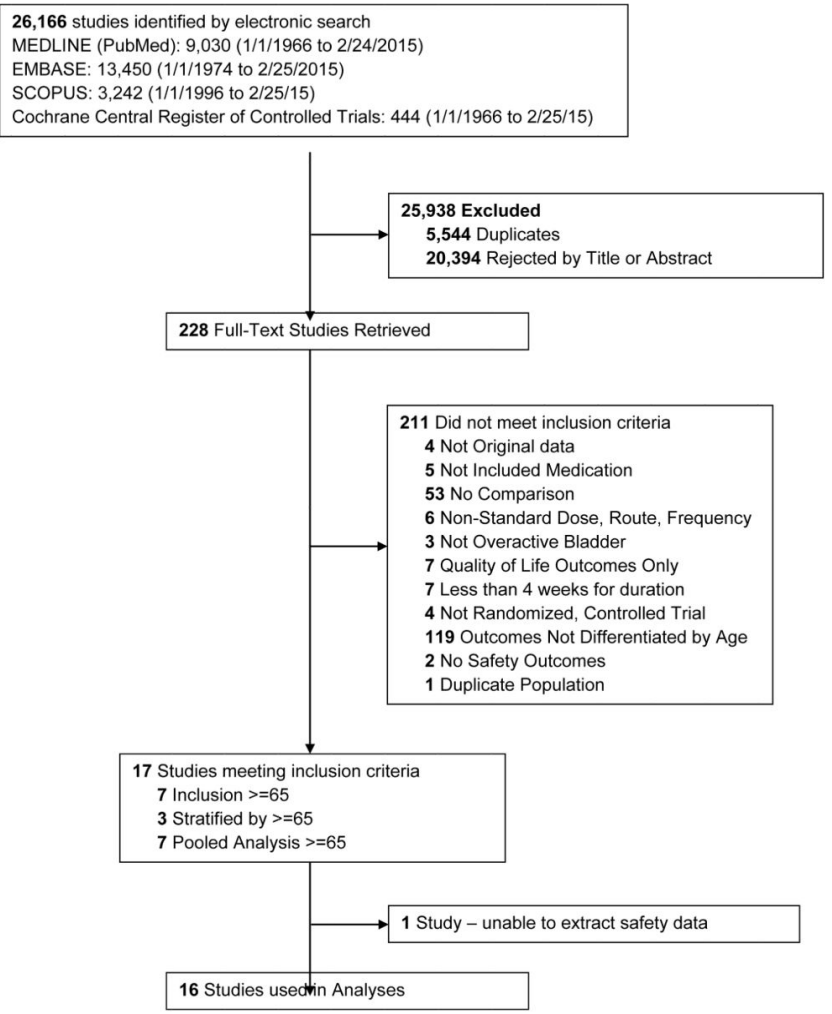

Figure 1.

Determination of studies 


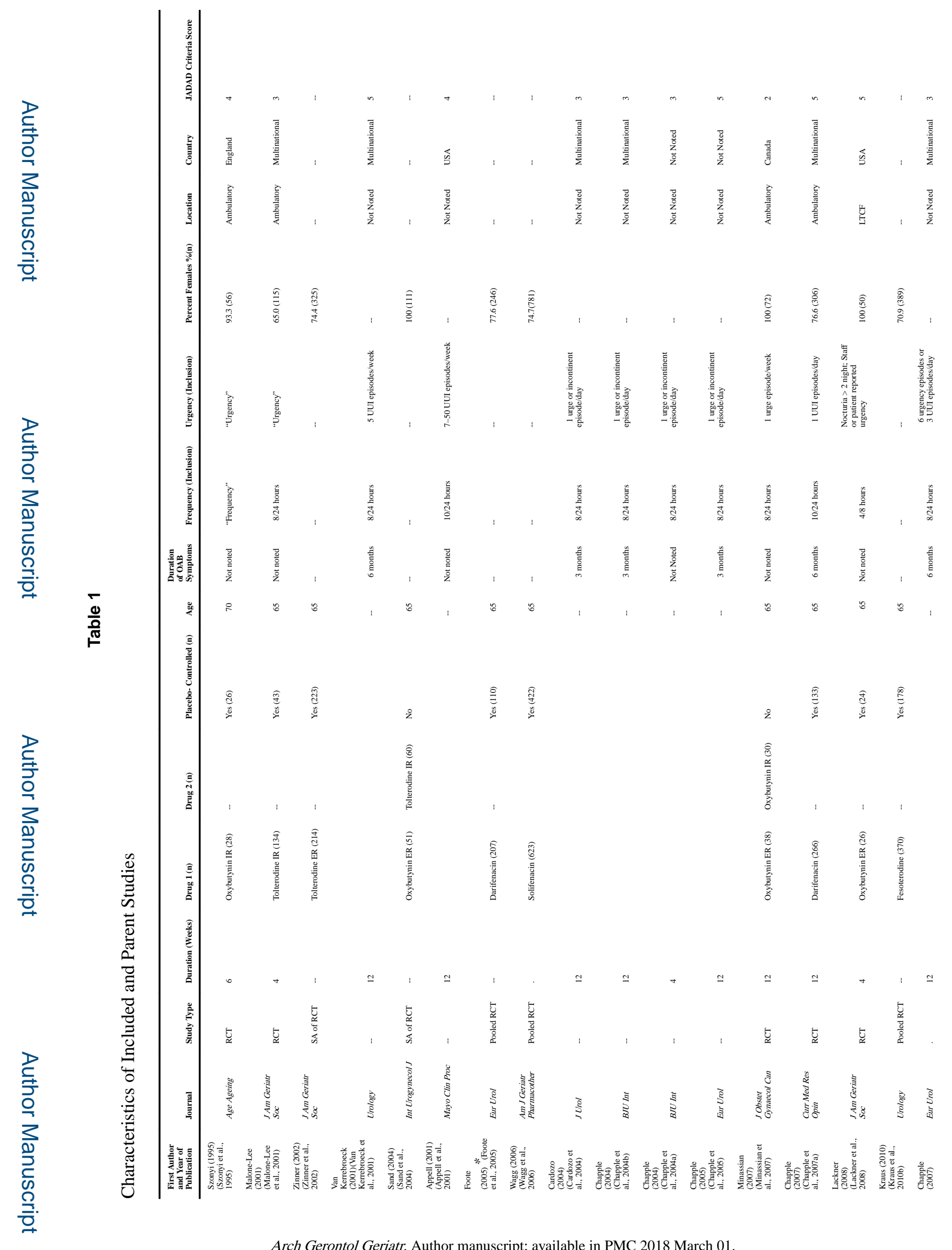

Arch Gerontol Geriatr. Author manuscript; available in PMC 2018 March 01. 


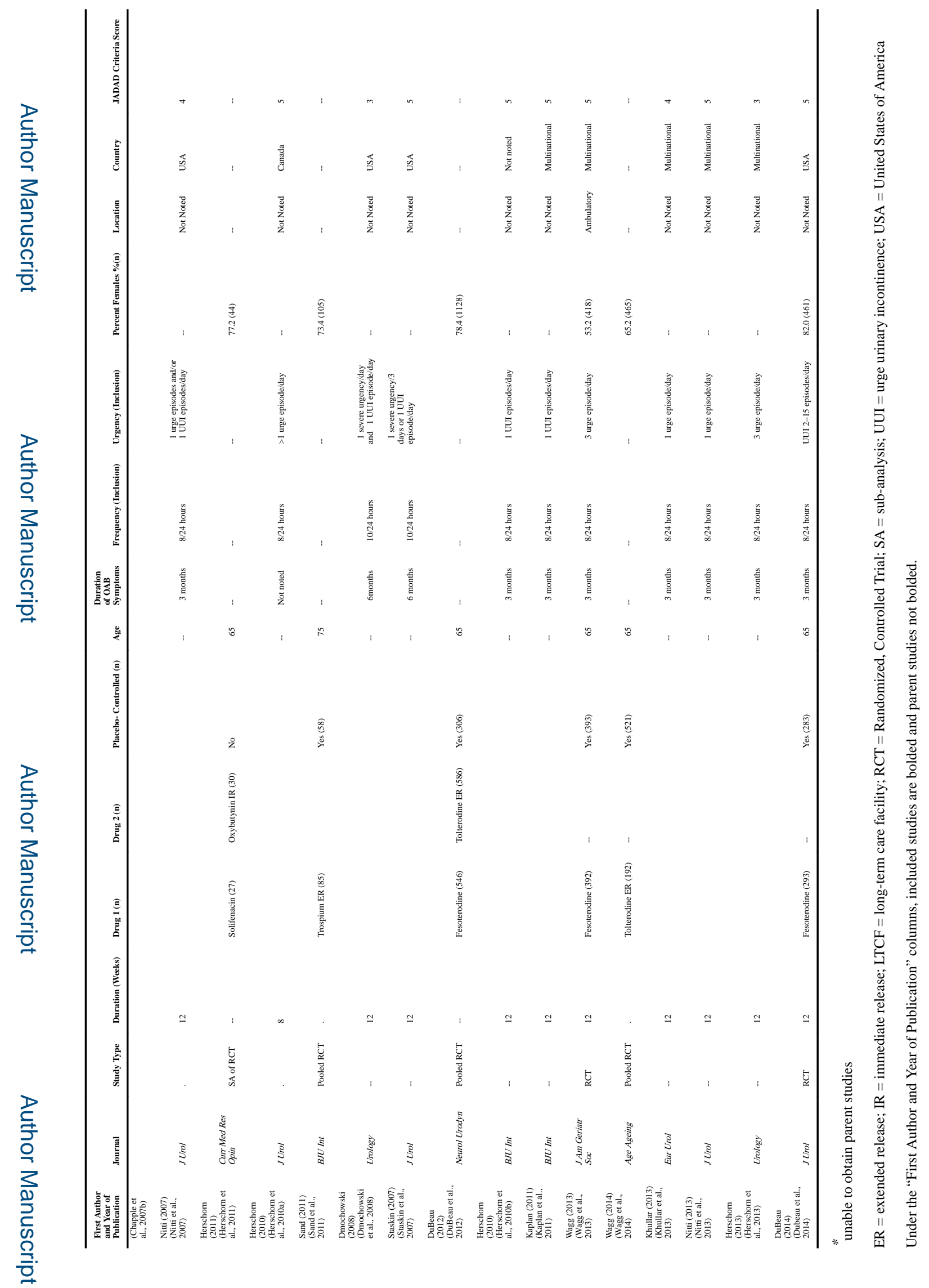

Arch Gerontol Geriatr. Author manuscript; available in PMC 2018 March 01. 


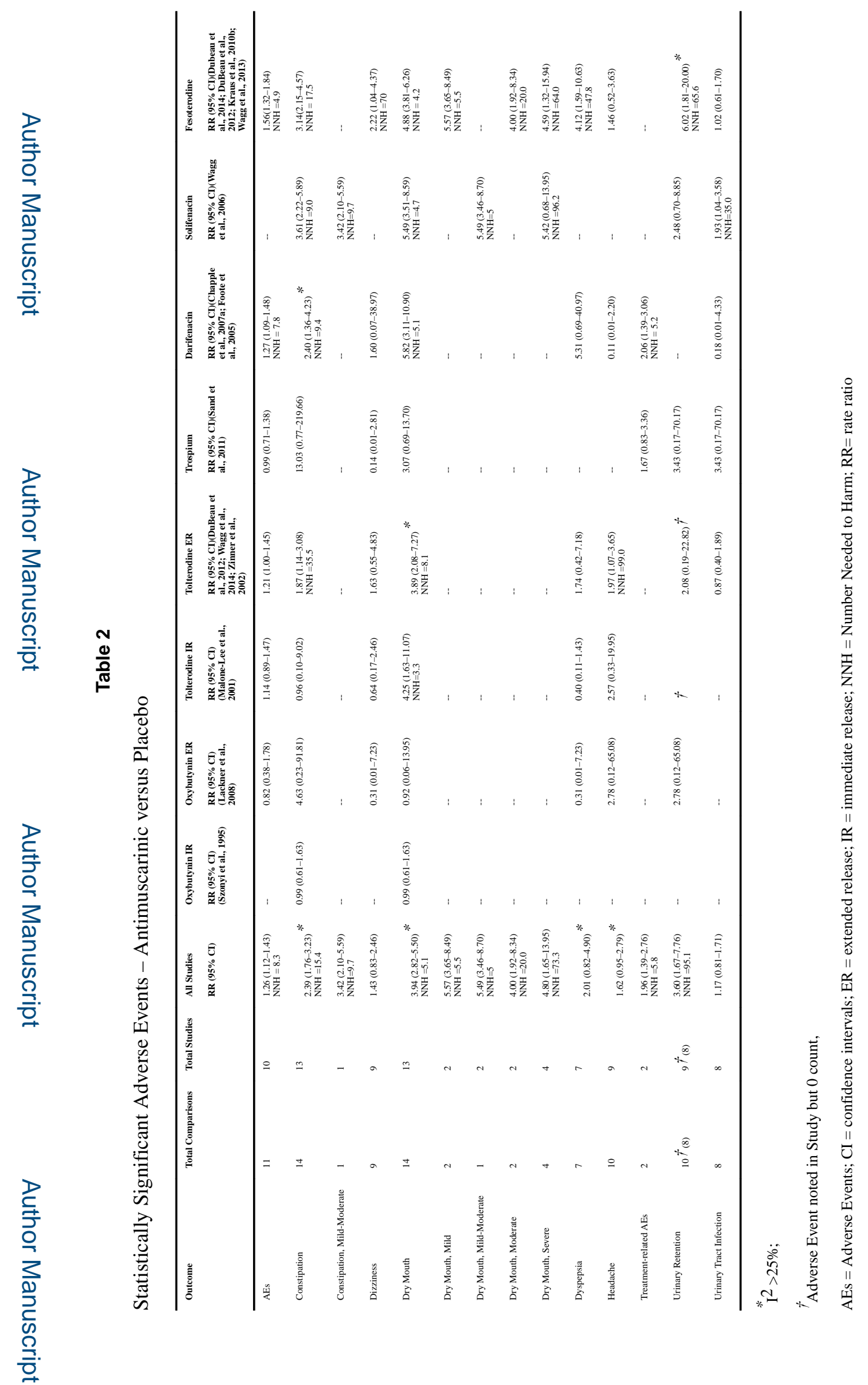

Arch Gerontol Geriatr. Author manuscript; available in PMC 2018 March 01. 


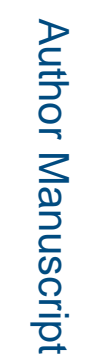

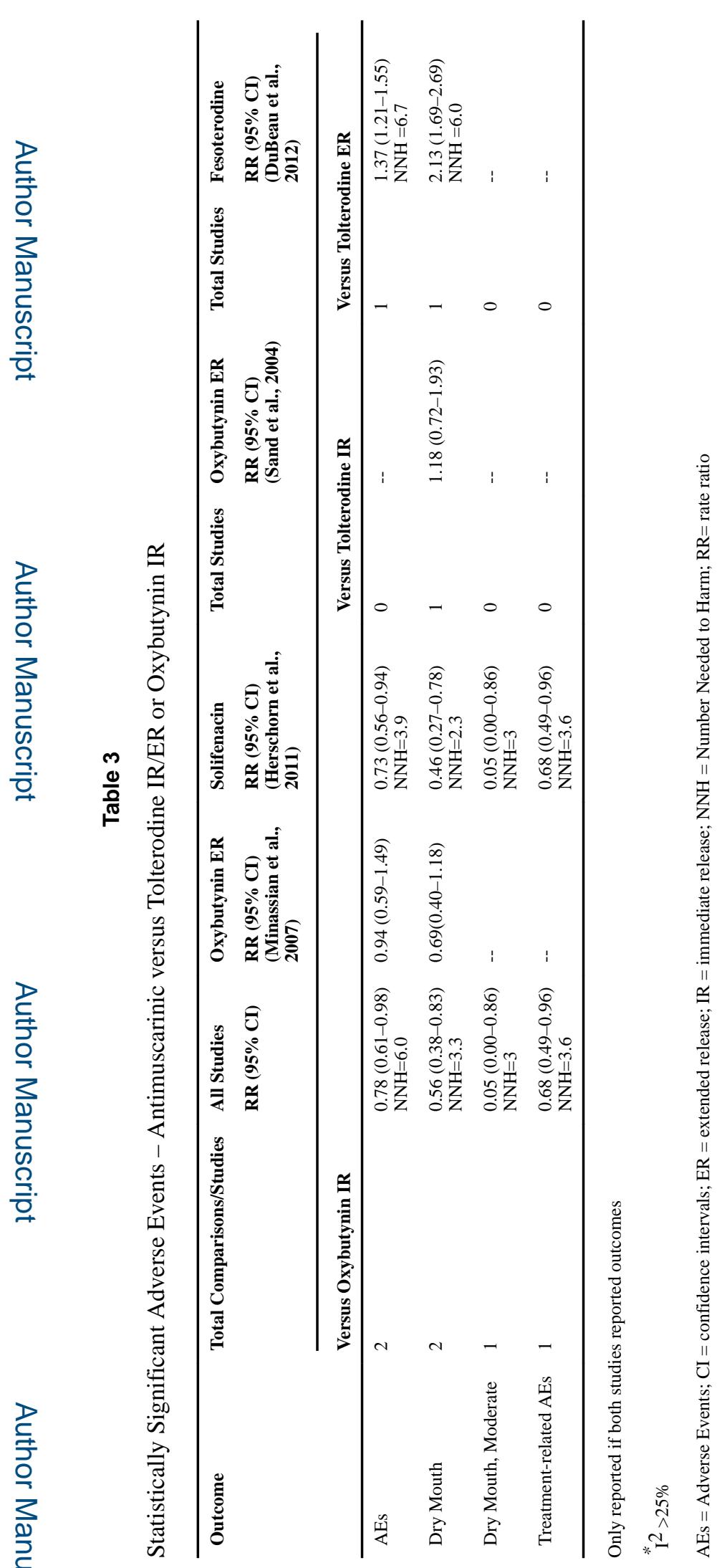

Arch Gerontol Geriatr. Author manuscript; available in PMC 2018 March 01. 


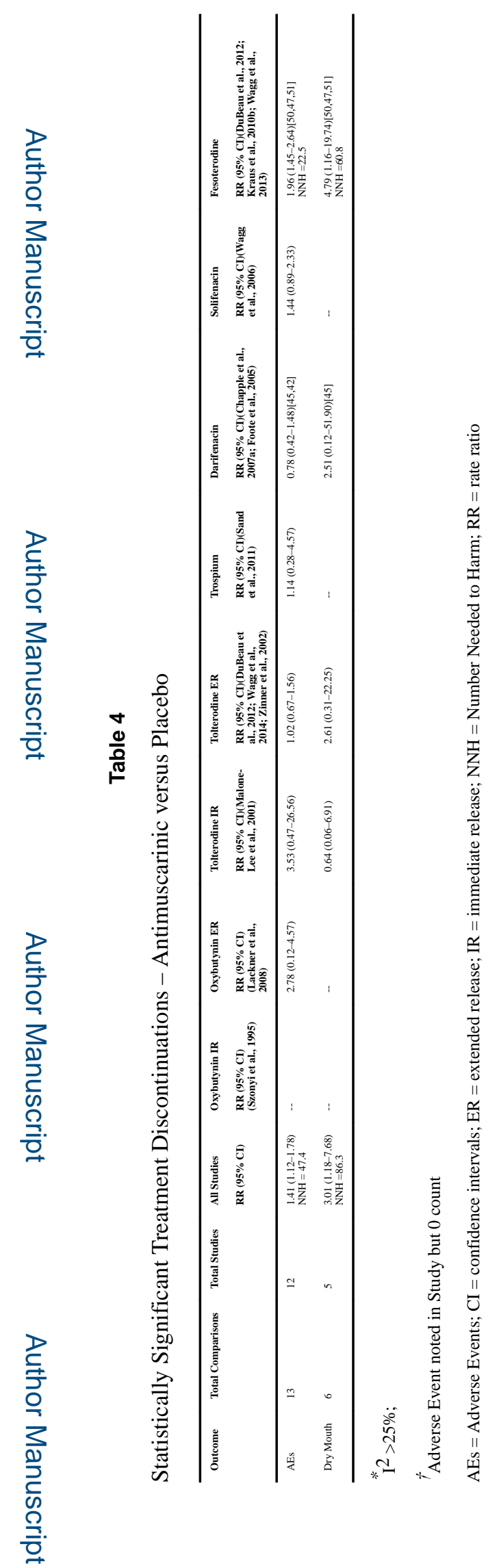

Arch Gerontol Geriatr. Author manuscript; available in PMC 2018 March 01. 


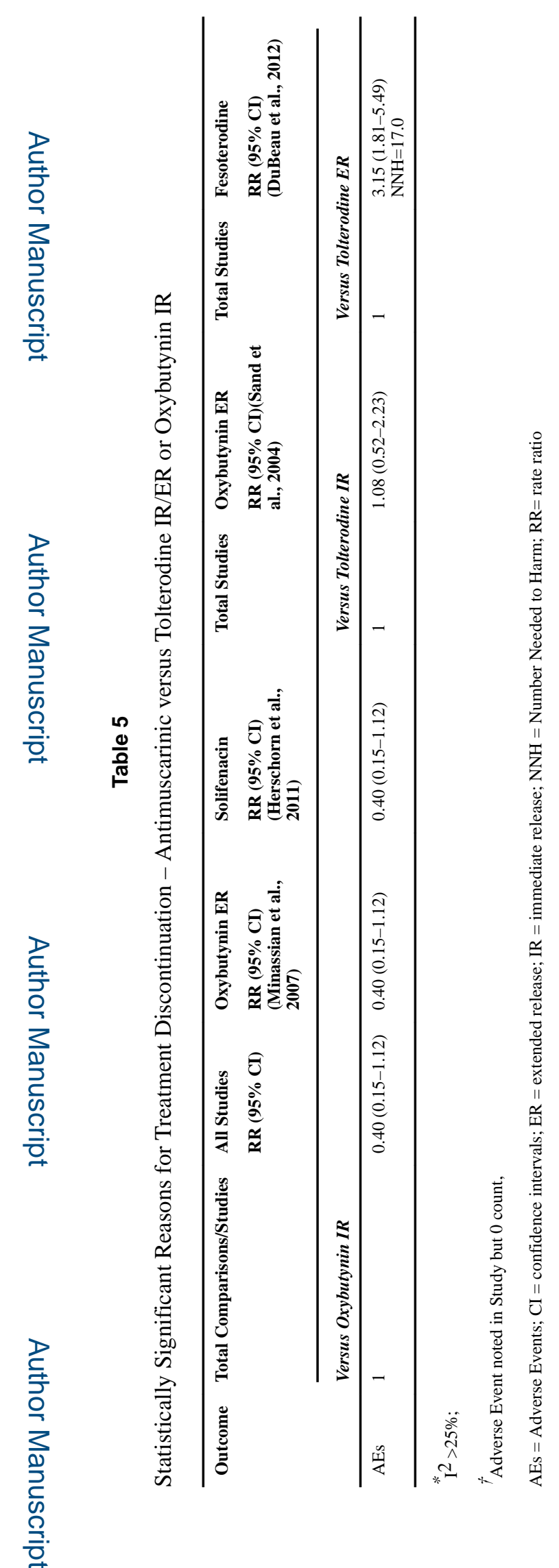

Arch Gerontol Geriatr. Author manuscript; available in PMC 2018 March 01. 